\title{
Superstability in simple finitary AECs
}

\author{
by \\ Tapani Hyttinen and Meeri Kesälä (Helsinki)
}

\begin{abstract}
We continue the study of finitary abstract elementary classes beyond $\aleph_{0^{-}}$ stability. We suggest a possible notion of superstability for simple finitary AECs, and derive from this notion several good properties for independence. We also study constructible models and the behaviour of Galois types and weak Lascar strong types in this context.

We show that superstability is implied by a-categoricity in a suitable cardinal. As an application we prove the following theorem: Assume that $(\mathbb{K}, \preccurlyeq \mathbb{K})$ is a simple, tame, finitary AEC, a-categorical in some cardinal $\kappa$ above the Hanf number such that $\operatorname{cf}(\kappa)>\omega$. Then $(\mathbb{K}, \preccurlyeq \mathbb{K})$ is a-categorical in each cardinal above the Hanf number.
\end{abstract}

\section{INTRODUCTION}

Saharon Shelah developed the context of abstract elementary classes as a platform to study classification theory for non-elementary classes. In this context one does not study structures in any specific language, but a class $\mathbb{K}$ of structures of the same similarity type with an abstract elementary substructure relation $\preccurlyeq \mathbb{K}$. This framework is very general, and one might need to refine the axioms of the class to generalize machinery from stability theory for AECs. Several different contexts have been studied, and most of them assume at least amalgamation (see [21], [22], [23], [25], [4], [7], [17] or [2]). We introduced the context of finitary abstract elementary classes. We assume amalgamation, joint embedding and arbitrarily large models in order to work inside a monster model. In addition we assume the LöwenheimSkolem number being countable and a property we call finite character. When $\mathscr{A}$ and $\mathscr{B}$ are models in the class $\mathbb{K}$, finite character says that we can detect whether $\mathscr{A} \preccurlyeq \mathbb{K} \mathscr{B}$ by only looking at finite tuples $\bar{a} \in \mathscr{A}$ and checking

2000 Mathematics Subject Classification: Primary 03C45; Secondary 03C52, 03 C95.

Key words and phrases: abstract elementary class, independence, superstability, primary model.

First author partially supported by the Academy of Finland, grant 1106753. Second author supported by the graduate school MALJA. 
whether the Galois type of the tuple $\bar{a}$ in $\mathscr{A}$ agrees with its Galois type in $\mathscr{B}$. The main non-elementary examples of finitary classes are homogeneous classes (see [18] or [14]), and excellent classes (see [20] or [16]). There are several frameworks of AECs with an abstract notion of independence, where the definition is not specified but only axioms for the independence calculus are given; see for example Shelah [24], Grossberg and Kolesnikov [5] or Grossberg and Lessmann [6]. See also Adler [1] for a first-order framework. Our work differs from these in that we construct the actual notion.

In the papers [11] and [12] we studied the $\aleph_{0^{-}}$stable case. (See also [10].) We introduced a notion of weak type and weak $\lambda$-stability for a cardinal $\lambda$. We also studied a notion of strong type called Lascar strong type, written Lstp, which is the equivalence class of a tuple in the finest invariant equivalence relation with a bounded number of equivalence classes. We defined a notion of independence with a built-in extension property in the style of [14]. We also found useful the concept of simplicity, which is the property that $\downarrow$ satisfies $\bar{a} \downarrow_{A} A$ for all tuples $\bar{a}$ and finite sets $A\left({ }^{1}\right)$. In the $\aleph_{0}$-stable case, simplicity guarantees that we have the independence calculus for all sets, not only for $\aleph_{0}$-saturated models. This approach generalizes the one in [13] for excellent classes.

The main point of interest in this paper is again a notion of independence. We find the obvious notion of superstability, namely weak stability in large enough cardinals, insufficient to gain good behaviour for the notion of independence. We call this notion weak superstability and take as the main notion the following.

Definition 1.1. We say that the class $(\mathbb{K}, \preccurlyeq \mathbb{K})$ is superstable if it is weakly stable in at least one cardinal and the following holds. Let $\left(A_{n}\right)_{n<\omega}$ be an increasing sequence of finite sets such that $\bigcup_{n<\omega} A_{n}$ is a model, and let $\bar{a}$ be a tuple. Then there is $n<\omega$ such that $\bar{a} \downarrow_{A_{n}} A_{n+1}$.

The properties of the notion of independence under superstability are collected in Theorems 3.5 and 3.13. In Theorem 3.5 we study a superstable simple finitary AEC. In Theorem 3.13 we also assume the TarskiVaught property and gain all the usual properties of non-forking of complete types. The Tarski-Vaught property makes it possible to have countable constructible models. It says that we have countably many "abstract formulas" such that each set which is "existentially closed" relative to them is a $\mathbb{K}$-elementary substructure of the monster model. We also prove that $\aleph_{0}$-stable simple finitary classes are superstable (Corollary 3.28 ) and have the Tarski-Vaught property (Remark 3.9).

$\left({ }^{1}\right)$ In [11] and [12] we actually studied an a priori stronger notion but we will see that the notions agree under $\aleph_{0}$-stability. 
The most important notion of type in the context of AEC is Galois type. The notion was introduced by Shelah, and named Galois type by Grossberg in [4]. In our context two tuples $\bar{a}$ and $\bar{b}$ have the same Galois type over a set $A$, written $\operatorname{tp}^{\mathrm{g}}(\bar{a} / A)=\operatorname{tp}^{\mathrm{g}}(\bar{b} / A)$, if there is an automorphism of the monster model mapping $\bar{a}$ to $\bar{b}$ and fixing $A$ pointwise. The behaviour of these types is a key question in model theory.

Grossberg and VanDieren have studied abstract elementary classes with amalgamation and $\mu$-tameness for some $\mu$ (see [7]-[9]). The class $(\mathbb{K}, \preccurlyeq \mathbb{K})$ is said to be $\mu$-tame if for any tuples $\bar{a}$ and $\bar{b}$ and a model $\mathscr{A}, \operatorname{tp}^{\mathrm{g}}(\bar{a} / \mathscr{A}) \neq$ $\operatorname{tp}^{\mathrm{g}}(\bar{a} / \mathscr{A})$ implies that there is a submodel $\mathscr{A}_{0} \preccurlyeq \mathbb{K} \mathscr{A}$ such that $\left|\mathscr{A}_{0}\right| \leq \mu$ and $\operatorname{tp}^{\mathrm{g}}\left(\bar{a} / \mathscr{A}_{0}\right) \neq \operatorname{tp}^{\mathrm{g}}\left(\bar{a} / \mathscr{A}_{0}\right)$. This assumption implies many good properties for an abstract elementary class, for example we gain upwards categoricity transfer from a successor cardinal $\kappa^{+}>\max \left\{\operatorname{LS}(\mathbb{K})^{+}, \mu\right\}$. However, in many examples Galois types have finite character, that is, if the Galois types of $\bar{a}$ and $\bar{b}$ differ over a set $A$, there is some finite subset $A_{0} \subset A$ such that their types differ already over $A_{0}$. Elementary classes as well as homogeneous classes have this property. Also in excellent classes the same holds when $A$ is assumed to be a model, and in $\aleph_{0}$-stable finitary classes when $A$ is a countable model.

We take as our basic notion of type the weak Lascar strong type, which has finite character by definition. Two tuples $\bar{a}$ and $\bar{b}$ have the same weak Lascar strong type over $A$, written $\operatorname{Lstp}^{\mathrm{w}}(\bar{a} / A)=\operatorname{Lstp}^{\mathrm{w}}(\bar{b} / A)$, if for all finite $A_{0} \subset A$ we have $\operatorname{Lstp}\left(\bar{a} / A_{0}\right)=\operatorname{Lstp}\left(\bar{b} / A_{0}\right)$. We study the relation between these types and Galois types in simple finitary classes. Assuming superstability and the Tarski-Vaught property we deduce that when $A$ is a countable set and $\operatorname{tp}^{\mathrm{g}}(\bar{a} / A) \neq \operatorname{tp}^{\mathrm{g}}(\bar{b} / A)$, there is a finite $A_{0} \subset A$ such that $\operatorname{Lstp}\left(\bar{a} / A_{0}\right) \neq \operatorname{Lstp}\left(\bar{b} / A_{0}\right)$ (Theorem 3.19). If we also assume $\aleph_{0}$-tameness, the same holds when $A$ is an arbitrary model (Theorem 3.20), and furthermore if $A$ is an a-saturated model, we find a finite $A_{0} \subset A$ such that $\operatorname{tp}^{\mathrm{g}}\left(\bar{a} / A_{0}\right) \neq \operatorname{tp}^{\mathrm{g}}\left(\bar{b} / A_{0}\right)$ (Theorem 3.21). A model $\mathscr{A}$ is a-saturated if every Lascar strong type over a finite subset is realized in $\mathscr{A}$.

In the $\aleph_{0}$-stable case, the class of $\aleph_{0}$-saturated models of $\mathbb{K}$, written $\mathbb{K}_{\omega}$, is an interesting subclass of $\mathbb{K}$. Splitting behaves well in this class and we have the full categoricity transfer in $\left(\mathbb{K}_{\omega}, \preccurlyeq \mathbb{K}\right)$, when $(\mathbb{K}, \preccurlyeq \mathbb{K})$ is an $\aleph_{0}$-stable and $\aleph_{0}$-tame simple finitary class (Corollary 4.14(1) of [12]). In this paper we study the class $\left(\mathbb{K}_{\mathrm{a}}, \preccurlyeq \mathbb{K}\right)$, where $\mathbb{K}_{\mathrm{a}}$ is the class of a-saturated models of $\mathbb{K}$. Note that when $(\mathbb{K}, \preccurlyeq \mathbb{K})$ is a finitary class, the class $\left(\mathbb{K}_{\mathrm{a}}, \preccurlyeq \mathbb{K}\right)$ is an abstract elementary class but not necessarily finitary, since its LöwenheimSkolem number might be uncountable. We define a-categoricity to mean categoricity for the class $\left(\mathbb{K}_{\mathrm{a}}, \preccurlyeq \mathbb{K}\right)$, and show that a-categoricity in certain cardinals implies superstability for $\left(\mathbb{K}, \preccurlyeq_{\mathbb{K}}\right)$. In Section 4 we define an isolation notion for weak Lascar strong type and a concept of an a-primary 
model. We prove an a-categoricity transfer result and state some open questions.

After this paper was submitted, David Kueker announced new results that are very relevant to our framework [15]. His results state that any finitary class is closed under $L_{\infty \omega}$-equivalence, $\preccurlyeq_{\mathbb{K}}$ coincides with the notion of $L_{\infty \omega}$-elementary substructure over $\aleph_{0}$-saturated models and weak types equal $L_{\infty \omega}$-types in the monster model. Furthermore, if a finitary class is $\aleph_{0}$-stable, the class $\left(\mathbb{K}_{\omega}, \preccurlyeq \mathbb{K}\right)$ is definable with a complete sentence in $L_{\omega_{1} \omega}$, and $L_{\infty \omega}$ can be replaced with a countable fragment of $L_{\omega_{1} \omega}$. We decided not to change the notation in this paper, although some notions like "abstract formula" can now be seen in a different light. However, we decided to rewrite the paper [12] taking these results into account. The paper will include discussion on how our work fits into the study of non-elementary, syntactically defined classes and will present several examples. The examples also show that there are simple, superstable finitary classes with the Tarski-Vaught property which are not definable with a sentence in $L_{\omega_{1} \omega}$. We will also give a proof that the example studied in [3] is simple, and hence there is a simple, finitary, $\aleph_{0}$-stable class which is not tame and thus neither homogeneous nor excellent.

We assume the reader to be familiar with the notions of abstract elementary classes and the most common concepts in stability theory for these, like amalgamation, Galois type and the monster model. We also refer to the results in [11] and [12] without proof.

\section{INDEPENDENCE}

In [12] we studied finitary AECs $(\mathbb{K}, \preccurlyeq \mathbb{K})$, which are abstract elementary classes with Löwenheim-Skolem number $\aleph_{0}$, amalgamation, joint embedding, arbitrarily large models and finite character. Models in these classes are models of a countable vocabulary $\tau$. First we defined a notion of a Galois type over the empty set for a tuple $\bar{a}$ in a model $\mathscr{A}$, written $\operatorname{tp}^{\mathrm{g}}(\bar{a} / \emptyset, \mathscr{A})$, such that

$$
\operatorname{tp}^{\mathrm{g}}(\bar{a} / \emptyset, \mathscr{A})=\operatorname{tp}^{\mathrm{g}}(\bar{b} / \emptyset, \mathscr{B})
$$

if there is $\mathscr{C} \in \mathbb{K}$ and $\mathbb{K}$-embeddings $f: \mathscr{A} \rightarrow \mathscr{C}$ and $g: \mathscr{B} \rightarrow \mathscr{C}$ such that $f(\bar{a})=g(\bar{b})$. Then we defined finite character to be the following property.

Assumption 2.1 (Finite character). Assume that $\mathscr{A} \subset \mathscr{B}$ are models and for all tuples $\bar{a} \in \mathscr{A}$,

$$
\operatorname{tp}^{\mathrm{g}}(\bar{a} / \emptyset, \mathscr{A})=\operatorname{tp}^{\mathrm{g}}(\bar{a} / \emptyset, \mathscr{B}) .
$$

Then $\mathscr{A}$ is a $\mathbb{K}$-elementary submodel of $\mathscr{B}$.

A useful consequence of the finite character property is that, when $\mathscr{A} \preccurlyeq \mathbb{K} \mathscr{B}$ and $f: \mathscr{A} \rightarrow \mathscr{B}$ is an embedding, then $f$ is a $\mathbb{K}$-elementary 
embedding if and only if $f$ preserves the Galois types of finite tuples, i.e.

$$
\operatorname{tp}^{\mathrm{g}}(\bar{a} / \emptyset, \mathscr{B})=\operatorname{tp}^{\mathrm{g}}(f(\bar{a}) / \emptyset, \mathscr{B})
$$

for all tuples $\bar{a} \in \mathscr{A}$. With the usual Jónsson-Fraïssé construction we obtain the following theorem.

TheOREM 2.2 (Monster model). Let $\mu$ be a cardinal. There is $\mathfrak{M} \in \mathbb{K}$ such that:

(1) Universality: For all $\mathscr{A} \in \mathbb{K}$ such that $|\mathscr{A}|<\mu$, there is a $\mathbb{K}$ embedding $f: \mathscr{A} \rightarrow \mathfrak{M}$.

(2) $\mathbb{K}$-homogeneity: For all $\mathscr{A} \preccurlyeq \mathbb{K} \mathfrak{M}$ such that $|\mathscr{A}|<\mu$ and $\mathbb{K}$-elementary $f: \mathscr{A} \rightarrow \mathfrak{M}$, there is $g \in \operatorname{Aut}(\mathfrak{M})$ extending $f$.

We say that a set $A \subset \mathfrak{M}$ is $\mathfrak{M}$-bounded if $|A|<\mu$.

We will always assume that all sets we consider are contained in a monster model $\mathfrak{M}$, and are $\mathfrak{M}$-bounded. We say that $\mathscr{A}$ is a model if $\mathscr{A} \in \mathbb{K}$ and $\mathscr{A} \preccurlyeq \mathbb{K} \mathfrak{M}$.

We will only consider such monster models when $\mu$ is a limit cardinal. With finite character we get an even stronger version of (2), namely:

$\left(2^{\prime}\right)$ For all $\mathscr{A} \preccurlyeq \mathbb{K} \mathfrak{M}$ such that $|\mathscr{A}|<\mu$ and mappings $f: \mathscr{A} \rightarrow \mathfrak{M}$ such that for all finite tuples $\bar{a} \in \mathscr{A}$,

$$
\operatorname{tp}^{\mathrm{g}}(\bar{a} / \emptyset, \mathfrak{M})=\operatorname{tp}^{\mathrm{g}}(f(\bar{a}) / \emptyset, \mathfrak{M}),
$$

there is $g \in \operatorname{Aut}(\mathfrak{M})$ extending $f$.

The monster models are $\mu$-saturated in the following sense: if $\mathfrak{M} \preccurlyeq \mathbb{K} \mathfrak{M}^{\prime}$ are two monster models and $A \subset \mathfrak{M}, B \subset \mathfrak{M}^{\prime}$ are $\mathfrak{M}$-bounded sets, there is an automorphism of $\mathfrak{M}^{\prime}$ fixing $A$ and mapping $B$ into $\mathfrak{M}$.

When $\bar{a}$ and $\bar{b}$ are in a monster model $\mathfrak{M}$, we have $\operatorname{tp}^{\mathrm{g}}(\bar{a} / \emptyset, \mathfrak{M})=$ $\operatorname{tp}^{\mathrm{g}}(\bar{b} / \emptyset, \mathfrak{M})$ if and only if there is $f \in \operatorname{Aut}(\mathfrak{M})$ mapping $\bar{a}$ to $\bar{b}$. Also for an arbitrary set $A$ we write $\operatorname{tp}^{\mathrm{g}}(\bar{a} / A, \mathfrak{M})=\operatorname{tp}^{\mathrm{g}}(\bar{b} / A, \mathfrak{M})$ if and only if there is $f \in \operatorname{Aut}(\mathfrak{M} / A)$ mapping $\bar{a}$ to $\bar{b}$, where

$$
\operatorname{Aut}(\mathfrak{M} / A)=\{f \in \operatorname{Aut}(\mathfrak{M}): f\lceil A \text { is the identity }\}
$$

We define another notion of type, called the weak type, by $\operatorname{tp}^{\mathrm{w}}(\bar{a} / A, \mathfrak{M})=$ $\operatorname{tp}^{\mathrm{w}}(\bar{b} / A, \mathfrak{M})$ if for each finite $A_{0} \subset A, \operatorname{tp}^{\mathrm{g}}\left(\bar{a} / A_{0}, \mathfrak{M}\right)=\operatorname{tp}^{\mathrm{g}}\left(\bar{b} / A_{0}, \mathfrak{M}\right)$.

REMARK 2.3. Let $A$ and sequences $I, J$ be bounded in a monster model $\mathfrak{M}$. Let also a monster model $\mathfrak{M}^{\prime}$ extend $\mathfrak{M}$. Then there is $f \in \operatorname{Aut}(\mathfrak{M} / A)$ sending $I$ to $J$ if and only if there is $g \in \operatorname{Aut}\left(\mathfrak{M}^{\prime} / A\right)$ sending $I$ to $J$.

By Remark 2.3, if $\bar{a}, \bar{b} \in \mathfrak{M}, A \subset \mathfrak{M}$ is $\mathfrak{M}$-bounded and $\mathfrak{M} \preccurlyeq \mathbb{K} \mathfrak{M}^{\prime}$ are monster models, then

$$
\operatorname{tp}^{\mathrm{g}}(\bar{a} / A, \mathfrak{M})=\operatorname{tp}^{\mathrm{g}}(\bar{b} / A, \mathfrak{M})
$$


if and only if

$$
\operatorname{tp}^{\mathrm{g}}\left(\bar{a} / A, \mathfrak{M}^{\prime}\right)=\operatorname{tp}^{\mathrm{g}}\left(\bar{b} / A, \mathfrak{M}^{\prime}\right)
$$

Since we assume that all the sets under discussion are bounded subsets of the monster model, we write only $\operatorname{tp}^{\mathrm{g}}(\bar{a} / A)$ for Galois type and $\operatorname{tp}^{\mathrm{w}}(\bar{a} / A)$ for weak type.

For any $\mathfrak{M}$-bounded ordinal $\alpha$, we say that a sequence $\left(\bar{a}_{i}\right)_{i<\alpha}$ of tuples is strongly $A$-indiscernible in $\mathfrak{M}$ if for any $\mathfrak{M}$-bounded ordinal $\beta \geq \alpha$ we can extend the sequence to $\left(\bar{a}_{i}\right)_{i<\beta}$ such that for any partial order-preserving $f$ : $\beta \rightarrow \beta$ we can find $F \in \operatorname{Aut}(\mathfrak{M} / A)$ mapping $\bar{a}_{i}$ to $\bar{a}_{f(i)}$ for each $i \in \operatorname{dom}(f)$.

The proof of the following lemma is skipped, but it is proved as Proposition 2.13 in [12].

Lemma 2.4 (Shelah). For every $\mathfrak{M}$-bounded cardinal $\kappa$ there exists a cardinal $\mathrm{H}(\kappa)$ such that the following holds. Whenever $A$ is a set of size $\kappa$ and $\left(\bar{a}_{i}\right)_{i<\mathrm{H}(\kappa)} \subset \mathfrak{M}$ are distinct tuples, there exists a strongly $A$-indiscernible sequence $\left(\bar{b}_{i}\right)_{i<\omega}$ in $\mathfrak{M}$ such that for each $n<\omega$ there are $i_{0}<\cdots<i_{n}<$ $\mathrm{H}(\kappa)$ such that

$$
\operatorname{tp}^{\mathrm{g}}\left(\bar{b}_{0}, \ldots, \bar{b}_{n} / A\right)=\operatorname{tp}^{\mathrm{g}}\left(\bar{a}_{i_{0}}, \ldots, \bar{a}_{i_{n}} / A\right) .
$$

Furthermore, if $I$ is any linear ordering, there exists a monster model $\mathfrak{M}^{\prime}$ extending $\mathfrak{M}$ and $\left(\bar{a}_{i}\right)_{i \in I}$ in $\mathfrak{M}^{\prime}$ such that for any $n<\omega$ and $j_{0}<\cdots<j_{n} \in I$ there are $i_{0}<\cdots<i_{n}<\mathrm{H}(\kappa)$ such that

$$
\operatorname{tp}^{\mathrm{g}}\left(\bar{b}_{0}, \ldots, \bar{b}_{n} / A\right)=\operatorname{tp}^{\mathrm{g}}\left(\bar{a}_{i_{0}}, \ldots, \bar{a}_{i_{n}} / A\right) .
$$

We write $\mathrm{H}\left(\aleph_{0}\right)=\mathrm{H}$. We know that $\mathrm{H}=\beth_{\left(2^{\aleph_{0}}\right)^{+}}$, which is the so called Hanf number of abstract elementary classes with $\mathrm{LS}(\mathbb{K})=\aleph_{0}$. We will always assume that the cardinal $\mu$ related to the monster model is closed under the operation $\mathrm{H}(\cdot)$, that is, when a set $A$ is bounded in $\mathfrak{M}$, also the cardinal $\mathrm{H}(|A|)$ is bounded in $\mathfrak{M}$. We can find arbitrarily large such cardinals: for any $\kappa$, define $\mu_{0}=\kappa$ and $\mu_{n+1}=\mathrm{H}\left(\mu_{n}\right)$. If $\mu=\bigcup_{n<\omega} \mu_{n}$, then $\lambda<\mu$ implies $\mathrm{H}(\lambda)<\mu$.

Now we see that also the notion of any finitely many tuples being included in a strongly $A$-indiscernible sequence is independent of the monster model for bounded $A$. Let $\left(\bar{a}_{0}, \ldots, \bar{a}_{n}\right)$ be included in some strongly $A$-indiscernible sequence $\left(\bar{a}_{i}\right)_{i<\alpha}$ in $\mathfrak{M}$. We can extend this sequence to the bounded length $\mathrm{H}(|A|)$. Then in any monster model $\mathfrak{M}^{\prime}$ such that $\mathfrak{M} \preccurlyeq \mathbb{K} \mathfrak{M}^{\prime}$, there is a strongly indiscernible $\left(\bar{b}_{i}\right)_{i<\lambda}$ and $i_{0}<\cdots<i_{n}<\mathrm{H}(|A|)$ such that

$$
\operatorname{tp}^{\mathrm{g}}\left(\bar{b}_{0}, \ldots, \bar{b}_{n} / A\right)=\operatorname{tp}^{\mathrm{g}}\left(\bar{a}_{i_{0}}, \ldots, \bar{a}_{i_{n}} / A\right)=\operatorname{tp}^{\mathrm{g}}\left(\bar{a}_{0}, \ldots, \bar{a}_{n} / A\right) .
$$

Thus we have $f \in \operatorname{Aut}\left(\mathfrak{M}^{\prime} / A\right)$ mapping $\bar{b}_{k}$ to $\bar{a}_{k}$ for each $0 \leq k \leq n$. The sequence $\left(f\left(\bar{b}_{i}\right)\right)_{i<\lambda}$ is strongly indiscernible in the extended monster model.

Similarly, Lemma 2.4 implies that if there are more than $\mathrm{H}(|A|)$-many distinct tuples, then for any $n<\omega$ we can find some $n$ of these tuples such that they are the beginning of a strongly $A$-indiscernible sequence. 
We say that a weak type $\operatorname{tp}^{\mathrm{w}}(\bar{a} / A)$ Lascar-splits over a finite $E \subset A$ if there is a strongly $E$-indiscernible sequence $\left(\bar{a}_{i}\right)_{i<\omega}$ such that $\bar{a}_{0}, \bar{a}_{1}$ are in $A$ and $\operatorname{tp}^{\mathrm{w}}\left(\bar{a}_{0} / \bar{a} \cup E\right) \neq \operatorname{tp}^{\mathrm{w}}\left(\bar{a}_{1} / \bar{a} \cup E\right)$. The notion of Lascar-splitting is also independent of the monster model. We define our notion of independence with built-in extension property.

Definition 2.5. We say that $\bar{a}$ is independent of $B$ over $A$, written

$$
\bar{a} \downarrow_{A} B,
$$

if there is a finite $E \subset A$ such that for all monster models $\mathfrak{M}^{\prime}$ extending $\mathfrak{M}$ and $D \subset \mathfrak{M}^{\prime}$ such that $A \cup B \subset D$ there is a monster model $\mathfrak{M}^{\prime \prime}$ extending $\mathfrak{M}^{\prime}$ and $\bar{b} \in \mathfrak{M}^{\prime \prime}$ such that $\operatorname{tp}^{\mathrm{w}}(\bar{b} / A \cup B)=\operatorname{tp}^{\mathrm{w}}(\bar{a} / A \cup B)$ and $\operatorname{tp}^{\mathrm{w}}(\bar{b} / D)$ does not Lascar-split over $E$.

If $\mathfrak{M} \preccurlyeq \mathbb{K} \mathfrak{M}^{\prime}$ are monster models and $\bar{a}, A \cup B \subset \mathfrak{M}$ are bounded, then $\bar{a} \downarrow_{A} B$ in $\mathfrak{M}$ if and only if $\bar{a} \downarrow_{A} B$ in $\mathfrak{M}^{\prime}$. Also by $\mu$-saturation, if $D$ in the above definition is $\mathfrak{M}$-bounded, then we can find $\bar{b}$ in $\mathfrak{M}$.

We study several monster models instead of one, since we want to be exact with our notion of boundedness. Usually the monster model is only assumed to be "large enough" and all sets in question "small enough", but we want to be clear with the details. The main difficulty with only one monster model would be in the proof of Proposition 2.7, where we would have to assume that a bounded union of bounded sets is also bounded. On the other hand, we want the least unbounded cardinal in $\mathfrak{M}$ to be a limit, and hence it would have to be a regular limit cardinal. It is consistent with ZFC that such cardinals do not exist above $\aleph_{0}$.

The following properties are clear by the definition.

Proposition 2.6.

(1) Invariance: Assume that $f$ is an automorphism of $\mathfrak{M}, \bar{a}, A, B \subset \mathfrak{M}$ are bounded and $\bar{a} \downarrow_{A} B$. Then $f(\bar{a}) \downarrow_{f(A)} f(B)$. Also if $\operatorname{tp}^{\mathrm{w}}(\bar{b} / B)=$ $\operatorname{tp}^{\mathrm{w}}(\bar{a} / B)$, then $\bar{b} \downarrow_{A} B$.

(2) Monotonicity: Assume that $A \subset B \subset C \subset D$ and $\bar{a} \downarrow_{A} D$. Then $\bar{a} \downarrow_{B} C$.

(3) Restricted local character: Assume that $\bar{a} \downarrow_{A} B$. Then there is a finite $E \subset A$ such that $\bar{a} \downarrow_{E}(A \cup B)$.

Now we see that "built-in extension" truly gives us the extension property.

Proposition 2.7 (Extension). Assume that $\bar{a} \downarrow_{A} B$ and $A \subset B \subset D$ (where all the sets are bounded in $\mathfrak{M})$. Then there exists $\bar{a}^{\prime}(\in \mathfrak{M})$ such that $\operatorname{tp}^{\mathrm{w}}(\bar{a} / B)=\operatorname{tp}^{\mathrm{w}}\left(\bar{a}^{\prime} / B\right)$ and $\bar{a}^{\prime} \downarrow_{A} D$.

Proof. By Proposition 2.6(3), we may assume that $A$ is finite. Enumerate all types $\operatorname{tp}^{\mathrm{w}}\left(\bar{b}_{i} / D\right), i<\kappa($ in $\mathfrak{M})$, such that $\operatorname{tp}^{\mathrm{w}}\left(\bar{b}_{i} / B\right)=\operatorname{tp}^{\mathrm{w}}(\bar{a} / B)$ and 
$\operatorname{tp}^{\mathrm{w}}\left(\bar{b}_{i} / D\right)$ does not Lascar-split over $A$ for all $i<\kappa$. This set is nonempty, since $\bar{a} \downarrow_{A} B$. For each $i<\kappa$, if $\bar{b}_{i} \downarrow_{A} D$, let $E_{i} \subset \mathfrak{M}_{i}$ be a set witnessing this, i.e. some set in some monster-extension $\mathfrak{M}_{i}$ such that $D \subset E_{i}$ and $\operatorname{tp}^{\mathrm{w}}\left(\bar{b}_{i} / D\right)$ does not have a non-splitting extension to $E_{i}$. If $\bar{b}_{i} \downarrow_{A} D$, let $E_{i}=D$.

The set $E=\bigcup_{i<\kappa} E_{i}$ is bounded in some monster-extension $\mathfrak{M}^{\prime}$. But $\bar{a} \downarrow_{A} B$ also in $\mathfrak{M}^{\prime}$, and thus there is $\bar{b} \in \mathfrak{M}^{\prime}$ such that $\operatorname{tp}^{\mathrm{w}}(\bar{b} / B)=\operatorname{tp}^{\mathrm{w}}(\bar{a} / B)$ and $\operatorname{tp}^{\mathrm{w}}(\bar{b} / E)$ does not Lascar-split over $A$. Now $\operatorname{tp}^{\mathrm{w}}(\bar{b} / D)$ does not Lascarsplit over $A$ either, and by $\mu$-saturation, there is $i<\kappa$ such that $\operatorname{tp}^{\mathrm{w}}(\bar{b} / D)=$ $\operatorname{tp}^{\mathrm{w}}\left(\bar{b}_{i} / D\right)$. But tp $\mathrm{w}^{\mathrm{w}}\left(\bar{b}_{i} / D\right)$ has a non-splitting extension to $E_{i}$, namely $\bar{b}$, and thus $\bar{b}_{i} \downarrow_{A} D$, and $\bar{b}_{i}$ is as required.

We use both notations $\bar{a} \bar{b}$ and $\bar{a}^{\frown} \bar{b}$ for the concatenation of tuples. We can also abbreviate $\bar{a} \cup \bar{b}$ for $\{\bar{a}\} \cup\{\bar{b}\}$ and $\bar{a} \in A$ for $\bar{a} \in A^{\lg (\bar{a})}$.

Proposition 2.8 (Finite Pairs Lemma). Let $B$ be finite and $A \subset B$. Assume that $\bar{a} \downarrow_{A} B$ and $\bar{b} \downarrow_{A \cup \bar{a}} B \cup \bar{a}$. Then $\bar{a}^{\frown} \bar{b} \downarrow_{A} B$.

Proof. Assume, for contradiction, that $\bar{a}\urcorner \bar{b} \downarrow_{A} B$. In particular, the finite set $A$ does not witness that $\bar{a}^{\frown} \bar{b} \downarrow_{A} B$. Hence, there is $D$ containing $B$ such that whenever $\operatorname{tp}^{\mathrm{w}}\left(\bar{a}^{\prime}, \bar{b}^{\prime} / B\right)=\operatorname{tp}^{\mathrm{w}}(\bar{a}, \bar{b} / B)$, then $\operatorname{tp}^{\mathrm{w}}\left(\bar{a}^{\prime}, \bar{b}^{\prime} / D\right)$ Lascar-splits over $A$. We may increase the set $D$ if necessary, and assume that it has the following property: For every finite $A \subset D$ and tuples $\bar{a}_{0}, \bar{a}_{1} \in D$ such that they are a beginning of a strongly $A$-indiscernible sequence $\left(\bar{a}_{i}\right)_{i<\mathrm{H}}$, there is one such sequence in $D$.

By definition there is $\bar{a}^{\prime} \in \mathfrak{M}^{\prime}$ such that $\operatorname{tp}^{\mathrm{w}}\left(\bar{a}^{\prime} / B\right)=\operatorname{tp}^{\mathrm{w}}(\bar{a} / B)$ and $\operatorname{tp}^{\mathrm{w}}\left(\bar{a}^{\prime} / D\right)$ does not Lascar-split over $A$. Since $B$ is finite, we have $f \in$ Aut $\left(\mathfrak{M}^{\prime} / B\right)$ such that $f(\bar{a})=\bar{a}^{\prime}$. Now $\operatorname{tp}^{\mathrm{w}}\left(\bar{a}^{\prime}, f(\bar{b}) / B\right)=\operatorname{tp}^{\mathrm{w}}(\bar{a}, \bar{b} / B)$, and thus $f(\bar{b}) \downarrow_{A \cup \bar{a}^{\prime}} B \cup \bar{a}^{\prime}$. Again by definition there is $\bar{b}^{\prime} \in \mathfrak{M}^{\prime}$ such that $\operatorname{tp}^{\mathrm{w}}\left(\bar{b}^{\prime} / B \cup \bar{a}^{\prime}\right)=\operatorname{tp}^{\mathrm{w}}\left(f(\bar{b}) / B \cup \bar{a}^{\prime}\right)$ and $\operatorname{tp}^{\mathrm{w}}\left(\bar{b}^{\prime} / D \cup \bar{a}^{\prime}\right)$ does not Lascar-split over $A \cup \bar{a}^{\prime}$. Hence also $\operatorname{tp}^{\mathrm{w}}\left(\bar{a}^{\prime}, \bar{b}^{\prime} / B\right)=\operatorname{tp}^{\mathrm{w}}\left(\bar{a}^{\prime}, f(\bar{b}) / B\right)=\operatorname{tp}^{\mathrm{w}}(\bar{a}, \bar{b} / B)$.

Let $\left(\bar{c}_{i}\right)_{i<\omega}$ be strongly $A$-indiscernible such that $\operatorname{tp}^{\mathrm{g}}\left(\bar{c}_{0} / A \cup \bar{a}^{\prime} \cup \bar{b}^{\prime}\right) \neq$ $\operatorname{tp}^{\mathrm{g}}\left(\bar{c}_{1} / A \cup \bar{a}^{\prime} \cup \bar{b}^{\prime}\right)$ and $\bar{c}_{0}, \bar{c}_{1} \in D$. By strong indiscernibility, this sequence extends to a strongly $A$-indiscernible $\left(\bar{c}_{i}\right)_{i<\mathrm{H}}$. By the above property of $D$, we may assume that $\left(\bar{c}_{i}\right)_{i<\mathrm{H}}$ is in $D$. Since there are either H-many $\bar{c}_{i}$ not realizing $\operatorname{tp}^{\mathrm{g}}\left(\bar{c}_{0} / A \cup \bar{a}^{\prime} \cup \bar{b}^{\prime}\right)$ or $\mathrm{H}$-many $\bar{c}_{i}$ not realizing $\operatorname{tp}^{\mathrm{g}}\left(\bar{c}_{1} / A \cup \bar{a}^{\prime} \cup \bar{b}^{\prime}\right)$, we may assume that

$$
\operatorname{tp}^{\mathrm{g}}\left(\bar{c}_{0} / A \cup \bar{a}^{\prime} \cup \bar{b}^{\prime}\right) \neq \operatorname{tp}^{\mathrm{g}}\left(\bar{c}_{i} / A \cup \bar{a}^{\prime} \cup \bar{b}^{\prime}\right)
$$

for each $i<\mathrm{H}$.

We claim that $\left(\bar{c}_{i}\right)_{i<\mathrm{H}}$ has the property that for any $i_{0}<i_{1}<\mathrm{H}$,

$$
\operatorname{tp}^{\mathrm{w}}\left(\bar{c}_{i_{0}}, \bar{c}_{i_{1}} / A \cup \bar{a}^{\prime}\right)=\operatorname{tp}^{\mathrm{w}}\left(\bar{c}_{0}, \bar{c}_{1} / A \cup \bar{a}^{\prime}\right) .
$$

Assume, for contradiction, that there are $i_{0}<i_{1}$ such that the above does not hold. We check the following three possibilities: 
(1) $1<i_{0}$

(2) $i_{0}=0$,

(3) $i_{0}=1$.

Assume that (1) holds. Since $\mathrm{H}$ is an infinite cardinal, we may skip fewer than $\mathrm{H}$-many tuples if necessary and assume that $i_{0}=2$ and $i_{1}=3$. The sequence $\left(\bar{d}_{i}\right)_{i<\mathrm{H}}$, where $\bar{d}_{i}=\left(\bar{c}_{\alpha+2 n}, \bar{c}_{\alpha+2 n+1}\right)$ for $i=\alpha+n<\mathrm{H}, \alpha$ limit and $n<\omega$, is strongly $A$-indiscernible and $\operatorname{tp}^{\mathrm{g}}\left(\bar{d}_{0} / A \cup \bar{a}^{\prime}\right) \neq \operatorname{tp}^{\mathrm{g}}\left(\bar{d}_{1} / A \cup \bar{a}^{\prime}\right)$. Then we find that $\operatorname{tp}^{\mathrm{w}}\left(\bar{a}^{\prime} / D\right)$ Lascar-splits over $A$, a contradiction. If we have $(2)$, then the sequence $\left(\bar{c}_{0}, \bar{c}_{i}\right)_{i<\mathrm{H}}$ is strongly $A$-indiscernible with tp ${ }^{\mathrm{w}}\left(\bar{c}_{0}, \bar{c}_{i_{1}} / A \cup \bar{a}^{\prime}\right) \neq$ $\operatorname{tp}^{\mathrm{w}}\left(\bar{c}_{0}, \bar{c}_{1} / A \cup \bar{a}^{\prime}\right)$. We infer again that $\operatorname{tp}^{\mathrm{w}}\left(\bar{a}^{\prime} / D\right)$ Lascar-splits over $A$, a contradiction.

Assume that (1) or (2) does not hold, and thus for all indices $i_{0}<i_{1}$ such that $\operatorname{tp}^{\mathrm{w}}\left(\bar{c}_{i_{0}}, \bar{c}_{i_{1}} / A \cup \bar{a}^{\prime}\right) \neq \mathrm{tp}^{\mathrm{w}}\left(\bar{c}_{0}, \bar{c}_{1} / A \cup \bar{a}^{\prime}\right)$, we have $i_{0}=1$. We can study the strongly $A$-indiscernible sequence $\left(\bar{c}_{i}\right)_{i<\mathrm{H}, i \neq 1}$, since $\operatorname{tp}^{\mathrm{g}}\left(\bar{c}_{0} / A \cup \bar{a}^{\prime} \cup \bar{b}^{\prime}\right) \neq$ $\operatorname{tp}^{\mathrm{g}}\left(\bar{c}_{2} / A \cup \bar{a}^{\prime} \cup \bar{b}^{\prime}\right)$ and $\bar{c}_{0}, \bar{c}_{2} \in D$. The claim holds for this sequence.

We have shown the claim. Now by Lemma 2.4, there is a strongly $\left(A \cup \bar{a}^{\prime}\right)$ indiscernible sequence $\left(\bar{c}_{i}^{\prime}\right)_{i<\omega}$ such that $\operatorname{tp}\left(\bar{c}_{0}^{\prime}, \bar{c}_{1}^{\prime} / A \cup \bar{a}^{\prime}\right)=\operatorname{tp}\left(\bar{c}_{i_{0}}, \bar{c}_{i_{1}} / A \cup \bar{a}\right)$ for some $i_{0}<i_{1}<\mathrm{H}$. By the previous claim we have $f \in \operatorname{Aut}\left(\mathfrak{M}^{\prime} / A \cup \bar{a}^{\prime}\right)$ mapping $\left(\bar{c}_{0}^{\prime}, \bar{c}_{1}^{\prime}\right)$ to $\left(\bar{c}_{0}, \bar{c}_{1}\right)$ and thus may assume that $\bar{c}_{0}^{\prime}=\bar{c}_{0}$ and $\bar{c}_{1}^{\prime}=\bar{c}_{1}$. Since $\operatorname{tp}^{\mathrm{g}}\left(\bar{c}_{0} /\left(A \cup \bar{a}^{\prime}\right) \cup \bar{b}^{\prime}\right) \neq \operatorname{tp}^{\mathrm{g}}\left(\bar{c}_{1} /\left(A \cup \bar{a}^{\prime}\right) \cup \bar{b}^{\prime}\right)$, it follows that $\operatorname{tp}^{\mathrm{w}}\left(\bar{b}^{\prime} / D \cup \bar{a}^{\prime}\right)$ Lascar-splits over $A \cup \bar{a}^{\prime}$, a contradiction.

Let $A \subset \mathfrak{M}$ be a bounded subset and $\bar{a} \in \mathfrak{M}$ a tuple. We say that $\operatorname{tp}^{\mathrm{w}}(\bar{a} / A)$ is bounded if the set $\left\{\bar{b} \in \mathfrak{M}: \bar{b} \models \operatorname{tp}^{\mathrm{w}}(\bar{a} / A)\right\}$ is bounded in $\mathfrak{M}$. We see that if $\operatorname{tp}^{\mathrm{w}}(\bar{a} / A)$ is bounded, then

$$
\left\{\bar{b} \in \mathfrak{M}: \bar{b}=\operatorname{tp}^{\mathrm{w}}(\bar{a} / A)\right\}<\mathrm{H}(|A|) .
$$

Namely, if the set had some bounded size $\kappa \geq \mathrm{H}(|A|)$, we could find a strongly $A$-indiscernible sequence of distinct tuples realizing $\operatorname{tp}^{\mathrm{w}}(\bar{a} / A)$. Hence by strong indiscernibility, there would be at least $\kappa^{+}$-many tuples in $\mathfrak{M}$ realizing $\operatorname{tp}^{\mathrm{w}}(\bar{a} / A)$. This is a contradiction. Since in any monster model $\mathrm{H}(|A|)$ is bounded if and only if $A$ is bounded, a type $\operatorname{tp}^{\mathrm{w}}(\bar{a} / A)$ is bounded in $\mathfrak{M}$ if and only if it is bounded in all extending monster models. Also by $\mu$-saturation, if $A$ is bounded in $\mathfrak{M}, \operatorname{tp}^{\mathrm{w}}(\bar{a} / A)$ is bounded and $\mathfrak{M}^{\prime}$ is a monster model extending $\mathfrak{M}$, then $\left\{\bar{b} \in \mathfrak{M}: \bar{b} \models \operatorname{tp}^{\mathrm{w}}(\bar{a} / A)\right\}=\left\{\bar{b} \in \mathfrak{M}^{\prime}: \bar{b}=\right.$ $\left.\operatorname{tp}^{\mathrm{w}}(\bar{a} / A)\right\}$.

\section{LEMMA 2.9. Let $A$ be finite.}

(1) If $\operatorname{tp}^{\mathrm{w}}(\bar{a} / A)$ is bounded, then $\bar{a} \downarrow_{A} B$ for any $B$.

(2) If $\operatorname{tp}^{\mathrm{w}}(\bar{a} / A)$ is not bounded, then $\bar{a} \downarrow_{A} \bar{a}$.

Proof. Let $\mathfrak{M}^{\prime}$ be any monster-extension and $D \subset \mathfrak{M}^{\prime}$ any set. Assume that $\operatorname{tp}^{\mathrm{w}}(\bar{a} / B)$ does split over $A$. Let $\left(\bar{b}_{i}\right)_{i<\mathrm{H}}$ be strongly $A$-indiscernible 
such that $\operatorname{tp}^{\mathrm{g}}\left(\bar{b}_{0} / A \cup\{\bar{a}\}\right) \neq \operatorname{tp}^{\mathrm{g}}\left(\bar{b}_{1} / A \cup\{\bar{a}\}\right)$. There has to be either Hmany $i$ such that $\operatorname{tp}^{\mathrm{g}}\left(\bar{b}_{i} / A \cup\{\bar{a}\}\right) \neq \operatorname{tp}^{\mathrm{g}}\left(\bar{b}_{0} / A \cup\{\bar{a}\}\right)$ or H-many $i$ such that $\operatorname{tp}^{\mathrm{g}}\left(\bar{b}_{i} / A \cup\{\bar{a}\}\right) \neq \operatorname{tp}^{\mathrm{g}}\left(\bar{b}_{1} / A \cup\{\bar{a}\}\right)$. Thus we may assume $\operatorname{tp}^{\mathrm{w}}\left(\bar{b}_{i} / A \cup\{\bar{a}\}\right)$ $\neq \operatorname{tp}^{\mathrm{w}}\left(\bar{b}_{0} / A \cup\{\bar{a}\}\right)$ for all $0<i<\mathrm{H}$. By strong $A$-indiscernibility, for each $i<\mathrm{H}$, there is $f_{i} \in \operatorname{Aut}\left(\mathfrak{M}^{\prime} / A\right)$ such that $f_{i}\left(\bar{b}_{k}\right)=\bar{b}_{i+k}$ for all $k<\mathrm{H}$. Now if $i<j$ then $f_{i}(\bar{a}) \neq f_{j}(\bar{a})$. Otherwise we would have $\left(f_{i}{ }^{-1} \circ f_{j}\right)(\bar{a})=\bar{a}$ and $\left(f_{i}^{-1} \circ f_{j}\right)\left(\bar{b}_{0}\right)=\bar{b}_{k}$ for $k>0$. Now $\left(f_{i}(\bar{a})\right)_{i<\mathrm{H}}$ are different realizations of $\operatorname{tp}^{\mathrm{w}}(\bar{a} / A)$, and the type $\operatorname{tp}^{\mathrm{w}}(\bar{a} / A)$ is not bounded. Thus if $\operatorname{tp}^{\mathrm{w}}(\bar{a} / A)$ is bounded, $\operatorname{tp}^{\mathrm{w}}(\bar{a} / D)$ does not split over $A$ for any $D$ or monster-extension $\mathfrak{M}^{\prime}$. This proves (1).

To prove (2), assume that $\operatorname{tp}^{\mathrm{w}}(\bar{a}, A)$ is not bounded. There are H-many tuples $\bar{b}$ such that $\operatorname{tp}^{\mathrm{g}}(\bar{b} / A)=\operatorname{tp}^{\mathrm{g}}(\bar{a} / A)$. By Lemma 2.4, there is a strongly $A$-indiscernible sequence $\left(\bar{a}_{i}\right)_{i<\omega}$ of distinct tuples such that $\operatorname{tp}^{\mathrm{g}}\left(\bar{a}_{0} / A\right)=$ $\operatorname{tp}^{\mathrm{g}}(\bar{a} / A)$ and hence $\operatorname{tp}^{\mathrm{g}}\left(\bar{a}_{i} / A\right)=\operatorname{tp}^{\mathrm{g}}(\bar{a} / A)$ for each $i<\omega$. Furthermore, since we have $f \in \operatorname{Aut}(\mathfrak{M} / A)$ mapping $\bar{a}_{0}$ to $\bar{a}$, we may assume that $\bar{a}_{0}=\bar{a}$. Assume, for contradiction, that $\bar{a} \downarrow_{A} \bar{a}$. Let $\bar{a}^{\prime}$ be such that $\operatorname{tp}^{\mathrm{w}}\left(\bar{a}^{\prime} / A \cup\{\bar{a}\}\right)$ $=\operatorname{tp}^{\mathrm{w}}(\bar{a} / E \cup\{\bar{a}\})$ and the type $\operatorname{tp}^{\mathrm{w}}\left(\bar{a}^{\prime} / A \cup\left\{\bar{a}_{i}: i<\omega\right\}\right)$ does not Lascarsplit over $A$. But now we must have $\bar{a}^{\prime}=\bar{a}$ and this is a contradiction, since $\operatorname{tp}^{\mathrm{g}}\left(\bar{a}_{0} / A \cup\{\bar{a}\}\right) \neq \operatorname{tp}^{q}\left(\bar{a}_{1} / A \cup\{\bar{a}\}\right)$ and thus $\operatorname{tp}^{\mathrm{w}}\left(\bar{a} / A \cup\left\{\bar{a}_{i}: i<\omega\right\}\right)$ does Lascar-split over $A$. This proves (2).

Proposition 2.10. Let $A \subset B$ be finite, $\bar{a} \downarrow_{A} B$ and $B \subset D$. There is $\bar{a}^{\prime}$ such that $\left(\bar{a}, \bar{a}^{\prime}\right)$ is a beginning of a strongly $B$-indiscernible sequence and $\bar{a}^{\prime} \downarrow_{A} D$.

Proof. If $\operatorname{tp}^{\mathrm{w}}(\bar{a} / A)$ is bounded, we can take the constant sequence, which is strongly $A$-indiscernible, by Lemma $2.9(1)$. We assume that $\operatorname{tp}^{\mathrm{w}}(\bar{a} / A)$ is unbounded. By extension there are $\bar{a}_{i}$ for $i<\mathrm{H}$ such that $\operatorname{tp}^{\mathrm{w}}\left(\bar{a}_{i} / B\right)=$ $\operatorname{tp}^{\mathrm{w}}(\bar{a} / B)$ and $\bar{a}_{i} \downarrow_{A} B \cup \bigcup_{j<i}\left\{\bar{a}_{j}\right\}$. By Lemma $2.9(2)$ and monotonicity we have $\bar{a}_{j} \neq \bar{a}_{i}$ for any $j \neq i$. Thus there are $j_{0}, j_{1}<\mathrm{H}$ such that $\left(\bar{a}_{j_{0}}, \bar{a}_{j_{1}}\right)$ is a beginning of a strongly $B$-indiscernible sequence. Since $B$ is finite, there is $f \in \operatorname{Aut}(\mathfrak{M} / B)$ mapping $\bar{a}_{j_{0}}$ to $\bar{a}$. Define $\bar{a}^{*}=f\left(\bar{a}_{j_{1}}\right)$. Now $\left(\bar{a}, \bar{a}^{*}\right)$ is the beginning of a strongly $B$-indiscernible sequence and $\bar{a}^{*} \downarrow_{A} B \cup\{\bar{a}\}$. Again by extension there is $\bar{a}^{\prime}$ such that $\bar{a}^{\prime} \downarrow_{A} D$ and $\operatorname{tp}^{\mathrm{w}}\left(\bar{a}^{\prime} / B \cup\{\bar{a}\}\right)=$ $\operatorname{tp}^{\mathrm{w}}\left(\bar{a}^{*} / B \cup\{\bar{a}\}\right)$. Let $g \in \operatorname{Aut}(B \cup\{\bar{a}\})$ be such that $g\left(\bar{a}^{*}\right)=\bar{a}^{\prime}$. Then also $\left(g(\bar{a}), g\left(\bar{a}^{*}\right)\right)=\left(\bar{a}, \bar{a}^{\prime}\right)$ is a beginning of a strongly $B$-indiscernible sequence.

2.1. Lascar strong types. We say that an equivalence relation $E$ in a monster model $\mathfrak{M}$ is $A$-invariant if it is preserved by each $f \in \operatorname{Aut}(\mathfrak{M} / A)$. We also say that an equivalence relation $E$ is bounded if the number of equivalence classes of $E$ in $\mathfrak{M}$ is bounded. If $E$ is a bounded and $A$-invariant equivalence relation and $\left(\bar{a}_{i}\right)_{i<\omega}$ a strongly $A$-indiscernible sequence, then $E\left(\bar{a}_{i_{0}}, \bar{a}_{i_{1}}\right)$ for each $i_{0}, i_{1}<\omega$. Otherwise due to $A$-invariance we would 
get $\neg E\left(\bar{a}_{i}, \bar{a}_{j}\right)$ for each $\bar{a}_{i}, \bar{a}_{j}$ in the sequence. If the number of equivalence classes of $E$ is $\kappa$, we could extend the sequence to the length of $\kappa^{+}$, and get a contradiction.

We conclude that if an $A$-invariant equivalence relation has a bounded number of equivalence classes in $\mathfrak{M}$, the number must be strictly less than $\mathrm{H}(|A|)$. Also by $\mu$-saturation it cannot have any other equivalence classes in any extending monster model.

Definition 2.11. We say that sequences $\bar{a}$ and $\bar{b}$ have the same Lascar strong type over $A$, written

$$
\operatorname{Lstp}(\bar{a} / A)=\operatorname{Lstp}(\bar{b} / A)
$$

if $\ell(\bar{a})=\ell(\bar{b})$ and $E(\bar{a}, \bar{b})$ holds for any $A$-invariant and bounded equivalence relation $\mathbf{E}$ of $\ell(\bar{a})$-tuples.

Each tuple in a strongly $A$-indiscernible sequence has the same Lascar strong type over $A$. Thus the number of Lascar strong types over a set $A$ is strictly smaller than the cardinal $\mathrm{H}(|A|)$. This holds by Lemma 2.4, since for any sequence $\left(\bar{a}_{i}\right)_{i<\kappa}$ for $\kappa \geq \mathrm{H}(A)$, there are indices $i<j<\kappa$ such that the tuples $\bar{a}_{i}, \bar{a}_{j}$ are in the same strongly $A$-indiscernible sequence. As a corollary of Proposition 2.10 we get the following.

Corollary 2.12. Let $A \subset B \subset D$ and $\bar{a} \downarrow_{A} B$, where $B$ is finite. Then there is $\bar{a}^{\prime}$ such that $\operatorname{Lstp}\left(\bar{a}^{\prime} / B\right)=\operatorname{Lstp}(\bar{a} / B)$ and $\bar{a}^{\prime} \downarrow_{A} D$.

We give an equivalent condition for two tuples to have the same Lascar strong type over $A$.

Proposition 2.13. The following are equivalent.

(1) $\operatorname{Lstp}(\bar{a} / A)=\operatorname{Lstp}(\bar{b} / A)$.

(2) There exists $n<\omega, \bar{a}_{i}$ for $i \leq n$ and strongly $A$-indiscernible sequences $J_{i}$ for $i<n$ such that $\bar{a}_{0}=\bar{a}, \bar{a}_{n}=\bar{b}$ and $\bar{a}_{i}, \bar{a}_{i+1} \in J_{i}$ for $i<n$.

Proof. Since elements in a strongly $A$-indiscernible sequence have the same Lascar strong types over $A,(2)$ implies (1). We show that (1) implies (2). It is enough to show that the relation defined by (2) is an $A$ invariant equivalence relation with a bounded number of classes. It is clearly $A$-invariant, transitive and symmetric. The trivial strongly $A$-indiscernible sequence $(\bar{a})_{i<\omega}$ shows that it is also reflexive. It remains to show that it is bounded. Assume that it would not be bounded, and thus there would be $\mathrm{H}(|A|)$-many inequivalent tuples. But by Lemma 2.4, at least two of these elements would be included in some strongly $A$-indiscernible sequence, a contradiction.

At least by the previous proposition it is clear that the relation $\operatorname{Lstp}(\bar{a} / A)$ does not depend on the possible extension of the monster model. 
If $\left(\bar{a}_{i}\right)_{i<\alpha}$ is a strongly $E \cup \bar{c}$-indiscernible sequence, the sequence $\left(\bar{a}_{i} \bar{c}\right)_{i<\alpha}$ is strongly $E$-indiscernible. The above proposition implies that

$$
\operatorname{Lstp}(\bar{a} / E \cup \bar{c})=\operatorname{Lstp}(\bar{b} / E \cup \bar{c}) \Rightarrow \operatorname{Lstp}(\bar{a} \bar{c} / E)=\operatorname{Lstp}(\bar{b} \bar{c} / E) .
$$

Definition 2.14. We say that $f \in \operatorname{Aut}(\mathfrak{M} / A)$ is a strong automorphism over $A$ if $\operatorname{Lstp}(\bar{a} / A)=\operatorname{Lstp}(f(\bar{a}) / A)$ for each tuple $\bar{a}$.

We define $\operatorname{Saut}(\mathfrak{M} / A)$ to be the group of strong automorphisms fixing $A$ pointwise. The group $\operatorname{Saut}(\mathfrak{M} / A)$ is a normal subgroup of the automorphism group $\operatorname{Aut}(\mathfrak{M} / A)$.

Proposition 2.15. The following are equivalent for a bounded $A$.

(1) $\operatorname{Lstp}(\bar{a} / A)=\operatorname{Lstp}(\bar{b} / A)$.

(2) There is $f \in \operatorname{Saut}\left(\mathfrak{M}^{\prime} / A\right)$ such that $f(\bar{a})=\bar{b}$.

Proof. By the definition of a strong automorphism, (2) implies (1). To prove that (1) implies (2), we show that the equivalence relation defined by (2) is $A$-invariant and has a bounded number of equivalence classes. First, it is $A$-invariant due to the normality of the subgroup $\operatorname{Saut}(\mathfrak{M} / A)$ of $\operatorname{Aut}(\mathfrak{M} / A)$. To prove that it is bounded, assume to the contrary that $\left(\bar{a}_{i}\right)_{i<\mathrm{H}(\mathrm{H}(|A|))}$ are distinct tuples. We remark that the cardinal $\mathrm{H}(\mathrm{H}(|A|))$ is bounded. Let $\mathscr{A}$ be a model of size $\mathrm{H}(|A|)$ such that $A \subset \mathscr{A}$ and each Lascar strong type over $A$ is represented in $\mathscr{A}$. By Lemma 2.4 there are $i_{0}<i_{1}<\mathrm{H}(\mathrm{H}(|A|))=\mathrm{H}(|\mathscr{A}|)$ such that $\left(\bar{a}_{i_{0}}, \bar{a}_{i_{1}}\right)$ is the beginning of a strongly $\mathscr{A}$-indiscernible sequence. Thus there is $f \in \operatorname{Aut}(\mathfrak{M} / \mathscr{A})$ mapping $\bar{a}_{i_{0}}$ to $\bar{a}_{i_{1}}$. We show that this automorphism is actually strong over $\mathscr{A}$, which implies that $\bar{a}_{i_{0}}$ and $\bar{a}_{i_{1}}$ are equivalent. For this, let $\bar{a} \in \mathfrak{M}$ be arbitrary. There is $\bar{a}^{\prime} \in \mathscr{A}$ realizing $\operatorname{Lstp}(\bar{a} / A)$. Since Lstp is an $A$-invariant notion and $f\left(\bar{a}^{\prime}\right)=\bar{a}^{\prime}$, we conclude that

$$
\operatorname{Lstp}(f(\bar{a}) / A)=\operatorname{Lstp}\left(f\left(\bar{a}^{\prime}\right) / A\right)=\operatorname{Lstp}\left(\bar{a}^{\prime} / A\right)=\operatorname{Lstp}(\bar{a} / A) .
$$

The above equivalence implies that if $\operatorname{Lstp}(\bar{a} / A)=\operatorname{Lstp}(\bar{b} / A)$ and $\bar{c}$ is an arbitrary tuple, we can always find $\bar{d} \operatorname{such}$ that $\operatorname{Lstp}(\bar{a} \bar{d} / A)=\operatorname{Lstp}(\bar{b} \bar{c} / A)$.

Lemma 2.16. Assume that $\mathscr{A}$ is an $\aleph_{0}$-saturated model. Then the following are equivalent.

(1) $\operatorname{tp}^{\mathrm{w}}(\bar{a} / \mathscr{A})$ Lascar-splits over finite $E \subset \mathscr{A}$.

(2) There are tuples $\bar{c}, \bar{d} \in \mathscr{A}$ such that $\operatorname{Lstp}(\bar{c} / E)=\operatorname{Lstp}(\bar{d} / E)$ but $\operatorname{tp}^{\mathrm{g}}(\bar{c} / E \cup \bar{a}) \neq \operatorname{tp}^{\mathrm{g}}(\bar{d} / E \cup \bar{a})$.

Proof. Item (1) implies (2) by the definition. We show that if (1) does not hold, then neither does $(2)$. For this, assume that $\operatorname{tp}^{\mathrm{w}}(\bar{a} / \mathscr{A})$ does not Lascar-split over $E$ and $\bar{c}, \bar{d}$ are distinct tuples in $\mathscr{A}$ such that

$$
\operatorname{Lstp}(\bar{c} / E)=\operatorname{Lstp}(\bar{d} / E) \text {. }
$$


By Proposition 2.13 there are strongly $E$-indiscernible sequences $I_{k}$ and tuples $\left(\bar{a}_{k}, \bar{a}_{k+1}\right) \in I_{k}$ for $0 \leq k \leq n$ such that $\bar{a}_{0}=\bar{c}$ and $\bar{a}_{n+1}=\bar{d}$. Since $\mathscr{A}$ is $\aleph_{0}$-saturated, we may assume that each $\bar{a}_{k}$ is in $\mathscr{A}$. But now, since $\operatorname{tp}^{\mathrm{w}}(\bar{a} / E)$ does not Lascar-split over $E$, we must have $\operatorname{tp}^{\mathrm{g}}\left(\bar{a}_{0} / E \cup \bar{a}\right)=$ $\operatorname{tp}^{\mathrm{g}}\left(\bar{a}_{1} / E \cup \bar{a}\right)=\cdots=\operatorname{tp}^{\mathrm{g}}\left(\bar{a}_{n} / E \cup \bar{a}\right)=\operatorname{tp}^{\mathrm{g}}\left(\bar{a}_{n+1} / E \cup \bar{a}\right)$.

We define that a model $\mathscr{A}$ is a-saturated if each Lascar strong type over a finite subset of $\mathscr{A}$ is realized in $\mathscr{A}$.

\subsection{Restricted properties with simplicity and weak stability.} We introduce new properties called weak stability and simplicity. We say that $(\mathbb{K}, \preccurlyeq \mathbb{K})$ is weakly stable in a cardinal $\lambda$ if whenever $|A| \leq \lambda$ and $\left(\bar{a}_{i}\right)_{i<\lambda^{+}}$ are tuples, there are $i<j<\lambda^{+}$such that $\operatorname{tp}^{\mathrm{w}}\left(\bar{a}_{i} / A\right)=\operatorname{tp}^{\mathrm{w}}\left(\bar{a}_{j} / A\right)$.

DEFINITION 2.17. We say that $(\mathbb{K}, \preccurlyeq \mathbb{K})$ is weakly stable if there is a cardinal $\lambda$ such that $(\mathbb{K}, \preccurlyeq \mathbb{K})$ is weakly stable in $\lambda$.

Definition 2.18. We say that $(\mathbb{K}, \preccurlyeq \mathbb{K})$ is simple if $\bar{a} \downarrow_{A} A$ for each tuple $\bar{a}$ and finite set $A$.

In the $\aleph_{0}$-stable case in [12] we defined simplicity as the assumption that for any $\bar{a}$ and arbitrary $A$ there is a finite $A^{\prime} \subset A$ such that $\bar{a} \downarrow_{A^{\prime}} A$. Here we call this property local character. With $\aleph_{0}$-stability, the above notion of simplicity is equivalent to local character $\left({ }^{2}\right)$.

In this section we collect those properties of the notion $\downarrow$ which we can derive from these restricted versions of simplicity and stability. From now on we will always assume that $(\mathbb{K}, \preccurlyeq \mathbb{K})$ is simple and weakly stable.

Weak stability and simplicity are needed to prove finite symmetry for $\downarrow$. Then we will use simplicity and finite symmetry to prove several other properties.

Proposition 2.19. Assume that $A$ is finite, $\bar{a} \downarrow_{A} \bar{b}$ and $\bar{b} \downarrow_{A} \bar{a}$. Then there are $\bar{a}_{i}, \bar{b}_{i}$ for $i<\mathrm{H}$ such that $\bar{b}_{i} \downarrow_{A} \bar{a}_{j}$ if and only if $i>j$.

Proof. Let $\bar{a}_{0}=\bar{a}$ and $\bar{b}_{0}=\bar{b}$. Define $\bar{a}_{i}, \bar{b}_{i}$ by induction such that

(1) $\operatorname{tp}^{\mathrm{w}}\left(\bar{a}_{i}^{\frown} \bar{b}_{i} / A\right)=\operatorname{tp}^{\mathrm{w}}(\bar{a} \frown \bar{b} / A)$ for all $i<\mathrm{H}$.

(2) $\operatorname{tp}^{\mathrm{w}}\left(\bar{a}_{i} / A \cup \bar{b}\right)=\operatorname{tp}^{\mathrm{w}}(\bar{a} / A \cup \bar{b})$ for all $i<\mathrm{H}$.

(3) The pair $\left(\bar{b}, \bar{b}_{i}\right)$ is a beginning of a strongly $A$-indiscernible sequence for each $0<i<\mathrm{H}$.

(4) $\bar{a}_{i}^{\frown} \bar{b}_{i} \downarrow_{A} B$ for every finite $B \subset \bigcup_{j<i}\left\{\bar{a}_{j}, \bar{b}_{j}\right\}$.

Note that due to simplicity, item (4) holds also when $i=0$. Assume we have defined $\bar{a}_{i}, \bar{b}_{i}$ for $i<\alpha$. Since $\bar{a} \downarrow_{A} \bar{b}$, by extension we get $\bar{a}_{\alpha}$ such that $\operatorname{tp}^{\mathrm{w}}\left(\bar{a}_{\alpha} / A \cup \bar{b}\right)=\operatorname{tp}^{\mathrm{w}}(\bar{a} / A \cup \bar{b})$ and $\bar{a}_{\alpha} \downarrow_{A} \bigcup_{i<\alpha}\left\{\bar{a}_{i}, \bar{b}_{i}\right\}$. By simplicity,

$\left({ }^{2}\right)$ The equivalence follows from Corollaries 3.28 and 3.15 . In the $\aleph_{0}$-stable case we always have the Tarski-Vaught property (Remark 3.10). 
$\bar{b} \downarrow_{A \cup \bar{a}_{\alpha}} A \cup \bar{a}_{\alpha}$, and then by Proposition 2.10 we find $\bar{b}_{\alpha}$ such that $\left(\bar{b}, \bar{b}_{\alpha}\right)$ is a beginning of a strongly $\left(A \cup \bar{a}_{\alpha}\right)$-indiscernible sequence and $\bar{b}_{\alpha} \downarrow \downarrow_{A \cup \bar{a}_{\alpha}}$ $\bigcup_{i<\alpha} A \cup\left\{\bar{a}_{\alpha}\right\} \cup\left\{\bar{a}_{i}, \bar{b}_{i}\right\}$. Now both (2) and (3) hold for $i \leq \alpha$.

Let $f \in \operatorname{Aut}(\mathfrak{M} / A \cup \bar{b})$ be such that $f(\bar{a})=\bar{a}_{\alpha}$, and $g \in \operatorname{Aut}\left(\mathfrak{M} / A \cup \bar{a}_{\alpha}\right)$ such that $g(\bar{b})=\bar{b}_{\alpha}$. Now $g \circ f\left(\bar{a}^{\frown} \bar{b}\right)=g\left(\bar{a}_{\alpha} \bar{b}\right)=\bar{a}_{\alpha} \bar{b}_{\alpha}$, and thus (1) holds. Then let $B \subset \bigcup_{i<\alpha}\left\{\bar{a}_{i}, \bar{b}_{i}\right\}$ be finite. By monotonicity, we have $\bar{a}_{\alpha} \downarrow_{A} B$ and $\bar{b}_{\alpha} \downarrow_{A \cup \bar{a}_{\alpha}} B$, and by the finite pairs lemma we get $\bar{a}_{\alpha}^{\curlyvee} \bar{b}_{\alpha} \downarrow_{A} B$. Thus (4) also holds.

Finally, we see that $\bar{b}_{i} \downarrow_{A} \bar{a}_{j}$ if and only if $i>j$. The case $i=j$ follows from (1) and the assumption. Also if $i>j$, from (4) it follows that $\bar{b}_{i} \downarrow_{A} \bar{a}_{j}$. It remains to study the case when $i<j$. Let $0 \leq i<j$. By item $(4), \operatorname{tp}^{\mathrm{w}}\left(\bar{a}_{j} / A \cup\right.$ $\left.\bar{b} \cup \bar{b}_{i}\right)$ does not Lascar-split over $A$. Since $\left(\bar{b}, \bar{b}_{i}\right)$ is a beginning of a strongly $A$-indiscernible sequence, we must have $\operatorname{tp}^{\mathrm{w}}\left(\bar{b} / A \cup \bar{a}_{j}\right)=\operatorname{tp}^{\mathrm{w}}\left(\bar{b}_{i} / A \cup \bar{a}_{j}\right)$. Furthermore by (2), $\bar{b} \downarrow_{A} \bar{a}_{j}$, and hence $\bar{b}_{i} \Varangle_{A} \bar{a}_{j}$.

Finally, we get symmetry as in the $\aleph_{0}$-stable case, with a suitable linear ordering contradicting weak stability.

Proposition 2.20 (Finite symmetry). Let $A$ be finite. Then $\bar{a} \downarrow_{A} \bar{b}$ if and only if $\bar{b} \downarrow_{A} \bar{a}$.

Proof. Assume to the contrary that $\bar{a} \downarrow_{A} \bar{b}$ but $\bar{b} \downarrow_{A} \bar{a}$ for some $\bar{a}, \bar{b}$ and finite $A$. By the previous proposition, there is a sequence $\left(\bar{a}_{i}, \bar{b}_{i}\right)_{i<\mathrm{H}}$ such that $\bar{b}_{i} \downarrow_{A} \bar{a}_{j}$ if and only if $i>j$. Let $\lambda$ be a cardinal such that $(\mathbb{K}, \preccurlyeq \mathbb{K})$ is stable in $\lambda$. Then let $I$ be a linear ordering such that $|I|>\lambda$, and there is a dense set $I_{0} \subset I$ of size $\lambda$.

By Lemma 2.4, there are $\left(\bar{a}_{i}, \bar{b}_{i}\right)_{i \in I}$ such that $\bar{b}_{i} \downarrow_{A} \bar{a}_{j}$ if and only if $i>j$. But now there are $|I|$-many different types over the set $\left(\bar{b}_{i}, \bar{a}_{i}\right)_{i \in I_{0}}$, a contradiction.

We continue to prove other restricted properties of $\downarrow$.

Lemma 2.21. Let $E$ be finite and $\left.\bar{c} \downarrow_{E} \bar{a}\right\urcorner \bar{b}$. If $\operatorname{Lstp}(\bar{a} / E)=\operatorname{Lstp}(\bar{b} / E)$, then $\operatorname{tp}^{\mathrm{w}}(\bar{a} / E \cup\{\bar{c}\})=\operatorname{tp}^{\mathrm{w}}(\bar{b} / E \cup\{\bar{c}\})$.

Proof. Let $\mathscr{A}$ be an $\omega$-saturated model containing $E \cup\{\bar{a}, \bar{b}\}$. Let $\bar{c}^{\prime}$ be such that $\operatorname{tp}^{\mathrm{w}}\left(\bar{c}^{\prime} / E \cup\{\bar{a}, \bar{b}\}\right)=\operatorname{tp}^{\mathrm{w}}(\bar{c} / E \cup\{\bar{a}, \bar{b}\})$ and $\operatorname{tp}^{\mathrm{w}}\left(\bar{c}^{\prime} / \mathscr{A}\right)$ does not Lascar-split over $E$. Let $f \in \operatorname{Aut}(\mathfrak{M} / E)$ be such that $f\left(\bar{c}^{\prime}\right)=\bar{c}$. Hence, $\operatorname{tp}^{\mathrm{w}}(\bar{c} / f(\mathscr{A}))$ does not Lascar-split over $E$. Since $f(\mathscr{A})$ is an $\omega$-saturated model containing $E \cup\{\bar{a}, \bar{b}\}$, and $\operatorname{Lstp}(\bar{a} / E)=\operatorname{Lstp}(\bar{b} / E)$, we must have $\operatorname{tp}^{\mathrm{w}}(\bar{a} / E \cup\{\bar{c}\})=\operatorname{tp}^{\mathrm{w}}(\bar{b} / E \cup\{\bar{c}\})$ by Proposition 2.16 .

Lemma 2.22. Assume that $E$ is finite. If $\bar{a} \downarrow_{E} \bar{c}, \bar{b} \downarrow_{E} \bar{c}$ and $\operatorname{Lstp}(\bar{a} / E)=$ $\operatorname{Lstp}(\bar{b} / E)$, then $\operatorname{tp}^{\mathrm{w}}(\bar{a} / E \cup\{\bar{c}\})=\operatorname{tp}^{\mathrm{w}}(\bar{b} / E \cup\{\bar{c}\})$. 
Proof. Since $\bar{a} \downarrow_{E} \bar{c}$, from Corollary 2.12 we get some $\bar{a}^{\prime}$ such that

$$
\operatorname{Lstp}\left(\bar{a}^{\prime} / E \cup\{\bar{c}\}\right)=\operatorname{Lstp}(\bar{a} / E \cup\{\bar{c}\})
$$

and $\bar{a}^{\prime} \downarrow_{E}\{\bar{b}, \bar{c}\}$. By the finite pairs lemma (Proposition 2.8) since $\bar{b} \downarrow_{E} \bar{c}$, we must have $\left(\bar{a}^{\prime}\right) \frown \bar{b} \downarrow_{E} \bar{c}$. Then, by symmetry we have $\bar{c} \downarrow_{E}\left\{\bar{b}, \bar{a}^{\prime}\right\}$. But $\operatorname{Lstp}\left(\bar{a}^{\prime} / E\right)=\operatorname{Lstp}(\bar{a} / E)=\operatorname{Lstp}(\bar{b} / E), \operatorname{sotp}^{\mathrm{w}}\left(\bar{a}^{\prime} / E \cup\{\bar{c}\}\right)=\operatorname{tp}^{\mathrm{w}}(\bar{b} / E \cup\{\bar{c}\})$ by the previous lemma. By the choice of $\bar{a}^{\prime}$,

$$
\operatorname{tp}^{\mathrm{w}}(\bar{a} / E \cup\{\bar{c}\})=\operatorname{tp}^{\mathrm{w}}(\bar{b} / E \cup\{\bar{c}\}) .
$$

Proposition 2.23 (Restricted finite character). Let $E$ be a finite set. If $\bar{a}\rfloor_{E} B$, then there is a finite $\bar{b} \in B$ such that $\left.\bar{a}\right\rfloor_{E} \bar{b}$.

Proof. By simplicity, $\bar{a} \downarrow_{E} E$. From Corollary 2.12 we get $\bar{a}^{\prime}$ such that $\operatorname{Lstp}\left(\bar{a}^{\prime} / E\right)=\operatorname{Lstp}(\bar{a} / E)$ and $\bar{a}^{\prime} \downarrow_{E} B$. Now we cannot have $\operatorname{tp}^{\mathrm{w}}\left(\bar{a}^{\prime} / E \cup B\right)=$ $\operatorname{tp}^{\mathrm{w}}(\bar{a} / E \cup B)$, and thus there is some finite $\bar{b} \in B$ such that $\operatorname{tp}^{\mathrm{w}}\left(\bar{a}^{\prime} / E \cup\{\bar{b}\}\right) \neq$ $\operatorname{tp}^{\mathrm{w}}(\bar{a} / E \cup\{\bar{b}\})$. By monotonicity, $\bar{a}^{\prime} \downarrow_{E} \bar{b}$. Thus $\bar{a} \downarrow_{E} \bar{b}$ by Lemma 2.22 .

As a corollary we get the following.

Lemma 2.24 (Pairs Lemma). Let $A \subset B$. Assume that $\bar{a} \downarrow_{A} B$ and $\bar{b} \downarrow_{A \cup\{\bar{a}\}} B \cup\{\bar{a}\}$. Then $\bar{a}^{\frown} \bar{b} \downarrow_{A} B$.

Proof. By Proposition 2.6(3), there is a finite $A^{\prime} \subset A$ such that $\bar{a} \downarrow_{A^{\prime}} B$ and $\bar{b} \downarrow_{A^{\prime} \cup\{\bar{a}\}} B \cup\{\bar{a}\}$. We need to show that $\bar{a}^{\urcorner} \bar{b} \downarrow_{A^{\prime}} B$. But by the finite pairs lemma, $\bar{a}\urcorner \bar{b} \downarrow_{A^{\prime}} B^{\prime}$ for each finite $B^{\prime} \subset B$, and thus the claim follows from restricted finite character.

Also the following proposition is clear by symmetry, monotonicity and restricted finite character.

Proposition 2.25 (Left transitivity). Assume that $A, B$ are finite and $\bar{a} \cup B \downarrow_{A} C$. Then $\bar{a} \downarrow_{A \cup B} C$.

Proposition 2.26 (Transitivity). Let $A \subset B \subset C$. If $\bar{a} \downarrow_{A} B$ and $\bar{a} \downarrow_{B} C$, then $\bar{a} \downarrow_{A} C$.

Proof. By Proposition 2.6(3), there are finite $A^{\prime} \subset A$ and finite $B^{\prime} \subset B$ such that $\bar{a} \downarrow_{A^{\prime}} B$ and $\bar{a} \downarrow_{A^{\prime} \cup B^{\prime}} C$. It is enough to show that $\bar{a} \downarrow_{A^{\prime}} C$. By Proposition 2.23 , it is enough to show that $\bar{a} \downarrow_{A^{\prime}} \bar{c}$ for each finite $\bar{c} \in C$, and by finite symmetry, it is enough to show that $\bar{c} \downarrow_{A^{\prime}} \bar{a}$ for each finite $\bar{c} \in C$. Let $\bar{c} \in C$ be finite. Write $\bar{c}=\bar{b} \frown \bar{c}_{0}$, where $\bar{b} \in B$ and $\bar{c}_{0} \in C \backslash B$. We may assume that $\bar{b}$ contains $B^{\prime}$. Then $\bar{a} \downarrow_{A^{\prime}} \bar{b}$ as well as $\bar{a} \downarrow_{A \cup\{\bar{b}\}} \bar{c}_{0}$, and furthermore $\bar{b} \downarrow_{A^{\prime}} \bar{a}$ and $\bar{c}_{0} \downarrow_{A \cup\{\bar{b}\}} \bar{a}$ by symmetry. Hence, $\bar{c} \downarrow_{A^{\prime}} \bar{a}$ by the pairs lemma. This completes the proof for transitivity.

Proposition 2.27 (Stationarity of Lascar strong types, version 1). Let $E$ be finite and assume that $\bar{a} \downarrow_{E} B, \bar{b} \downarrow_{E} B$ and $\operatorname{Lstp}(\bar{a} / E)=\operatorname{Lstp}(\bar{b} / E)$. Then $\operatorname{tp}^{\mathrm{w}}(\bar{a} / E \cup B)=\operatorname{tp}^{\mathrm{w}}(\bar{b} / E \cup B)$. 
Proof. Otherwise, there is a finite $\bar{c} \in B$ such that $\operatorname{tp}^{\mathrm{w}}(\bar{a} / E \cup \bar{c}) \neq$ $\operatorname{tp}^{\mathrm{w}}(\bar{b} / E \cup \bar{c})$, which contradicts Lemma 2.22 .

Proposition 2.28. Let $A \subset B$ with $B$ finite, and assume that $\operatorname{Lstp}(\bar{a} / A)$ $=\operatorname{Lstp}(\bar{b} / A), \bar{a} \downarrow_{A} B$ and $\bar{b} \downarrow_{A} B$. Then $\operatorname{Lstp}(\bar{a} / B)=\operatorname{Lstp}(\bar{b} / B)$.

Proof. By Proposition 2.12 there is $\bar{c}$ realizing $\operatorname{Lstp}(\bar{a} / B)$ such that

$$
\bar{c} \downarrow_{A} B \cup \bar{a} \cup \bar{b} .
$$

By monotonicity and symmetry, $\bar{a} \downarrow_{B} \bar{c}$, and then by transitivity, $\bar{a} \downarrow_{A} B \cup \bar{c}$. Similarly, $\bar{b} \downarrow_{A} B \cup \bar{c}$. Now by Proposition $2.27, \operatorname{tp}^{\mathrm{w}}(\bar{a} / B \cup \bar{c})=\operatorname{tp}^{\mathrm{w}}(\bar{b} / B \cup \bar{c})$. Since $B \cup \bar{c}$ is finite, there is $f \in \operatorname{Aut}(\mathfrak{M} / B \cup \bar{c})$ mapping $\bar{a}$ to $\bar{b}$. Now $\operatorname{Lstp}(\bar{a} / B)=\operatorname{Lstp}(\bar{c} / B)$ implies $\operatorname{Lstp}(f(\bar{a}) / B)=\operatorname{Lstp}(f(\bar{c}) / B)$, and thus $\operatorname{Lstp}(\bar{a} / B)=\operatorname{Lstp}(\bar{c} / B)=\operatorname{Lstp}(\bar{b} / B)$.

DeFinition 2.29. We say that $\bar{a}$ and $\bar{b}$ have the same weak Lascar strong type over $A$, written $\operatorname{Lstp}^{\mathrm{w}}(\bar{a} / A)=\operatorname{Lstp}^{\mathrm{w}}(\bar{b} / A)$, if $\operatorname{Lstp}(\bar{a} / B)=\operatorname{Lstp}(\bar{b} / B)$ for every finite $B \subset A$.

From the above proposition and monotonicity we get the following.

Proposition 2.30 (Stationarity of Lascar strong types, version 2). Let $E$ be finite and assume that $\bar{a} \downarrow_{E} B, \bar{b} \downarrow_{E} B$ and $\operatorname{Lstp}(\bar{a} / E)=\operatorname{Lstp}(\bar{b} / E)$. Then $\operatorname{Lstp}^{\mathrm{w}}(\bar{a} / E \cup B)=\operatorname{Lstp}^{\mathrm{w}}(\bar{b} / E \cup B)$.

We state a lemma about building Morley-type indiscernible sequences.

Lemma 2.31. Assume that $A$ is finite and $\bar{a}$ a tuple. For any ordinal $\lambda$ there exists a strongly $A$-indiscernible sequence $\left(\bar{a}_{i}\right)_{i<\lambda}$ such that $\bar{a}_{0}=\bar{a}$ and

$$
\bar{a}_{i} \downarrow_{A} \bigcup_{j<i} \bar{a}_{j} \quad \text { for each } i<\lambda .
$$

Proof. By strong indiscernibility and finite character it is enough to find a sequence for $\lambda=\omega$.

Again if $\operatorname{tp}^{\mathrm{w}}(\bar{a} / A)$ is bounded, we can take the trivial sequence by Lemma $2.9(1)$. Assume that $\mathrm{tp}^{\mathrm{w}}(\bar{a} / A)$ is unbounded. Using simplicity and extension we define $\left(\bar{b}_{i}\right)_{i<\mathrm{H}}$ such that each $\bar{b}_{i}$ realizes $\operatorname{tp}^{\mathrm{g}}(\bar{a} / A)$ and

$$
\bar{b}_{i} \downarrow_{A} \bigcup_{j<i} \bar{b}_{j} \quad \text { for each } i<\mathrm{H} \text {. }
$$

By Lemma 2.9(2) these $\bar{b}_{i}$ are distinct and we can use Proposition 2.4 to find strongly $A$-indiscernible sequence $\left(\bar{a}_{i}\right)_{i<\omega}$ such that for each $n<\omega$ there are $i_{0}<\cdots<i_{n}<\mathrm{H}$ such that

$$
\operatorname{tp}^{\mathrm{g}}\left(\bar{a}_{0}, \ldots, \bar{a}_{n} / A\right)=\operatorname{tp}^{\mathrm{g}}\left(\bar{b}_{i_{0}}, \ldots, \bar{b}_{i_{n}} / A\right) .
$$

Hence $\bar{a}_{n} \downarrow_{A} \bigcup_{i<n} \bar{a}_{i}$ for each $n<\omega$. Also $\bar{a}_{0}$ realizes $\operatorname{tp}^{\mathrm{g}}(\bar{a} / A)$ and thus there is an automorphism $f \in \operatorname{Aut}(\mathfrak{M} / A)$ mapping $\bar{a}_{0}$ to $\bar{a}$. We may assume that $\bar{a}_{0}=\bar{a}$. 


\section{SUPERSTABILITY}

We would like to find a notion of superstability which would imply all the usual properties of non-forking for $\downarrow$, especially local character. We will suggest notions of superstability and weak superstability, and discuss the relation between them. We will also assume simplicity and weak stability in at least one cardinal, as we did in the previous section. Note that our notion of superstability uses the fact that $\operatorname{LS}(\mathbb{K})=\aleph_{0}$. We also need simplicity to show that $\aleph_{0}$-stability implies superstability (see Corollary 3.28 ).

Definition 3.1. We say that the class $(\mathbb{K}, \preccurlyeq \mathbb{K})$ is superstable if it is weakly stable and the following holds. Let $\left(A_{n}\right)_{n<\omega}$ be an increasing sequence of finite sets such that $\bigcup_{n<\omega} A_{n}$ is a model, and let $\bar{a}$ be a tuple. Then there is $n<\omega$ such that $\bar{a} \downarrow_{A_{n}} A_{n+1}$.

DeFinition 3.2. We say that the class $\left(\mathbb{K}, \preccurlyeq_{\mathbb{K}}\right)$ is weakly superstable if there is a cardinal $\lambda$ such that $(\mathbb{K}, \preccurlyeq \mathbb{K})$ is weakly stable in all cardinals above $\lambda$.

Lemma 3.3 (Local character for models). Assume that a simple finitary $\left(\mathbb{K}, \preccurlyeq_{\mathbb{K}}\right)$ is superstable. Let $\mathscr{A}$ be a model and $\bar{a}$ a tuple. Then there is a finite $A \subset \mathscr{A}$ such that $\bar{a} \downarrow_{A} \mathscr{A}$.

Proof. Let $\bar{a}$ and $\mathscr{A}$ witness the contrary.

We recall the so called presentation theorem for abstract elementary classes with $\operatorname{LS}(\mathbb{K})=\aleph_{0}$. This is the main tool in general abstract elementary classes introduced by Shelah. There is a vocabulary $\tau^{*}$ with $n$-ary function symbols $F_{n}^{k}$ for $k, n<\omega$ such that for each model $\mathscr{B}$ in $\mathbb{K}$ there is a $\tau^{*}$ structure $\mathscr{B}^{*}$ such that $\mathscr{B}^{*} \uparrow \tau=\mathscr{B}$ and whenever a subset $B \subset \mathscr{B}^{*}$ is closed under the functions $\left(F_{k}^{n}\right)^{\mathscr{B}^{*}}$, then $B \preccurlyeq \mathbb{K} \mathscr{B}$. Let $\mathscr{A}^{*} \in \mathbb{K}^{*}$ be such that $\mathscr{A}^{*} \mid \tau=\mathscr{A}$. Define increasing and finite sets $A_{n} \subset \mathscr{A}, n<\omega$, such that

(1) $\left(F_{m}^{k}\right)^{\mathscr{A}^{*}}\left(\left[A_{n}\right]^{m}\right) \subset A_{n+1}$ for $k, m \leq n$,

(2) $\bar{a} \downarrow_{A_{n}} A_{n+1}$ for all $n<\omega$.

We can take $A_{0}=\emptyset$. Assume we have defined $A_{k}$ for $k \leq n$. By assumption, $\bar{a} \downarrow A_{n} \mathscr{A}$, and by Proposition 2.23 there is a finite $A_{n+1}^{\prime} \subset \mathscr{A}$ such that $\bar{a} \downarrow_{A_{n}} A_{n+1}^{\prime}$. We take

$$
A_{n+1}=A_{n+1}^{\prime} \cup\left\{\left(F_{m}^{k}\right)^{\mathscr{A}^{*}}\left(\left[A_{n}\right]^{m}\right): k, m \leq n\right\} .
$$

Then $\bar{a} \downarrow_{A_{n}} A_{n+1}$ by monotonicity.

Finally, $\bigcup_{n<\omega} A_{n}$ is closed under the functions $\left(F_{m}^{k}\right)^{\mathscr{A}^{*}}$ for $m, k<\omega$, and thus is a model. We get a contradiction with superstability.

Corollary 3.4. Assume that a simple finitary $\left(\mathbb{K}, \preccurlyeq_{\mathbb{K}}\right)$ is superstable. Then it is also weakly superstable. Furthermore, let $\mathrm{L}(\mathbb{K})$ be a cardinal such that there are at most $\mathrm{L}(\mathbb{K})$-many Lascar strong types over any finite set. 
For any $\kappa \geq \mathrm{L}(\mathbb{K})$, there are at most $\kappa$-many weak Lascar strong types over a set of size $\kappa$.

Proof. Assume that $(\mathbb{K}, \preccurlyeq \mathbb{K})$ is superstable. Let $\mathrm{L}(\mathbb{K})$ be the cardinal as above. (By the argument at the beginning of Section 3.4 we know that $\mathrm{L}(\mathbb{K})<\mathrm{H}$.) We show the latter claim and thus $(\mathbb{K}, \preccurlyeq \mathbb{K})$ is weakly stable in each $\kappa \geq \mathrm{L}(\mathbb{K})$.

Let $\left(\bar{a}_{i}\right)_{i<\kappa^{+}}$be finite tuples and $\mathscr{A}$ a model such that $|\mathscr{A}|=\kappa \geq \mathrm{L}(\mathbb{K})$. It is enough to find $i<j<\kappa^{+}$such that $\operatorname{Lstp}^{\mathrm{w}}\left(\bar{a}_{i} / \mathscr{A}\right)=\operatorname{Lstp}^{\mathrm{w}}\left(\bar{a}_{j} / \mathscr{A}\right)$. By local character for models, there are finite $E_{i} \subset \mathscr{A}$ such that

$$
\bar{a}_{i} \downarrow_{E_{i}} \mathscr{A} \quad \text { for each } i<\kappa^{+} \text {. }
$$

Since there are only $\kappa$-many finite subsets of $\mathscr{A}$, we can find a subsequence $\left(\bar{a}_{i_{k}}\right)_{k<\kappa^{+}}$such that $E_{i_{k}}=E$ for some fixed finite $E \subset \mathscr{A}$ for all $k<\kappa^{+}$. There are only $\mathrm{L}(\mathbb{K})$-many different Lascar strong types over $E$, and thus there are $k, k^{\prime}<\kappa^{+}$such that $\operatorname{Lstp}\left(\bar{a}_{i_{k}} / E\right)=\operatorname{Lstp}\left(\bar{a}_{i_{k^{\prime}}} / E\right)$. But now by stationarity of Lascar strong types, $\operatorname{Lstp}^{\mathrm{w}}\left(\bar{a}_{i_{k}} / \mathscr{A}\right)=\operatorname{Lstp}^{\mathrm{w}}\left(\bar{a}_{i_{k^{\prime}}} / \mathscr{A}\right)$.

We collect here the properties of $\downarrow$ that we gain from simplicity and superstability. Since we only have local character for models, these properties are still incomplete: we only have the independence calculus for models and finite sets. We will gain local character and the full independence calculus for all sets in Theorem 3.13, where we also assume the Tarski-Vaught property.

THEOREM 3.5. Assume that $\left(\mathbb{K}, \preccurlyeq_{\mathbb{K}}\right)$ is a simple, superstable, finitary $A E C$. Then the relation $\downarrow$ has the following properties.

(1) Invariance: If $A \downarrow_{C} B$, then $f(A) \downarrow_{f(C)} f(B)$ for any $f \in \operatorname{Aut}(\mathfrak{M})$.

(2) Monotonicity: If $A \downarrow_{B} D$ and $B \subset C \subset D$ then $A \downarrow_{C} D$ and $A \downarrow_{B} C$.

(3) Local character for models: For any finite $\bar{a}$ and any $B$ there exists a finite $E \subset B$ such that $\bar{a} \downarrow_{E} B$.

(4) Transitivity: Let $B \subset C \subset D$. If $A \downarrow_{B} C$ and $A \downarrow_{C} D$, then $A \downarrow_{B} D$.

(5) Restricted finite character: Assume that $C$ is finite. $A \downarrow_{C} B$ if and only if $\bar{a} \downarrow_{C} \bar{b}$ for every finite $\bar{a} \in A$ and $\bar{b} \in B$.

(6) Finite character for models: Assume that $\mathscr{C}$ is a model. $A \downarrow_{\mathscr{C}} B$ if and only if $\bar{a} \downarrow_{\mathscr{C}} \bar{b}$ for every finite $\bar{a} \in A$ and $\bar{b} \in B$.

(7) Reflexivity for finite sets: Assume that $C$ is finite and $\operatorname{tp}^{\mathrm{w}}(\bar{a} / C)$ is not bounded. Then $\bar{a}\rfloor_{C} \bar{a}$.

(8) Reflexivity for models: Assume that $\mathscr{C}$ is a model and $\operatorname{tp}^{\mathrm{w}}(\bar{a} / \mathscr{C})$ is not bounded. Then $\bar{a} \Varangle_{\mathscr{C}} \bar{a}$.

(9) Stationarity: If $\operatorname{Lstp}^{\mathrm{w}}(\bar{a} / C)=\operatorname{Lstp}^{\mathrm{w}}(\bar{b} / C), \bar{a} \downarrow_{C} B$ and $\bar{b} \downarrow_{C} B$, then $\operatorname{Lstp}^{\mathrm{w}}(\bar{a} / B \cup C)=\operatorname{Lstp}^{\mathrm{w}}(\bar{b} / B \cup C)$. 
(10) Extension for finite sets: For any finite $C, \bar{a}$ and any $B$ containing $C$, there is $\bar{b}$ such that $\operatorname{Lstp}(\bar{b} / C)=\operatorname{Lstp}(\bar{a} / C)$ and $\bar{b} \downarrow_{C} B$.

(11) Extension for models: For any $\bar{a}, \mathscr{C}$ a model and $B$ containing $\mathscr{C}$, there is $\bar{b}$ such that $\operatorname{Lstp}^{\mathrm{w}}(\bar{b} / \mathscr{C})=\operatorname{Lstp}^{\mathrm{w}}(\bar{a} / \mathscr{C})$ and $\bar{b} \downarrow_{\mathscr{C}} B$.

(12) Restricted symmetry: Assume that $C$ is finite. $A \downarrow_{C} B$ if and only if $B \downarrow_{C} A$.

(13) Symmetry over models: Assume that $\mathscr{C}$ is a model. $A \downarrow_{\mathscr{C}} B$ if and only if $B \downarrow_{\mathscr{C}} A$.

Proof. Items (1) and (2) were studied in Proposition 2.6, and local character for models was proved in Lemma 3.3. Transitivity was stated in Proposition 2.26 and restricted finite character in Proposition 2.23. The other direction of finite character for models follows from monotonicity. Assume that $\mathscr{C}$ is a model and that $A \downarrow_{\mathscr{C}} B$. By definition, there is $\bar{a} \in A$ such that $\bar{a}\rfloor_{\mathscr{C}} B$. By local character for models, we can choose finite $E \subset \mathscr{C}$ such that $\bar{a} \downarrow_{E} \mathscr{C}$. Let $\bar{b} \in \mathscr{C} \cup B$ be finite. We have $E \subset \mathscr{C} \subset \mathscr{C} \cup\{\bar{b}\}, \bar{a} \downarrow_{E} \mathscr{C}$ and $\bar{a} \downarrow_{\mathscr{C}} \bar{b}$. Hence $\bar{a} \downarrow_{E} \bar{b}$ by transitivity. Since $\bar{b}$ was arbitrary, we have $\bar{a} \downarrow_{E} \mathscr{C} \cup B$ by restricted finite character, a contradiction. This proves finite character for models.

Reflexivity for finite sets is Lemma 2.9(b). For models, assume the contrary. Let $\operatorname{tp}^{\mathrm{w}}(\bar{a} / \mathscr{C})$ be unbounded such that $\mathscr{C}$ is a model and $\bar{a} \downarrow_{\mathscr{C}} \bar{a}$. By local character for models there is a finite $E \subset \mathscr{C}$ such that $\bar{a} \downarrow_{E} \mathscr{C}$. By transitivity and monotonicity, $\bar{a} \downarrow_{E} \bar{a}$. Since the type $\operatorname{tp}^{\mathrm{w}}(\bar{a} / E)$ cannot be bounded, we get a contradiction with (7).

Stationarity follows from Proposition 2.30. Extension for finite sets is Corollary 2.12 with simplicity. We prove extension over models. By local character for models, there is a finite $E \subset \mathscr{C}$ such that $\bar{a} \downarrow_{E} \mathscr{C}$. Then by extension for finite sets there is $\bar{b}$ realizing $\operatorname{Lstp}(\bar{a} / E)$ such that $\bar{b} \downarrow_{E} D$. But now by stationarity, $\bar{b}$ also realizes $\operatorname{Lstp}^{\mathrm{w}}(\bar{a} / \mathscr{C})$. Finite symmetry follows from Proposition 2.20 and restricted finite character. To prove symmetry over models, it is enough to prove that $\bar{a} \downarrow_{\mathscr{C}} \bar{b}$ implies $\bar{b} \downarrow_{\mathscr{C}} \bar{a}$ for each $\bar{a} \in A$ and $\bar{b} \in B$. Then symmetry over models follows by finite character for models. Assume that $\bar{a} \downarrow_{\mathscr{C}} \bar{b}$. By local character for models, there is a finite $E \subset \mathscr{C}$ such that $\bar{a} \downarrow_{E} \mathscr{C}$ and $\bar{b} \downarrow_{E} \mathscr{C}$. Then also $\bar{a} \downarrow_{E} \mathscr{C} \cup \bar{b}$ by transitivity. Let $\bar{c} \in \mathscr{C}$ be an arbitrary finite tuple. We get $\bar{c} \downarrow_{E} \bar{b}$ by symmetry and since $\bar{a} \downarrow_{E}\{\bar{c}, \bar{b}\}$, the pairs lemma implies that $\left.\bar{c}\right\urcorner \bar{a} \downarrow_{E} \bar{b}$. Hence by symmetry again, we have $\bar{b} \downarrow_{E}\{\bar{a}, \bar{c}\}$ for each finite $\bar{c} \in \mathscr{C}$. This implies $\bar{b} \downarrow_{E} \mathscr{C} \cup\{\bar{a}\}$ by restricted finite character, and thus $\bar{b} \downarrow_{\mathscr{C}} \bar{a}$.

We recall the following lemma from [11]. The proof uses finite character of $(\mathbb{K}, \preccurlyeq \mathbb{K})$, and this is the first place in this paper where we really use it. Without finite character we should assume that each $A_{n}$ is a model. This lemma is needed in several places where we deal with models built from finite 
sets, in particular in the tree constructions in Lemma 3.7 and Proposition 3.26 , and in the essential Proposition 3.11, which we use to build primary models.

Lemma 3.6. Assume that $(\mathbb{K}, \preccurlyeq \mathbb{K})$ is a finitary AEC. Let $\left(A_{n}: n<\omega\right)$ be an increasing sequence of sets such that $\bigcup_{n<\omega} A_{n}$ is a model in $\mathbb{K}$. Let $\left(\bar{b}_{n}\right)_{n<\omega}$ be a sequence of finite tuples of the same length such that

$$
\operatorname{tp}^{\mathrm{g}}\left(\bar{b}_{m} / A_{n}\right)=\operatorname{tp}^{\mathrm{g}}\left(\bar{b}_{n} / A_{n}\right) \quad \text { for each } n<m<\omega .
$$

Then there exists a tuple $\bar{a}$ such that

$$
\operatorname{tp}^{\mathrm{g}}\left(\bar{a} / A_{n}\right)=\operatorname{tp}^{\mathrm{g}}\left(\bar{b}_{n} / A_{n}\right) \quad \text { for each } n<\omega .
$$

We give a sufficient condition for $(\mathbb{K}, \preccurlyeq \mathbb{K})$ being superstable. We will also see in Theorem 3.38 that this condition is implied by a very weak version of categoricity. Finite character is needed here, since we work with finite sets, not models.

Lemma 3.7. Let $(\mathbb{K}, \preccurlyeq \mathbb{K})$ be a simple finitary AEC. Assume that there are infinite cardinals $\kappa$ and $\lambda$ such that $\kappa^{\aleph_{0}} \leq \lambda, \lambda^{\aleph_{0}}>\lambda,(\mathbb{K}, \preccurlyeq \mathbb{K})$ is weakly stable in $\lambda$ and the following holds for $\kappa$. For all $\bar{a}$ and finite $A$ there is a strongly indiscernible sequence $\left(\bar{a}_{i}\right)_{i<\kappa}$ such that for any $\bar{b}$ the set $\{i<\kappa$ : $\left.\bar{b} \downarrow_{A} \bar{a}_{i}\right\}$ has size strictly smaller than $\kappa$. Then $(\mathbb{K}, \preccurlyeq \mathbb{K})$ is superstable.

Proof. Assume to the contrary that $A_{n}$ are increasing and finite, $\bigcup_{n<\omega} A_{n}$ is a model and $\bar{a}\rfloor_{n} A_{n+1}$ for each $n<\omega$.

We define sets $A_{\eta\lceil n}^{k}$ and tuples $\bar{a}_{\eta\lceil n}$ for all $\eta: \omega \rightarrow \lambda$ and $n \leq k<\omega$ such that

(1) $A_{\eta\lceil n}^{k} \subset A_{\eta\lceil n}^{k+1}$ are finite and the type $\operatorname{tp}^{\mathrm{w}}\left(A_{\eta \uparrow n}^{k+1} / A_{\eta\lceil n}^{k}\right)$ is unbounded,

(2) $\bar{a}_{\eta \uparrow 0}=\bar{a}$ and $A_{\eta\lceil 0}^{k}=A_{k}$ for all $k<\omega$,

(3) $\operatorname{tp}^{\mathrm{g}}\left(\bar{a}_{\eta\lceil n+1} / A_{\eta\lceil n}^{n}\right)=\operatorname{tp}^{\mathrm{g}}\left(\bar{a}_{\eta\lceil n} / A_{\eta\lceil n}^{n}\right)$ and $\bar{a}_{\eta\lceil n+1} \downarrow_{A_{\eta\lceil n+1}^{k}} A_{\eta\lceil n+1}^{k+1}$,

(4) $A_{\eta\lceil n+1}^{n}=A_{\eta\lceil n}^{n}$ and the sequence $\left(A_{\eta\lceil n+1}^{n+1}\right)_{\eta(n)<\lambda}$ is strongly $A_{\eta\left\lceil n^{-}\right.}^{n}$ indiscernible,

(5) for all $\bar{b}$, we have $\left.\mid\{\eta(n)<\lambda: \bar{b}\rfloor_{\eta\lceil n}^{n} A_{\eta\lceil n+1}^{n+1}\right\} \mid<\kappa$.

First define $\bar{a}_{\eta\lceil 0}$ and $A_{\eta\lceil 0}^{k}$ as in (2). Assume we have defined $\bar{a}_{\eta\lceil m}$ and $A_{\eta\lceil m}^{k}$ for all $\eta: \omega \rightarrow \lambda$ and $m \leq k<\omega$ for $m \leq n$. Let $\left(\bar{b}_{i}\right)_{i<\kappa}$ be the $A_{\eta\left\lceil n^{-}\right.}^{n}$ indiscernible sequence implied by the assumption, such that $\bar{b}_{0}=A_{\eta \Uparrow n}^{n+1}$. We can stretch this sequence to $\left(\bar{b}_{i}\right)_{i<\lambda}$, and still, for any $\bar{b}$, the set

$$
\left\{i<\lambda: \bar{b} \downarrow_{\eta \uparrow n}^{n} \bar{b}_{i}\right\}
$$

is of size strictly less than $\kappa$. There is an automorphism $f_{i}^{n} \in \operatorname{Aut}\left(\mathfrak{M} / A_{\eta\lceil n}^{n}\right)$ mapping $A_{\eta \mid n}^{n+1}$ to $\bar{b}_{i}$ for each $i<\lambda$, and we can take $f_{0}^{n}=\operatorname{Id}_{\mathfrak{M}}$. When 
$\eta(n)=i$, we define

$$
\begin{aligned}
& A_{\eta\lceil n+1}^{n}=A_{\eta\lceil n}^{n}, \\
& A_{\eta\lceil n+1}^{k}=f_{i}^{n}\left(A_{\eta\lceil n}^{k}\right) \quad \text { for each } n<k<\omega, \\
& \bar{a}_{\eta\lceil n+1}=f_{i}^{n}\left(\bar{a}_{\eta\lceil n}\right) .
\end{aligned}
$$

This completes the construction.

For $\eta: \omega \rightarrow \lambda$ and $n<\omega$, write $g_{\eta}^{n}=f_{\eta(n)}^{n} \circ \cdots \circ f_{\eta(1)}^{1} \circ f_{\eta(0)}^{0} \in \operatorname{Aut}(\mathfrak{M})$, where the automorphisms $f_{i}^{n}$ are as in the previous construction. Always $g_{\eta}^{n+1}\left\lceil A_{n}=g_{\eta}^{n} \uparrow A_{n}\right.$. By finite character of $(\mathbb{K}, \preccurlyeq \mathbb{K})$, the set

$$
\bigcup_{n<\omega} A_{\eta\lceil n}^{n}=\bigcup_{n<\omega} g_{\eta}^{n}\left(A_{n}\right)
$$

is a model. Using Lemma 3.6 and (3), we find $\bar{a}_{\eta}$ for each $\eta: \omega \rightarrow \lambda$ such that

$$
\operatorname{tp}^{\mathrm{g}}\left(\bar{a}_{\eta} / A_{\eta\lceil n}^{n}\right)=\operatorname{tp}^{\mathrm{g}}\left(\bar{a}_{\eta\lceil n} / A_{\eta\lceil n}^{n}\right) \quad \text { for each } n<\omega .
$$

We look at the types of the tuples $\bar{a}_{\eta}$ over the set

$$
B=\bigcup_{\eta: \omega \rightarrow \lambda, n, k<\omega} A_{\eta\lceil n}^{k},
$$

which has size $\lambda$. First we claim that for a fixed $\eta: \omega \rightarrow \lambda$, there are fewer than $\kappa^{\aleph_{0}}$-many $\bar{a}_{\eta^{\prime}}$ realizing $\operatorname{tp}^{\mathrm{w}}\left(\bar{a}_{\eta} / B\right)$.

We prove the claim by pruning the tree of $\eta^{\prime}$ 's at one level $n<\omega$ at a time leaving out all the branches $\eta^{\prime}$ such that $\bar{a}_{\eta^{\prime}}$ cannot realize $\operatorname{tp}^{\mathrm{w}}\left(\bar{a}_{\eta} / B\right)$ for a simple reason. We leave at most $\kappa^{n}$ branches at each level $n$, and the final tree will be of size at most $\kappa^{\aleph_{0}}$. At level 0 there is only one branch $\eta\lceil 0$. Assume that at level $n<\omega$ there remain at most $\kappa^{n}$ branches $\eta^{\prime}\lceil n: n \rightarrow \lambda$. Let $\eta^{\prime}\left\lceil n\right.$ be one such branch with possible extensions $\eta^{\prime}(n)<\lambda$. If $\bar{a}_{\eta}$ realizes $\operatorname{tp}^{\mathrm{w}}\left(\bar{a}_{\eta^{\prime}\lceil n+1} / A_{\eta^{\prime}\lceil n+1}^{n+1}\right)$, we must have $\bar{a}_{\eta} \downarrow A_{\eta^{\prime}\lceil n+1}^{n} A_{\eta^{\prime}\lceil n+1}^{n+1}$ by (3). But by (5), this can only happen for at most $\kappa$-many $\eta^{\prime}(n)<\lambda$. We leave only those extensions to the tree. We do the pruning for each branch $\eta^{\prime}\lceil n$ of the tree and are left with at most $\kappa^{n+1}$-many branches $\eta^{\prime}\lceil n+1$ at level $n+1$. This proves the claim.

Let us partition the tuples $\bar{a}_{\eta}$ into equivalence classes according to their weak types over the set $B$. Now by $\lambda$-stability, there are at most $\lambda$-many classes, and by the previous claim, each class is of size at most $\kappa^{\aleph_{0}}$. This is a contradiction, since the number of tuples is $\lambda^{\aleph_{0}}>\lambda \times \kappa^{\aleph_{0}}$.

3.1. Tarski-Vaught property. In [12] we used finite character and $\aleph_{0}$-stability to construct models. These properties imply that whenever a set $A$ has the property that each Galois type over each finite subset of $A$ is satisfied in $A$, then $A$ is actually a model. Since in this case there are 
only countably many Galois types over each finite set, we had a useful tool for extending an arbitrary set $A$ to a model of size $|A|+\aleph_{0}$. Here we need a similar property. The finite character property generalizes the idea that $\preccurlyeq \mathbb{K}$ would be induced by a language with finitely many free variables in each formula. Respectively the Tarski-Vaught property can be seen as a generalization of the "countable" Tarski-Vaught criterion for elementary classes: To check whether a set is an elementary submodel, it is enough to see that it is existentially closed with respect to all formulas in the countable language. We use sets of Galois types over the empty set to generalize the notion of a formula in a language.

Definition 3.8. We define an abstract $n$-formula $\phi$ to be a set of Galois types $\operatorname{tp}^{\mathrm{g}}(\bar{a} / \emptyset)$ of $n$-tuples $\bar{a}$ over the empty set. For an $n$-tuple $\bar{b} \in \mathscr{B}$, write $\mathscr{B} \models \phi(\bar{b})$ if $\operatorname{tp}^{\mathrm{g}}(\bar{b} / \emptyset, \mathscr{B}) \in \phi$.

Assumption 3.9 (Tarski-Vaught property). Let $\mathbf{S}$ be a set of abstract formulas. We say that a set $A \subset \mathfrak{M}$ is $\mathbf{S}$-saturated if the following holds. For any finite $\bar{a} \in A, \bar{b} \in \mathfrak{M}$ and $\phi \in \mathbf{S}$, if $\mathfrak{M}=\phi(\bar{a} \bar{b})$, then there is $\bar{d} \in A$ such that $\mathfrak{M} \models \phi(\bar{a} \bar{d})$.

We define the Tarski-Vaught property to be the following: There is a countable set $\mathbf{S}$ of abstract formulas such that any $\mathbf{S}$-saturated subset $A \subset \mathfrak{M}$ is a $\mathbb{K}$-elementary submodel of $\mathfrak{M}$.

Remark 3.10. Assume that $(\mathbb{K}, \preccurlyeq \mathbb{K})$ is an $\aleph_{0}$-stable finitary AEC. Then it has the Tarski-Vaught property.

Proof. We can take as $\mathbf{S}$ the set of all singletons of Galois types over the empty set. By $\aleph_{0}$-stability, there are only countably many of them. By finite character, any $\aleph_{0}$-saturated subset is a model (Lemma 3.8 of [11]).

The following useful proposition uses finite character of $(\mathbb{K}, \preccurlyeq \mathbb{K})$ in the form of Lemma 3.6.

Proposition 3.11. Assume that a finitary $(\mathbb{K}, \preccurlyeq \mathbb{K})$ is simple, superstable and has the Tarski-Vaught property. Let $\left(A_{i}\right)_{i<\omega}$ be finite and increasing and let $\left(\bar{a}_{i}\right)_{i<\omega}$ be tuples such that for $i<j, \operatorname{Lstp}\left(\bar{a}_{j} / A_{i}\right)=\operatorname{Lstp}\left(\bar{a}_{i} / A_{i}\right)$. Then there is some $i<\omega$ such that $\bar{a}_{i+1} \downarrow_{A_{i}} A_{i+1}$.

Proof. We define tuples $\bar{b}_{i}$ and finite increasing sets $B_{i}$ as follows:

(1) $B_{i}=A_{i} \cup \bigcup_{j<i} \bar{b}_{j}$ for each $i<\omega$.

(2) $\bar{b}_{i} \downarrow_{B_{i}}\left(\bigcup_{j<\omega} \bar{a}_{j} \cup \bigcup_{j<\omega} A_{j}\right)$ for each $i<\omega$.

(3) $B=\bigcup_{i<\omega} B_{i}$ satisfies the following: For each finite $\bar{b} \in B, \bar{d} \in \mathfrak{M}$ and $\phi \in \mathbf{S}$ such that $\mathfrak{M} \models \phi(\bar{b} \bar{d})$ there is $\bar{c} \in B$ such that $\mathfrak{M}=\phi(\bar{b} \bar{c})$.

When we have defined $B_{n}$, let $\left(\bar{c}_{j}^{n}\right)_{j<\omega}$ be tuples such that whenever there exists a tuple $\bar{c}$ such that $\mathfrak{M} \models \phi(\bar{b}, \bar{c})$ for some $\phi \in \mathbf{S}$ and finite $\bar{b} \in B_{n}$, 
then one such $\bar{c}$ is listed as $\bar{c}_{j}^{n}$ for some $j<\omega$. Then let $\bar{d}_{n}$ contain $\bar{c}_{j}^{n^{\prime}}$ for every $j, n^{\prime} \leq n$. If each $\operatorname{tp}^{\mathrm{g}}\left(\bar{d}_{n} / B_{n}\right)$ is realized in $B$, then clearly (3) holds.

First let $B_{0}=A_{0}$. Assume we have defined $B_{n}$ and $\bar{b}_{i}$ for each $i<n$. Since $\bar{d}_{n} \downarrow_{B_{n}} B_{n}$ by simplicity, we can use extension to get $\bar{b}_{n}$ such that $\operatorname{tp}^{\mathrm{g}}\left(\bar{b}_{n} / B_{n}\right)=\operatorname{tp}^{\mathrm{g}}\left(\bar{d}_{n} / B_{n}\right)$ and $(2)$ holds for $n$. Then let $B_{n+1}=A_{n} \cup \bigcup_{i<n} \bar{b}_{i}$. This completes the construction.

We claim that when $i, j \geq n$,

$$
\bar{a}_{i} \downarrow_{A_{j}} B_{n} .
$$

We prove the claim by induction on $n$ and for all $i, j \geq n$ simultaneously. By simplicity, $\bar{a}_{i} \downarrow_{A_{j}} A_{j}$ for each $i, j \geq n$. Since $B_{0} \subset A_{j}$ for each $j$, this implies the claim for $n=0$. Assume we have shown the claim for $n$ and let $i, j \geq n+1$. By $(2), \bar{b}_{n} \downarrow_{B_{n}} \bar{a}_{i} \cup A_{j}$, and thus by monotonicity and finite symmetry, $\bar{a}_{i} \downarrow_{B_{n} \cup A_{j}} \bar{b}_{n}$. Induction and finite transitivity imply that $\bar{a}_{i} \downarrow_{A_{j}} B_{n} \cup \bar{b}_{n}$. Since $B_{n+1}=B_{n} \cup \bar{b}_{n} \cup A_{n+1} \subset B_{n} \cup \bar{b}_{n} \cup A_{j}$, this gives the claim.

Now we have $\operatorname{Lstp}\left(\bar{a}_{j} / A_{n}\right)=\operatorname{Lstp}\left(\bar{a}_{n} / A_{n}\right), \bar{a}_{n} \downarrow_{A_{n}} B_{n}$ and $\bar{a}_{j} \downarrow_{A_{n}} B_{n}$ for each $n<j<\omega$, and thus by stationarity,

$$
\operatorname{tp}^{\mathrm{g}}\left(\bar{a}_{j} / B_{n}\right)=\operatorname{tp}^{\mathrm{g}}\left(\bar{a}_{n} / B_{n}\right) \quad \text { for each } n<j<\omega .
$$

Since $B=\bigcup_{n<\omega} B_{n}$ is a model by (3), we can use Lemma 3.6 to get a tuple $\bar{a}$ such that

$$
\operatorname{tp}^{\mathrm{g}}\left(\bar{a} / B_{n}\right)=\operatorname{tp}^{\mathrm{g}}\left(\bar{a}_{n} / B_{n}\right) \quad \text { for each } n<\omega .
$$

Since $B$ is a model, by superstability there is $n<\omega$ such that $\bar{a} \downarrow_{B_{n}} B_{n+1}$, and furthermore by invariance, $\bar{a}_{n+1} \downarrow_{B_{n}} B_{n+1}$. By the previous claim, $\bar{a}_{n+1} \downarrow_{A_{n}} B_{n}$, and thus by finite transitivity, $\bar{a}_{n+1} \downarrow_{A_{n}} B_{n+1}$. Since $A_{n+1} \subset$ $B_{n+1}$, this $\bar{a}_{n+1}$ is the one required for the proposition.

We can easily derive the following corollary, using the restricted finite character property of $\downarrow$.

Corollary 3.12 (Local character). Let $(\mathbb{K}, \preccurlyeq \mathbb{K})$ be a simple, superstable, finitary AEC with the Tarski-Vaught property. Assume that $\bar{a}$ is a tuple and $A$ is an arbitrary set. Then there is a finite $E \subset A$ such that $\bar{a} \downarrow_{E} A$.

Finally, we get the usual properties of non-forking for complete types over arbitrary sets. The proof of the following is analogous to the proof of Theorem 3.5.

THEOREM 3.13. Assume that $\left(\mathbb{K}, \preccurlyeq_{\mathbb{K}}\right)$ is a simple, superstable, finitary AEC with the Tarski-Vaught property. Then the relation $\downarrow$ has the following properties.

(1) Invariance: If $A \downarrow_{C} B$, then $f(A) \downarrow_{f(C)} f(B)$ for any $f \in \operatorname{Aut}(\mathfrak{M})$. 
(2) Monotonicity: If $A \downarrow_{B} D$ and $B \subset C \subset D$ then $A \downarrow_{C} D$ and $A \downarrow_{B} C$.

(3) Local character: For any finite $\bar{a}$ and any $B$ there exists a finite $E \subset B$ such that $\bar{a} \downarrow_{E} B$.

(4) Transitivity: Let $B \subset C \subset D$. If $A \downarrow_{B} C$ and $A \downarrow_{C} D$, then $A \downarrow_{B} D$.

(5) Finite character: $A \downarrow_{C} B$ if and only if $\bar{a} \downarrow_{C} \bar{b}$ for every finite $\bar{a} \in A$ and $\bar{b} \in B$.

(6) Reflexivity: If $\operatorname{tp}^{\mathrm{w}}(\bar{a} / A)$ is not bounded, then $\bar{a} \downarrow_{A} \bar{a}$.

(7) Stationarity: If $\operatorname{Lstp}^{\mathrm{w}}(\bar{a} / C)=\operatorname{Lstp}^{\mathrm{w}}(\bar{b} / C), \bar{a} \downarrow_{C} B$ and $\bar{b} \downarrow_{C} B$, then $\operatorname{Lstp}^{\mathrm{w}}(\bar{a} / B \cup C)=\operatorname{Lstp}^{\mathrm{w}}(\bar{a} / B \cup C)$.

(8) Extension: For any $\bar{a}, C$ and $B$ containing $C$ there is $\bar{b}$ such that $\operatorname{Lstp}^{\mathrm{w}}(\bar{b} / C)=\operatorname{Lstp}^{\mathrm{w}}(\bar{a} / C)$ and $\bar{b} \downarrow_{C} B$.

(9) Symmetry: $A \downarrow_{C} B$ if and only if $B \downarrow_{C} A$.

3.2. Weak Lascar strong type and superstability. In this section we study the behaviour of weak Lascar strong types in superstable simple finitary AECs. First we study when so called abstract weak Lascar strong types are realized. We say that $p$ is an abstract weak Lascar strong type over $A$ if $p$ is a collection

$$
p=\left\{\operatorname{Lstp}\left(\bar{a}_{B} / B\right): B \subset A \text { finite }\right\},
$$

where $B \subset B^{\prime} \subset A$ implies $\operatorname{Lstp}\left(\bar{a}_{B^{\prime}} / B\right)=\operatorname{Lstp}\left(\bar{a}_{B} / B\right)$. For finite $B \subset A$, the type $\operatorname{Lstp}\left(\bar{a}_{B} / B\right) \in p$ may also be denoted as $p\lceil B$. We say that $p$ is realized by $\bar{a}$ if $\operatorname{Lstp}(\bar{a} / B) \in p$ for all finite $B \subset A$. We will show that abstract weak Lascar strong types over models are realized in superstable simple finitary classes, and if in addition the class has the Tarski-Vaught property, then all abstract weak Lascar strong types are realized. For these proofs we need versions of local character for abstract types. When $p$ is an abstract weak Lascar strong type over $A$, we say that $p$ is independent of $A$ over $E$, written

$$
p \downarrow_{E} A,
$$

if $a_{B} \downarrow_{E} B$ for all finite $B \subset A$ such that $E \subset B$ and $\operatorname{Lstp}\left(\bar{a}_{B} / B\right) \in p$.

LEMma 3.14. Assume that $\left(\mathbb{K}, \preccurlyeq_{\mathbb{K}}\right)$ is a simple, superstable finitary AEC. Let $\mathscr{A}$ be a model and $p$ an abstract weak Lascar strong type over $A$. Then there is a finite $E \subset \mathscr{A}$ such that $p \downarrow_{E} \mathscr{A}$.

Proof. Let $\mathscr{A}$ and $p$ witness the contrary. The proof is analogous to the proof of Lemma 3.3. Let again $F_{n}^{k}$ for $k, n<\omega$ be function symbols from the presentation theorem and $\mathscr{A}^{*}$ be the extension of $\mathscr{A}$. Define increasing and finite sets $A_{n} \subset \mathscr{A}$ and tuples $\bar{a}_{n}$ for $n<\omega$ such that

(1) $\operatorname{Lstp}\left(\bar{a}_{n} / A_{n}\right) \in p$ for each $n<\omega$,

(2) $\left(F_{m}^{k}\right)^{\mathscr{A} *}\left(A_{n}\right) \subset A_{n+1}$ for $k, m \leq n$,

(3) $\bar{a}_{n+1} \downarrow_{A_{n}} A_{n+1}$ for each $n<\omega$. 
We can take $A_{0}=\emptyset$ and $\bar{a}_{0}$ realizing $p \nmid \emptyset$. Assume we have defined $A_{k}$ for $k \leq n$. By assumption, $p \nmid A_{n} \mathscr{A}$, and thus there is some finite $A_{n+1}^{\prime} \subset \mathscr{A}$ such that $A_{n} \subset A_{n+1}^{\prime}$ and $\left(p\left\lceil A_{n+1}^{\prime}\right) \downarrow_{A_{n}} A_{n+1}\right.$. Let

$$
A_{n+1}=A_{n+1}^{\prime} \cup\left\{\left(F_{m}^{k}\right)^{\mathscr{A}^{*}}\left(A_{n}\right): k, m \leq n\right\}
$$

and $\bar{a}_{n+1}$ be a tuple realizing $p\left\lceil A_{n+1}\right.$. Since $\bar{a}_{n+1}$ also realizes $p\left\lceil A_{n+1}^{\prime}\right.$, we have $\bar{a}_{n+1} \downarrow_{A_{n}} A_{n+1}^{\prime}$, and thus (3) holds by monotonicity.

Finally, $\bigcup_{n<\omega} A_{n}$ is closed under the functions $\left(F_{m}^{k}\right)^{\mathscr{A}^{*}}$ for $m, k<\omega$, and thus is a model. We can use Lemma 3.6 to find $\bar{a}$ realizing $\operatorname{tp}^{\mathrm{g}}\left(\bar{a}_{n} / A_{n}\right)$ for each $n<\omega$. Now $\bar{a}$ and $\left(A_{n}\right)_{n<\omega}$ contradict superstability.

Assuming the Tarski-Vaught property we can prove a stronger lemma. The proof is analogous to the previous one. We do not need the functions $F_{m}^{k}$ to contradict superstability, but obtain a contradiction with Proposition 3.11 instead.

Lemma 3.15. Assume that $(\mathbb{K}, \preccurlyeq \mathbb{K})$ is a simple, superstable finitary AEC with the Tarski-Vaught property. Let $p$ be an abstract weak Lascar strong type over a set $A$. Then there is a finite $E \subset A$ such that $p \downarrow_{E} A$.

Now we can use extension to prove the following theorem.

TheOREM 3.16. Assume that $\left(\mathbb{K}, \preccurlyeq_{\mathbb{K}}\right)$ is a superstable, simple, finitary $A E C$. Then each abstract weak Lascar strong type over a model is realized. If in addition $\left(\mathbb{K}, \preccurlyeq_{\mathbb{K}}\right)$ has the Tarski-Vaught property, then all abstract weak Lascar strong types are realized.

Proof. We prove the first claim. Then it is clear how to prove the second claim using Lemma 3.15. Let $p$ be an abstract weak Lascar strong type over a model $\mathscr{A}$. By Lemma 3.14, there is a finite $E \subset \mathscr{A}$ such that $p \downarrow_{E} \mathscr{A}$. Let $\bar{b}$ realize $p\left\lceil E\right.$. By simplicity, $\bar{b} \downarrow_{E} E$, and thus by Corollary 2.12, there is $\bar{a}$ realizing $\operatorname{Lstp}(\bar{b} / E)$ such that $\bar{a} \downarrow_{E} \mathscr{A}$. This $\bar{a}$ realizes $p$ by stationarity.

Another consequence of superstability and the Tarski-Vaught property is that weak Lascar strong type is a stronger notion than Galois type over all countable sets. The proof of this theorem is a similar construction to the one in the $\aleph_{0}$-stable case, when we proved that equivalent Galois types imply equivalent weak types over countable models (see [11]). Again we introduce a notion of an isolated type.

Definition 3.17. We say that the Lascar weak strong type $\operatorname{Lstp}^{\mathrm{w}}(\bar{a} \bar{c} / A)$ is isolated over the pair $(\bar{c}, E)$, for some finite $E \subset A$, if for every $\bar{b}$ such that $\operatorname{Lstp}(\bar{b} \bar{c} / E)=\operatorname{Lstp}(\bar{a} \bar{c} / E)$ we have $\operatorname{Lstp}^{\mathrm{w}}(\bar{b} \bar{c} / A)=\operatorname{Lstp}^{\mathrm{w}}(\bar{a} \bar{c} / A)$.

We remark that $\operatorname{Lstp}(\bar{b} \bar{c} / E)=\operatorname{Lstp}(\bar{a} \bar{c} / E)$ does not necessarily imply that $\operatorname{Lstp}(\bar{b} / E \cup \bar{c})=\operatorname{Lstp}(\bar{a} / E \cup \bar{c})$, although the converse holds. Hence the previous notion of isolation is needed for the proof of Theorem 3.19. 
Proposition 3.18. Let $\left(\mathbb{K}, \preccurlyeq_{\mathbb{K}}\right)$ be a superstable simple finitary $A E C$ with the Tarski-Vaught property. For every set $A$, finite $B \subset A$ and tuples $\bar{b}, \bar{c}$, there is $\bar{a}$ and a finite $E \subset A$ such that $\bar{a} \bar{c}$ realizes $\operatorname{Lstp}(\bar{b} \bar{c} / B)$ and $\operatorname{Lstp}^{\mathrm{w}}(\bar{a} \bar{c} / A)$ is isolated over the pair $(\bar{c}, A)$.

Proof. Let $B, A, \bar{b}$ and $\bar{c}$ witness the contrary. We define finite and increasing sets $A_{n} \subset A$ and tuples $\bar{a}_{n}$ for $n<\omega$ such that

(1) $\bar{b}=\bar{a}_{0}$ and $B \subset A_{0}$,

(2) $\operatorname{Lstp}\left(\bar{a}_{n+1} \bar{c} / A_{n}\right)=\operatorname{Lstp}\left(\bar{a}_{n} \bar{c} / A_{n}\right)$,

(3) $\bar{a}_{n+1} \bar{c} \downarrow_{A_{n}} A_{n+1}$

(4) $\bar{c} \downarrow_{A_{0}} A$.

This will contradict Proposition 3.11. The construction runs as follows. First, by local character, there is a finite $E^{\prime} \subset A$ such that $\bar{c} \downarrow_{E^{\prime}} A$. We take $\bar{a}_{0}=\bar{b}$ and $A_{0}=E^{\prime} \cup B$. Assume we have defined $A_{m}$ and $\bar{a}_{m}$ for $m \leq n$.

By Theorem 3.13(7) there is $\bar{d}$ realizing $\operatorname{Lstp}\left(\bar{a}_{n} / A_{n} \cup \bar{c}\right)$ with $\bar{d} \downarrow_{A_{n} \cup \bar{c}} A$. Then $\bar{d} \bar{c}$ realizes $\operatorname{Lstp}\left(\bar{a}_{n} \bar{c} / A_{n}\right)$. Since $\operatorname{Lstp}^{\mathrm{w}}(\bar{d} \bar{c} / A)$ cannot be isolated over the pair $\left(\bar{c}, A_{n}\right)$, there is $\bar{a}_{n+1}$ such that $\operatorname{Lstp}\left(\bar{a}_{n+1} \bar{c} / A_{n}\right)=\operatorname{Lstp}\left(\bar{d} \bar{c} / A_{n}\right)=$ $\operatorname{Lstp}\left(\bar{a}_{n} \bar{c} / A_{n}\right)$ but $\operatorname{Lstp}^{\mathrm{w}}\left(\bar{a}_{n+1} \bar{c} / A\right) \neq \operatorname{Lstp}^{\mathrm{w}}(\bar{d} \bar{c} / A)$.

Now we cannot have $\bar{a}_{n+1} \bar{c} \downarrow_{A_{n}} A$, since otherwise, as $\bar{d} \downarrow_{A_{n} \cup \bar{c}} A$ and $\bar{c} \downarrow_{A_{n}} A$, the pairs lemma implies that $\bar{d} \bar{c} \downarrow_{A_{n}} A$. But then $\operatorname{Lstp}^{\mathrm{w}}(\bar{d} \bar{c} / A)=$ $\operatorname{Lstp}^{\mathrm{w}}\left(\bar{a}_{n+1} \bar{c} / A\right)$ by stationarity, a contradiction. Thus by finite character there is a finite $A_{n+1} \subset A$ such that $A_{n} \subset A_{n+1} \bar{a}_{n+1} \bar{c} \downarrow A_{n} A_{n+1}$. This completes the construction.

TheOREM 3.19. Assume that $\left(\mathbb{K}, \preccurlyeq_{\mathbb{K}}\right)$ is a simple, finitary superstable AEC with the Tarski-Vaught property. Let $A$ be a countable set. Then

$\operatorname{Lstp}^{\mathrm{w}}(\bar{a} / A)=\operatorname{Lstp}^{\mathrm{w}}(\bar{b} / A) \quad$ implies that $\operatorname{tp}^{\mathrm{g}}(\bar{a} / A)=\operatorname{tp}^{\mathrm{g}}(\bar{b} / A)$.

Proof. Enumerate $A=\left\{c_{n}: n<\omega\right\}$. We define sequences $\bar{a}_{n}$ and finite $A_{n} \subset A$ for each $n<\omega$ such that

(1) $\bar{a}=\bar{a}_{0}, \bar{a}_{n}$ is an initial segment of $\bar{a}_{n+1}$ and $c_{n} \in A_{n} \subset A_{n+1} \subset A$,

(2) $\operatorname{Lstp}^{\mathrm{w}}\left(\bar{a}_{n+1} \bar{a}_{n} / A\right)$ is isolated over the pair $\left(\bar{a}_{n}, A\right)$,

(3) $\mathscr{B}=A \cup \bigcup_{n<\omega} \bar{a}_{n}$ is $\mathbf{S}$-saturated for the countable set $\mathbf{S}$ of abstract formulas from the Tarski-Vaught property: for all finite $\bar{b} \in \mathscr{B}$, $\bar{d} \in \mathfrak{M}$ and $\phi \in \mathbf{S}$ such that $\mathfrak{M} \models \phi(\bar{b} \bar{d})$ there is $\bar{c} \in B$ such that $\mathfrak{M} \models \phi(\bar{b} \bar{c})$.

We define simultaneously tuples $\left(\bar{c}_{j}^{n}\right)_{j<\omega}$ and $\bar{d}_{n} \in \mathfrak{M}$.

First let $\bar{a}_{0}=\bar{a}$ and $A_{0}=\left\{c_{0}\right\}$. Assume we have defined $A_{m}, \bar{a}_{m}$ for $m \leq n$. Let $\left(\bar{c}_{j}^{n}\right)_{j<\omega}$ be tuples such that whenever there exists a tuple $\bar{c}$ such that $\mathfrak{M}=\phi(\bar{b}, \bar{c})$ for some $\phi \in \mathbf{S}$ and finite $\bar{b} \in B_{n}$, then one such $\bar{c}$ is listed as $\bar{c}_{j}^{n}$ for some $j<\omega$. Then let $\bar{d}_{n}$ be finite such that $\bar{c}_{j}^{k} \subset \bar{d}_{n}$ for all $k, j \leq n$. 
By Proposition 3.18 there is $\bar{a}^{\prime}$ and finite $A^{\prime} \subset A$ such that

$$
\operatorname{Lstp}\left(\bar{a}^{\prime} \bar{a}_{n} / A_{n}\right)=\operatorname{Lstp}\left(\bar{d}_{n} \bar{a}_{n} / A_{n}\right)
$$

and $\operatorname{Lstp}\left(\bar{a}^{\prime} \bar{a}_{n} / A\right)$ is isolated over the pair $\left(\bar{a}_{n}, A^{\prime}\right)$. Let $A_{n+1}=A_{n} \cup A^{\prime} \cup c_{n+1}$ and $\bar{a}_{n+1}=\bar{a}_{n} \bar{a}^{\prime}$.

This completes the first construction. Now clearly (1) and (2) hold. Also (3) holds by the construction and the fact that having the same Lascar strong type implies having the same Galois type. Thus

$$
\mathscr{B}=A \cup \bigcup_{n<\omega} \bar{a}_{n}=\bigcup_{n<\omega} A_{n} \cup \bigcup_{n<\omega} \bar{a}_{n}
$$

is a model.

Secondly, we construct, by induction on $n<\omega$, tuples $\bar{b}_{n}$ such that

$$
\operatorname{Lstp}^{\mathrm{w}}\left(\bar{b}_{n} \ldots \bar{b}_{0} / A\right)=\operatorname{Lstp}^{\mathrm{w}}\left(\bar{a}_{n} \ldots \bar{a}_{0} / A\right) .
$$

First let $\bar{b}_{0}=\bar{b}$. Then (3.1) holds by assumption. Assume that we have defined $\bar{b}_{m}$ for $m \leq n$. Let $\bar{b}_{n+1}$ be such that $\operatorname{Lstp}\left(\bar{b}_{n+1} \bar{b}_{n} \ldots \bar{b}_{0} / A_{n+1}\right)=$ $\operatorname{Lstp}\left(\bar{a}_{n+1} \bar{a}_{n} \ldots \bar{a}_{0} / A_{n+1}\right)$. We claim that (3.1) holds for $\bar{b}_{n+1}$. If not, there is a finite $B \subset A$ such that

$$
\operatorname{Lstp}\left(\bar{b}_{n+1} \bar{b}_{n} \ldots \bar{b}_{0} / B\right) \neq \operatorname{Lstp}\left(\bar{a}_{n+1} \bar{a}_{n} \ldots \bar{a}_{0} / B\right) .
$$

We may assume that $A_{n+1} \subset B$. By induction,

$$
\operatorname{Lstp}\left(\bar{a}_{n} \ldots \bar{a}_{0} / B\right)=\operatorname{Lstp}\left(\bar{b}_{n} \ldots \bar{b}_{0} / B\right) .
$$

Let $\bar{c}$ be such that

$$
\operatorname{Lstp}\left(\bar{c} \bar{a}_{n} \ldots \bar{a}_{0} / B\right)=\operatorname{Lstp}\left(\bar{b}_{n+1} \bar{b}_{n} \ldots \bar{b}_{0} / B\right),
$$

and hence also

$$
\begin{aligned}
\operatorname{Lstp}\left(\bar{c} \bar{a}_{n} \ldots \bar{a}_{0} / A_{n+1}\right) & =\operatorname{Lstp}\left(\bar{b}_{n+1} \bar{b}_{n} \ldots \bar{b}_{0} / A_{n+1}\right) \\
& =\operatorname{Lstp}\left(\bar{a}_{n+1} \bar{a}_{n} \ldots \bar{a}_{0} / A_{n+1}\right) .
\end{aligned}
$$

But now by isolation, also

$$
\operatorname{Lstp}\left(\bar{c} \bar{a}_{n} \ldots \bar{a}_{0} / B\right)=\operatorname{Lstp}\left(\bar{a}_{n+1} \bar{a}_{n} \ldots \bar{a}_{0} / B\right),
$$

and thus $\operatorname{Lstp}\left(\bar{b}_{n+1} \bar{b}_{n} \ldots \bar{b}_{0} / B\right)=\operatorname{Lstp}\left(\bar{a}_{n+1} \bar{a}_{n} \ldots \bar{a}_{0} / B\right)$, a contradiction. Now the second construction is complete.

For each finite $A_{n} \subset A, \operatorname{Lstp}\left(\bar{b}_{0} \ldots \bar{b}_{n} / A_{n}\right)=\operatorname{Lstp}\left(\bar{a}_{0} \ldots \bar{a}_{n} / A_{n}\right)$ implies that $\operatorname{tp}^{\mathrm{g}}\left(\bar{b}_{0} \ldots \bar{b}_{n} / A_{n}\right)=\operatorname{tp}^{\mathrm{g}}\left(\bar{a}_{0} \ldots \bar{a}_{n} / A_{n}\right)$. There are automorphisms $f_{n}$, for $n<\omega$, witnessing this. By finite character of $(\mathbb{K}, \preccurlyeq \mathbb{K})$, the mapping

$$
\bigcup_{n<\omega} f_{n} \uparrow\left(A_{n} \cup \bar{a}_{0} \ldots \bar{a}_{n}\right): \mathscr{B} \rightarrow \mathfrak{M}
$$

extends to an automorphism $f \in \operatorname{Aut}(\mathfrak{M} / A)$ such that $f(\bar{a})=f\left(\bar{a}_{0}\right)=\bar{b}_{0}=\bar{b}$. This proves the theorem. 
We recall that $(\mathbb{K}, \preccurlyeq \mathbb{K})$ is said to be $\kappa$-tame if for every model $\mathscr{A}$ and tuples $\bar{b}$ and $\bar{a}$ such that

$$
\operatorname{tp}^{\mathrm{g}}(\bar{a} / \mathscr{A}) \neq \operatorname{tp}^{\mathrm{g}}(\bar{b} / \mathscr{A})
$$

there is $\mathscr{B} \preccurlyeq \mathbb{K} \mathscr{A}$ of size $\leq \kappa$ such that

$$
\operatorname{tp}^{\mathrm{g}}(\bar{a} / \mathscr{B}) \neq \operatorname{tp}^{\mathrm{g}}(\bar{b} / \mathscr{B}) .
$$

We say that $\left(\mathbb{K}, \preccurlyeq_{\mathbb{K}}\right)$ is tame is it is $\operatorname{LS}(\mathbb{K})$-tame. Assuming tameness we can generalize the previous result to weak Lascar strong types over models of arbitrary size.

THEOREM 3.20. Assume that $(\mathbb{K}, \preccurlyeq \mathbb{K})$ is a tame, simple, superstable, finitary AEC with the Tarski-Vaught property. If $\mathscr{A}$ is a model, then $\operatorname{Lstp}^{\mathrm{w}}(\bar{a} / \mathscr{A})=\operatorname{Lstp}^{\mathrm{w}}(\bar{b} / \mathscr{A})$ implies that $\operatorname{tp}^{\mathrm{g}}(\bar{a} / \mathscr{A})=\operatorname{tp}^{\mathrm{g}}(\bar{b} / \mathscr{A})$.

Proof. Assume that $\mathscr{A}$ is a model and $\operatorname{Lstp}^{\mathrm{w}}(\bar{a} / \mathscr{A})=\operatorname{Lstp}^{\mathrm{w}}(\bar{b} / \mathscr{A})$. Then also $\operatorname{Lstp}^{\mathrm{w}}(\bar{a} / \mathscr{B})=\operatorname{Lstp}^{\mathrm{w}}(\bar{b} / \mathscr{B})$ for all countable $\mathscr{B} \preccurlyeq \mathbb{K} \mathscr{A}$. Theorem 3.19 implies that $\operatorname{tp}^{\mathrm{g}}(\bar{a} / \mathscr{B})=\operatorname{tp}^{\mathrm{g}}(\bar{b} / \mathscr{B})$ for all countable $\mathscr{B} \preccurlyeq \mathbb{K} \mathscr{A}$. But now by tameness, $\operatorname{tp}^{\mathrm{g}}(\bar{a} / \mathscr{A})=\operatorname{tp}^{\mathrm{g}}(\bar{b} / \mathscr{A})$.

TheOREM 3.21. Assume that $(\mathbb{K}, \preccurlyeq \mathbb{K})$ is a tame, simple, superstable, finitary AEC with the Tarski-Vaught property. If $\mathscr{A}$ is an a-saturated model, then the following are equivalent:

(1) $\operatorname{Lstp}^{\mathrm{w}}(\bar{a} / \mathscr{A})=\operatorname{Lstp}^{\mathrm{w}}(\bar{b} / \mathscr{A})$.

(2) $\operatorname{tp}^{\mathrm{g}}(\bar{a} / \mathscr{A})=\operatorname{tp}^{\mathrm{g}}(\bar{b} / \mathscr{A})$.

(3) $\operatorname{tp}^{\mathrm{w}}(\bar{a} / \mathscr{A})=\operatorname{tp}^{\mathrm{w}}(\bar{b} / \mathscr{A})$.

Proof. By the previous theorem, (1) implies (2). Clearly (2) implies (3). It is enough to prove that equivalent weak types over $\mathscr{A}$ imply equivalent weak Lascar strong types over $\mathscr{A}$. Let $A \subset \mathscr{A}$ be finite. We want to show that $\operatorname{Lstp}(\bar{a} / A)=\operatorname{Lstp}(\bar{b} / A)$. Since $\mathscr{A}$ is a-saturated, there is $\bar{c} \in \mathscr{A}$ realizing $\operatorname{Lstp}(\bar{a} / A)$. Now since $\operatorname{tp}^{\mathrm{w}}(\bar{a} / \mathscr{A})=\operatorname{tp}^{\mathrm{w}}(\bar{b} / \mathscr{A})$, there is $f \in \operatorname{Aut}(\mathfrak{M} / A \cup \bar{c})$ such that $f(\bar{a})=\bar{b}$. Then by invariance $\operatorname{Lstp}(f(\bar{a}) / A)=\operatorname{Lstp}(f(\bar{c}) / A)$, and thus $\operatorname{Lstp}(\bar{b} / A)=\operatorname{Lstp}(\bar{c} / A)=\operatorname{Lstp}(\bar{a} / A)$.

In Section 3.3 we show that $\aleph_{0}$-stability implies superstability in simple finitary classes. Since $\aleph_{0}$-stability and finite character also imply the TarskiVaught property, the above equivalence also holds in simple, tame $\aleph_{0}$-stable finitary classes. There all $\aleph_{0}$-saturated models are moreover a-saturated, and hence have countable a-saturated models. The implication of Theorem 3.19 does not need tameness, and thus holds in $\aleph_{0}$-stable simple finitary classes.

By Corollary 3.4 and Theorem 3.21 we get the following.

TheOREM 3.22. Assume that a simple, tame finitary $(\mathbb{K}, \preccurlyeq \mathbb{K})$ has the Tarski-Vaught property and is superstable. Then it is Galois-stable in each 
cardinal $\mu \geq \mathrm{L}(\mathbb{K})$, where $\mathrm{L}(\mathbb{K})$ is the least upper bound for the number of Lascar strong types over a finite set.

3.3. Characterization of superstability. In this section we study how the concepts of superstability, weak superstability and $\aleph_{0}$-stability are related. We show that a nice behaviour of weak Lascar strong types implies our notions of weak superstability and superstability being equivalent (Corollary 3.27 ) and that simple $\aleph_{0}$-stable finitary classes are superstable (Corollary 3.28). We also characterize superstability with some equivalent conditions in Theorem 3.29.

We define an auxiliary notion of dominating weak Lascar strong types. We say that the class $(\mathbb{K}, \preccurlyeq \mathbb{K})$ has $\lambda$-dominating weak Lascar strong types if for every model $\mathscr{A}$ of size $\leq \lambda$ and tuples $\bar{a}$ and $\bar{b}$, whenever $\operatorname{Lstp}^{\mathrm{w}}(\bar{a} / \mathscr{A})=$ $\operatorname{Lstp}^{\mathrm{w}}(\bar{b} / \mathscr{A})$, then $\operatorname{tp}^{\mathrm{g}}(\bar{a} / \mathscr{A})=\operatorname{tp}^{\mathrm{g}}(\bar{b} / \mathscr{A})$. We say that the class has dominating weak Lascar strong types if it has $\lambda$-dominating weak Lascar strong types for all $\lambda$. Tameness, Tarski-Vaught property and superstability imply dominating weak Lascar strong types in a simple, finitary $(\mathbb{K}, \preccurlyeq \mathbb{K})$ by Theorem 3.20. We will show that also weak superstability and dominating weak Lascar strong types imply superstability.

LEMma 3.23. Assume that a simple finitary $(\mathbb{K}, \preccurlyeq \mathbb{K})$ has $\lambda$-dominating weak Lascar strong types. Assume that $\bar{a} \downarrow_{A} \mathscr{B} \cup A$, where $\mathscr{B}$ is a model, $|\mathscr{B}| \leq \lambda$ and $A$ is a finite set, not necessarily a subset of $\mathscr{B}$. Assume also that $\mathscr{B} \subset C$, where $C$ is a set. Then there is $g \in \operatorname{Aut}(\mathfrak{M} / A \cup \mathscr{B})$ such that $g(\bar{a}) \downarrow_{A} C$.

Proof. By Corollary 2.12 there is $\bar{b}$ realizing $\operatorname{Lstp}(\bar{a} / A)$ such that $\bar{b} \downarrow_{A}$ $A \cup C$. We write the finite set $A$ as a sequence $\bar{a}^{\prime}$. Now by stationarity (Proposition 2.30), $\operatorname{Lstp}^{\mathrm{w}}(\bar{a} / \mathscr{B} \cup A)=\operatorname{Lstp}^{\mathrm{w}}(\bar{b} / \mathscr{B} \cup A)$, and furthermore $\operatorname{Lstp}^{\mathrm{w}}\left(\bar{a}^{\frown} \bar{a}^{\prime} / \mathscr{B}\right)=\operatorname{Lstp}^{\mathrm{w}}\left(\bar{b} \frown \bar{a}^{\prime} / \mathscr{B}\right)$. Then by $\lambda$-dominating weak Lascar strong types, there is $g \in \operatorname{Aut}(\mathfrak{M} / \mathscr{B})$ such that $g\left(\bar{a}^{\frown} \bar{a}^{\prime}\right)=\bar{b}^{\frown} \bar{a}^{\prime}$. Hence $g(\bar{a}) \downarrow_{A} A \cup C$ and $g \in \operatorname{Aut}(\mathscr{A} / \mathscr{B} \cup A)$.

LEMma 3.24. Assume that a simple, finitary, weakly stable $(\mathbb{K}, \preccurlyeq \mathbb{K})$ has $\lambda$-dominating weak Lascar strong types. Let $A \subset \mathscr{B}$, where $A$ is finite and $\mathscr{B}$ is a model of size $\leq \lambda$, and let $\alpha$ be an ordinal. If $\bar{a} \downarrow_{A} \mathscr{B}$, then there is a strongly $\mathscr{B}$-indiscernible sequence $\left(\bar{a}_{i}\right)_{i<\alpha}$ such that $\bar{a}_{0}=\bar{a}$ and

$$
\bar{a}_{i} \downarrow_{A} \mathscr{B} \cup \bigcup_{j<i} \bar{a}_{j} \quad \text { for each } i<\alpha .
$$

Proof. If $\operatorname{tp}^{\mathrm{w}}(\bar{a} / A)$ is bounded, we can take the trivial sequence. Thus we may assume that $\operatorname{tp}^{\mathrm{w}}(\bar{a} / A)$ is unbounded. Using Proposition 2.12 we define 
$\left(\bar{b}_{i}\right)_{i<\mathrm{H}(|\mathscr{B}|)}$ such that each $\bar{b}_{i}$ realizes $\operatorname{Lstp}(\bar{a} / A)$ and

$$
\bar{b}_{i} \downarrow_{A} \mathscr{B} \cup \bigcup_{j<i} \bar{b}_{j} \quad \text { for each } i<\mathrm{H}(|\mathscr{B}|) .
$$

By stationarity of weak Lascar strong types, each $\bar{b}_{i}$ realizes $\operatorname{Lstp}^{\mathrm{w}}(\bar{a} / \mathscr{B})$ and by $\lambda$-dominating weak Lascar strong types, also $\operatorname{tp}^{\mathrm{g}}(\bar{a} / \mathscr{B})$.

For each $i<\mathrm{H}(|\mathscr{B}|)$, the type $\operatorname{tp}^{\mathrm{w}}\left(\bar{b}_{i} / A\right)=\operatorname{tp}^{\mathrm{w}}(\bar{a} / A)$ is unbounded, and thus by Lemma $2.9(2)$ these $\bar{b}_{i}$ are distinct. We can use Lemma 2.4 to find strongly $\mathscr{B}$-indiscernible sequence $\left(\bar{a}_{i}\right)_{i<\alpha}$ such that for each $n<\omega$ and $j_{0}<\cdots<j_{n}<\alpha$ there are $i_{0}<\cdots<i_{n}<\mathrm{H}(|\mathscr{B}|)$ such that

$$
\operatorname{tp}^{\mathrm{g}}\left(\bar{a}_{j_{0}}, \ldots, \bar{a}_{j_{n}} / \mathscr{B}\right)=\operatorname{tp}^{\mathrm{g}}\left(\bar{b}_{i_{0}}, \ldots, \bar{b}_{i_{n}} / \mathscr{B}\right) .
$$

Hence by finite character, $\bar{a}_{i} \downarrow_{A} \mathscr{B} \cup \bigcup_{j<i} \bar{a}_{j}$ for each $n<\omega$.

Now $\bar{a}_{0}$ realizes $\operatorname{tp}^{\mathrm{g}}(\bar{a} / \mathscr{B})$ and thus there is an automorphism $f$ in $\operatorname{Aut}(\mathfrak{M} / \mathscr{B})$ mapping $\bar{a}_{0}$ to $\bar{a}$. We may assume that $\bar{a}_{0}=\bar{a}$.

In the following lemma we again use the finite character of $(\mathbb{K}, \preccurlyeq \mathbb{K})$.

Lemma 3.25. Assume that a simple finitary weakly stable $(\mathbb{K}, \preccurlyeq \mathbb{K})$ has $\lambda$ dominating weak Lascar strong types. Let $\left(A_{k}\right)_{k<\omega}$ be an increasing sequence of finite sets such that $\bigcup_{k<\omega} A_{k}$ is a model, $A_{2} \cup \bar{a} \downarrow_{A_{1}} \mathscr{C}$ and $A_{k+1} \downarrow_{A_{k} \cup \bar{a}} \mathscr{C}$ for $k \geq 2$. Assume also that $\mathscr{C}$ is a model of size $\leq \lambda$ and $\mathscr{C} \subset D$. Then there is an increasing sequence $\left(B_{k}\right)_{k<\omega}$ of finite sets and finite $\bar{b}$ such that

(1) $\operatorname{tp}^{\mathrm{g}}\left(B_{k} \cup \bar{b} / A_{1} \cup \mathscr{C}\right)=\operatorname{tp}^{\mathrm{g}}\left(A_{k} \cup \bar{a} / A_{1} \cup \mathscr{C}\right)$,

(2) $B_{2} \cup \bar{b} \downarrow_{A_{1}} D$,

(3) $B_{k+1} \downarrow_{B_{k} \cup \bar{b}} D$ for $k \geq 2$,

(4) $\bigcup_{k<\omega} B_{k}$ is a model.

Proof. Since $A_{2} \cup \bar{a} \downarrow_{A_{1}} \mathscr{C}$, by Lemma 3.23 there is $g_{2} \in \operatorname{Aut}\left(\mathfrak{M} / A_{1} \cup \mathscr{C}\right)$ such that $g_{2}\left(A_{2} \cup \bar{a}\right) \downarrow_{A_{1}} D$. We let $\bar{b}=g_{2}(\bar{a})$ and $B_{2}=g_{2}\left(A_{2}\right)$. Since $A_{3} \downarrow_{A_{2} \cup \bar{a}} \mathscr{C}$, by invariance also $g_{2}\left(A_{3}\right) \downarrow_{B_{2} \cup \bar{b}} \mathscr{C}$ and again by Lemma 3.23, there is $g \in \operatorname{Aut}\left(\mathfrak{M} / B_{2} \cup \bar{b} \cup \mathscr{C}\right)$ such that $g\left(g_{2}\left(A_{3}\right)\right) \downarrow_{B_{2} \cup \bar{b}} D$. We let $g_{3}=g \circ g_{2}$ and $B_{3}=g_{3}\left(A_{3}\right)$.

Let $k \geq 3$ and assume we have defined $g_{k} \in \operatorname{Aut}\left(\mathfrak{M} / g_{k-1}\left(A_{k-1} \cup \bar{a}\right)\right)$ such that $g_{k-1}(\bar{a})=\bar{b}, g_{k} \uparrow\left(A_{1} \cup \mathscr{C}\right)=\operatorname{Id}\left(A_{1} \cup \mathscr{C}\right)$ and $g_{k}\left(A_{k}\right) \downarrow_{g_{k-1}\left(A_{k-1} \cup \bar{a}\right)} D$. We set $B_{k}=g_{k}\left(A_{k}\right)$.

Since $A_{k+1} \downarrow_{A_{k} \cup \bar{a}} \mathscr{C}$, also $g_{k}\left(A_{k+1}\right) \downarrow_{g_{k}}\left(A_{k} \cup \bar{a}\right) \mathscr{C}$ and thus by Lemma 3.23, there is $g \in \operatorname{Aut}\left(\mathfrak{M} / g_{k}\left(A_{k} \cup \bar{a}\right) \cup \mathscr{C}\right)$ such that $g\left(g_{k}\left(A_{k+1}\right)\right) \downarrow_{g_{k}\left(A_{k} \cup \bar{a}\right)} D$. We let $g_{k+1}=g \circ g_{k}$. Now, if $B_{k+1}=g_{k+1}\left(A_{k+1}\right)$, we have $\operatorname{tp}^{\mathrm{g}}\left(B_{k} \cup \bar{b} / A_{1} \cup \mathscr{C}\right)$ $=\operatorname{tp}^{\mathrm{g}}\left(A_{k} \cup \bar{a} / A_{1} \cup \mathscr{C}\right)$ and $B_{k+1} \downarrow_{B_{k} \cup \bar{b}} D$ when $k \geq 2$.

Finally, the mapping $\bigcup_{k<\omega} g_{k} \uparrow_{A_{k}}: \bigcup_{k<\omega} A_{k} \rightarrow \bigcup_{k<\omega} B_{k}$ preserves Galois types of finite tuples, and thus by finite character of $(\mathbb{K}, \preccurlyeq \mathbb{K})$ it is a $\mathbb{K}$ embedding. We conclude that $\bigcup_{k<\omega} B_{k}$ is a model. 
Proposition 3.26. Assume that $(\mathbb{K}, \preccurlyeq \mathbb{K})$ is a simple, finitary AEC. Assume also that $(\mathbb{K}, \preccurlyeq \mathbb{K})$ is stable in $\lambda$ and has $\lambda$-dominating weak Lascar strong types for some $\lambda$ such that $\lambda^{\aleph_{0}}>\lambda$. Let $\left(A_{k}\right)_{k<\omega}$ be an increasing sequence of finite sets such that $\bigcup_{k<\omega} A_{k}$ is a model and let $\bar{a}$ be a tuple. Then there is $k<\omega$ such that $\bar{a} \downarrow_{A_{k}} A_{k+1}$.

Proof. Assume the contrary. Let $\left(A_{k}\right)_{k<\omega}$ be an increasing sequence of finite sets such that $\bigcup_{k<\omega} A_{k}$ is a model and $\bar{a} \Varangle_{A_{k}} A_{k+1}$ for all $k<\omega$.

For each mapping $\xi: \omega \rightarrow \lambda$ and $k, n<\omega$, we define finite $A_{\xi \backslash n}^{k}, \bar{a}_{\xi \backslash n}$ and a set $\mathscr{A}_{n}$ such that

(1) $\mathscr{A}_{n} \subset \mathscr{A}_{n+1}$ and $\left|\mathscr{A}_{n}\right| \leq \lambda$ for each $n<\omega$,

(2) $\mathscr{A}_{0}=A_{0}$ is finite but $\mathscr{A}_{n}$ is a model for each $0<n<\omega$,

(3) $\bigcup_{\xi: n \rightarrow \lambda} A_{\xi\lceil n}^{n} \subset \mathscr{A}_{n}$,

(4) when $\xi\left\lceil n=\xi^{\prime}\left\lceil n\right.\right.$ and $\xi^{\prime}(n)<\xi(n)$,

$$
\operatorname{tp}^{\mathrm{g}}\left(\bar{a}_{\xi\lceil n+1} / A_{\xi^{\prime}\lceil n+1}^{n+1}\right) \neq \operatorname{tp}^{\mathrm{g}}\left(\bar{a}_{\xi^{\prime}\lceil n+1} / A_{\xi^{\prime}\lceil n+1}^{n+1}\right),
$$

(5) for $m<n<\omega, \operatorname{tp}^{\mathrm{g}}\left(\bar{a}_{\xi \backslash n} / \mathscr{A}_{m}\right)=\operatorname{tp}^{\mathrm{g}}\left(\bar{a}_{\xi \backslash m} / \mathscr{A}_{m}\right)$.

Then by Lemma 3.6, for each $\xi: \omega \rightarrow \lambda$, we will gain $\bar{a}_{\xi}$ satisfying $\operatorname{tp}^{\mathrm{g}}\left(\bar{a}_{\xi\lceil n} / \mathscr{A}_{n}\right)$ for each $n<\omega$. By (4), these $\bar{a}_{\xi}$ will contradict $\lambda$-stability. We carry out the construction maintaining the following three conditions.

(i) We have $A_{\xi\lceil n}^{k} \subset A_{\xi\lceil n}^{k+1}$ for each $k<\omega$ and $\bigcup_{k<\omega} A_{\xi\lceil n}^{k}$ is a model.

(ii) For each $\xi: \omega \rightarrow \lambda$ and $n<\omega$,

(a) $\xi^{\prime}\left\lceil n=\xi\left\lceil n\right.\right.$ and $\xi^{\prime}(n)<\xi(n)$ imply $A_{\xi\lceil n+1}^{n+1} \downarrow A_{\xi\lceil n}^{n} \mathscr{A}_{n} \cup A_{\xi^{\prime}\lceil n+1}^{n+1}$,

(b) $\left(A_{\xi\lceil n+1}^{n+1}\right)_{\xi(n)<\lambda}$ is a strongly $\mathscr{A}_{n}$-indiscernible sequence with

$$
A_{\xi\lceil n+1}^{n+1}=A_{\xi\lceil n}^{n+1} \quad \text { for } \xi(n)=0,
$$

(c) for each $n<\omega, j<\lambda$ there is $F_{j}^{n+1} \in \operatorname{Aut}\left(\mathfrak{M} / \mathscr{A}_{n}\right)$ such that $F_{j}^{n+1}\left(A_{\xi \nmid n}^{n+1}\right)=A_{\xi \nmid n+1}^{n+1}$ for $\xi$ such that $\xi(n)=j$

(d) the model $\mathscr{A}_{n+1}$ is closed under the functions $F_{j}^{n+1}$ and their inverses for $j<\lambda$.

(iii) If $k \geq n$, then $A_{\xi \backslash n}^{k+1} \downarrow_{A_{\xi \backslash n}^{k} \cup \bar{a}_{\xi \backslash n}} \mathscr{A}_{n}, \bar{a}_{\xi\lceil n} \downarrow_{A_{\xi \backslash n}^{k}} A_{\xi\lceil n}^{k+1}$ and

$$
A_{\xi\lceil n}^{n+1} \cup \bar{a}_{\xi\lceil n} \downarrow_{A_{\xi \backslash n}^{n}} \mathscr{A}_{n} .
$$

First let $A_{\xi\lceil 0}^{k}=A_{k}$ for each $k<\omega, \bar{a}_{\xi\lceil 0}=\bar{a}$ and $\mathscr{A}_{0}=A_{0}=A_{\xi\lceil 0}^{0}$. Then (ii) holds trivially, and (i) and (iii) hold by simplicity, monotonicity and the assumption. Also (1)-(5) hold trivially.

Assume we have defined everything for $m \leq n$. Since $A_{\xi \backslash n}^{n+1} \downarrow_{A_{\xi \backslash n}^{n}} \mathscr{A}_{n}$ by (iii), we can use (Proposition 2.31 or) Lemma 3.24 to find a strongly $\mathscr{A}_{n}$-indiscernible $\left(A_{\xi\lceil n+1}^{n+1}\right)_{\xi(n)<\lambda}$ such that $A_{\xi\lceil n+1}^{n+1}=A_{\xi\lceil n}^{n+1}$ for $\xi(n)=0$ and 
(ii)(a) holds. Then define $F_{j}^{n+1}$ and $\mathscr{A}_{n+1}$ as desired, $F_{0}^{n+1}$ being the identity. We require that $\mathscr{A}_{n} \cup \bigcup_{\xi:(n+1) \rightarrow \lambda} A_{\xi\lceil n+1}^{n+1} \subset \mathscr{A}_{n+1},\left|\mathscr{A}_{n+1}\right| \leq \lambda$ and $\mathscr{A}_{n+1}$ is closed under each $F_{j}^{n+1}$ and $\left(F_{j}^{n+1}\right)^{(-1)}$.

By (iii), we have $A_{\xi \backslash n}^{n+2} \downarrow_{A_{\xi \backslash n}^{n+1} \cup \bar{a}_{\xi \backslash n}} \mathscr{A}_{n}$ and $A_{\xi \backslash n}^{n+1} \cup \bar{a}_{\xi \nmid n} \downarrow_{A_{\xi \backslash n}^{n}} \mathscr{A}_{n}$. The pairs lemma and left transitivity imply that

$$
A_{\xi \backslash n}^{n+2} \cup \bar{a}_{\xi\lceil n} \downarrow_{A_{\xi \backslash n}^{n+1}} \mathscr{A}_{n} .
$$

On the other hand, we have $A_{\xi \backslash n}^{k+1} \downarrow_{A_{\xi \backslash n}^{k} \cup \bar{a}_{\xi \backslash n}} \mathscr{A}_{n}$ and $\bar{a}_{\xi \backslash n} \downarrow_{A_{\xi \backslash n}^{k}} A_{\xi \backslash n}^{k+1}$ for each $k \geq n+1$. By Lemma 3.25, there are $\bar{b}$ and finite $B_{k}$ for $n+1 \leq k<\omega$, such that $B_{n+1}=A_{\xi\lceil n}^{n+1}$ and for each $k \geq n+1$,

(b1) $B_{k} \cup \bar{b}$ realizes $\operatorname{tp}^{\mathrm{g}}\left(A_{\xi\lceil n}^{k} \cup \bar{a}_{\xi\lceil n} / A_{\xi\lceil n}^{n+1} \cup \mathscr{A}_{n}\right)$ and thus $\left.\bar{b}\right\rfloor_{B_{k}} B_{k+1}$,

(b2) $B_{n+2} \cup \bar{b} \downarrow_{A_{\xi \backslash n}^{n+1}} \mathscr{A}_{n+1}$,

(b3) $B_{k+1} \downarrow_{B_{k} \cup \bar{b}} \mathscr{A}_{n+1}$ when $k \geq n+2$,

(b4) $B_{k} \subset B_{k+1}$ and $\bigcup_{k<\omega} B_{k}$ is a model.

Since $B_{n+1}=A_{\xi \uparrow n}^{n+1}$, we have

$$
F_{\xi(n)}^{n+1}\left(B_{n+1}\right)=A_{\xi \backslash n+1}^{n+1} .
$$

For each $\xi(n)<\lambda$ and $n+1<k<\omega$, define

$$
\bar{a}_{\xi \nmid n+1}=F_{\xi(n)}^{n+1}(\bar{b}) \quad \text { and } \quad A_{\xi\lceil n+1}^{k}=F_{\xi(n)}^{n+1}\left(B_{k}\right) .
$$

Since each $F_{j}^{n+1}$ maps $\mathscr{A}_{n+1}$ to itself, we see from (b1)-(b3) that (iii) holds for $n+1$. Also by (b4), (i) holds. We check that (1)-(5) hold. Items (1)-(3) hold by the definition of $\mathscr{A}_{n+1}$. Also (5) holds, since $\bar{b}$ realizes $\operatorname{tp}^{\mathrm{g}}\left(\bar{a}_{\xi\lceil n} / \mathscr{A}_{n}\right)$ and $F_{j}^{n+1} \in \operatorname{Aut}\left(\mathfrak{M} / \mathscr{A}_{n}\right)$ for each $j<\lambda$. We claim that (4) holds.

Let $\xi^{\prime}\left\lceil n=\xi\left\lceil n\right.\right.$ and $\xi^{\prime}(n)<\xi(n)$. Since $\bar{a}_{\xi\lceil n+1} \downarrow_{A_{\xi \backslash n+1}^{n+1}} \mathscr{A}_{n+1}$ by (iii) and $A_{\xi^{\prime} \mid n+1}^{n+1} \subset \mathscr{A}_{n+1}$, we find that $\bar{a}_{\xi\lceil n+1} \downarrow_{A_{\xi\lceil n+1}^{n+1}} A_{\xi^{\prime}\lceil n+1}^{n+1}$. Furthermore, $A_{\xi\lceil n+1}^{n+1} \downarrow_{A_{\xi \backslash n}^{n}} A_{\xi^{\prime} \mid n+1}^{n+1}$ by (ii). The pairs lemma and monotonicity imply that

$$
\bar{a}_{\xi \nmid n+1} \downarrow_{A_{\xi \backslash n}^{n}} A_{\xi^{\prime}\lceil n+1}^{n+1} .
$$

On the other hand, $\left.\bar{a}_{\xi\lceil n}\right\rfloor_{\xi\lceil n}^{n} A_{\xi\lceil n}^{n+1}$ by (iii), and thus $\bar{b} \downarrow_{A_{\xi \backslash n}^{n}} A_{\xi\lceil n}^{n+1}$ by (b1). The automorphism $F_{\xi^{\prime}(n)}^{n+1}$ gives

$$
\bar{a}_{\xi^{\prime} \mid n+1} \downarrow A_{\xi \backslash n}^{n} A_{\xi^{\prime}\lceil n+1}^{n+1} .
$$

We conclude that $\operatorname{tp}^{\mathrm{g}}\left(\bar{a}_{\xi\lceil n+1} / A_{\xi^{\prime}\lceil n+1}^{n+1}\right) \neq \operatorname{tp}^{\mathrm{g}}\left(\bar{a}_{\xi^{\prime}\lceil n+1} / A_{\xi^{\prime}\lceil n+1}^{n+1}\right)$.

From the above proposition we get two important corollaries. 
Corollary 3.27. Assume that $(\mathbb{K}, \preccurlyeq \mathbb{K})$ is a simple finitary $A E C$ with dominating weak Lascar strong types. Then $(\mathbb{K}, \preccurlyeq \mathbb{K})$ is superstable if and only if it is weakly superstable.

Proof. The "only if" direction is Corollary 3.4. If $(\mathbb{K}, \preccurlyeq \mathbb{K})$ is weakly superstable and has dominating weak Lascar strong types, we can clearly find the $\lambda$ required for Proposition 3.26.

Corollary 3.28. Assume that $(\mathbb{K}, \preccurlyeq \mathbb{K})$ is an $\aleph_{0}$-stable simple finitary $A E C$. Then it is superstable.

Proof. By Theorem 3.12 of [11], in $\aleph_{0}$-stable finitary AECs, equivalence of weak types implies equivalence of Galois types over countable models. Thus any $\aleph_{0}$-stable finitary AEC has $\aleph_{0}$-dominating weak Lascar strong types. Superstability follows by Proposition 3.26.

Finally, we give a list of properties equivalent to superstability.

THEOREM 3.29 (Characterization of superstability). Let ( $\mathbb{K}, \preccurlyeq \mathbb{K}$ ) be a simple finitary AEC with the Tarski-Vaught property. The following are equivalent.

(1) The class $(\mathbb{K}, \preccurlyeq \mathbb{K})$ is weakly stable and for all finite and increasing $A_{n}, n<\omega$, and all $\bar{a}$ there is $n<\omega$ such that $\bar{a} \downarrow_{A_{n}} A_{n+1}$.

(2) Superstability: The class $(\mathbb{K}, \preccurlyeq \mathbb{K})$ is weakly stable and for all finite and increasing $A_{n}, n<\omega$, such that $\bigcup_{n<\omega} A_{n}$ is a model and all $\bar{a}$ there is $n<\omega$ such that $\bar{a} \downarrow_{A_{n}} A_{n+1}$.

(3) The class $(\mathbb{K}, \preccurlyeq \mathbb{K})$ is weakly superstable and there is an infinite cardinal $\kappa$ such that for any $\bar{a}$ and finite $A$, there is a strongly $A$ indiscernible $\left(\bar{a}_{i}\right)_{i<\kappa}$ such that for any $\bar{b}$,

$$
\left|\left\{i<\kappa: \bar{b} \downarrow_{A} \bar{a}_{i}\right\}\right|<\kappa .
$$

(4) There are infinite cardinals $\lambda$ and $\kappa$ such that $\kappa^{\aleph_{0}} \leq \lambda, \lambda^{\aleph_{0}}>\lambda$, $(\mathbb{K}, \preccurlyeq \mathbb{K})$ is weakly stable in $\lambda$ and for any $\bar{a}$ and finite $A$, there is a strongly $A$-indiscernible $\left(\bar{a}_{i}\right)_{i<\kappa}$ such that for any $\bar{b}$,

$$
\left|\left\{i<\kappa: \bar{b} \downarrow_{A} \bar{a}_{i}\right\}\right|<\kappa .
$$

If the class is also tame, (1)-(4) are equivalent to

(5) The class $(\mathbb{K}, \preccurlyeq \mathbb{K})$ is weakly superstable and whenever a finite tuple $\bar{b}$ realizes $\operatorname{Lstp}^{\mathrm{w}}(\bar{a} / \mathscr{A})$, where $\mathscr{A}$ is a model, there is $f \in \operatorname{Aut}(\mathfrak{M} / \mathscr{A})$ such that $f(\bar{b})=\bar{a}$.

Proof. Items (1) and (2) are equivalent by Proposition 3.11. By Lemma 3.7, (4) implies (2). Clearly also (3) implies (4). We show that (1) implies (3), which completes the proof of the first part of the theorem. By Corollary 3.4, (1) implies weak superstability. To prove (3), let $\bar{a}$ and $A$ be finite. We 
prove (3) for the infinite cardinal $\kappa=\aleph_{0}$. By Lemma 2.31, there is a strongly $A$-indiscernible sequence $\left(\bar{a}_{n}\right)_{n<\omega}$ such that $\bar{a}_{0}=\bar{a}$ and

$$
\bar{a}_{n} \downarrow_{A} \bigcup_{m<n} \bar{a}_{m} \quad \text { for each } n<\omega .
$$

We claim that this is the required sequence. We assume the contrary, that there would be some $\bar{b}$ such that $\bar{b} \downarrow_{A} \bar{a}_{n}$ for infinitely many $n<\omega$. Let $\left(\bar{b}_{n}\right)_{n<\omega}$ be this infinite subsequence. Then we claim that

$$
\bar{b} \downarrow_{A \cup \cup_{m<n} \bar{b}_{m}} \bar{b}_{n} \quad \text { for each } n<\omega .
$$

To prove this second claim, again assume the contrary that $\bar{b} \downarrow_{A \cup \cup_{m<n}} \bar{b}_{m} \bar{b}_{n}$ for some $n$. But now $\bar{b}_{n} \downarrow_{A} \bigcup_{m<n} \bar{b}_{m}$ by the definition of the sequence, and by symmetry and transitivity we get $\bar{b}_{n} \downarrow_{A} \bar{b} \cup \bigcup_{m<n} \bar{b}_{m}$. Then $\bar{b} \downarrow_{A} \bar{b}_{n}$ by monotonicity and symmetry, a contradiction. This proves the second claim. To prove the first claim we define increasing and finite sets $A_{n}:=$ $A \cup \bigcup_{m<n} \bar{b}_{m}$. Now $\bar{b} \downarrow_{A_{n}} A_{n+1}$ for each $n<\omega$, a contradiction with (1).

Item (5) follows from (2) by Corollary 3.4 and Theorem 3.20, where we need tameness. Item (2) follows from (5) by Proposition 3.26, since weak superstability clearly implies weak stability.

We note that in the previous theorem implication from (4) to (2) also holds without the Tarski-Vaught property.

3.4. a-categoricity. One of the basic results for abstract elementary classes with amalgamation, joint embedding and arbitrarily large models, shown by Shelah, is that categoricity in any uncountable cardinal implies stability in $\operatorname{LS}(\mathbb{K})$. We also proved in [11] that in our case stability in $\mathrm{LS}(\mathbb{K})=\aleph_{0}$ implies weak stability in each infinite cardinal. Since we now want to study the case without $\aleph_{0}$-stability, we will consider a weakening of categoricity called $a$-categoricity, and study when a-categoricity implies superstability. We recall that a model is said to be a-saturated if every Lascar strong type over a finite subset is realized in the model.

Definition 3.30. We say that the class $(\mathbb{K}, \preccurlyeq \mathbb{K})$ is a-categorical in $\kappa$ if there are exactly one a-saturated model of size $\kappa$, up to isomorphism.

Let us denote by $\mathrm{L}(\mathbb{K})$ the supremum of the number of Lascar strong types over any finite set. For any single finite set $E$, we know by Lemma 2.4 that the number of Lascar strong types over this set is strictly less than the number $\mathrm{H}=\beth_{\left(2^{\left.\aleph_{0}\right)^{+}}\right.}$. To count the value of $\mathrm{L}(\mathbb{K})$, we should take the supremum over each finite subset in the monster model $\mathfrak{M}$. On the other hand, if there is an automorphism $f \in \operatorname{Aut}(\mathfrak{M})$ mapping a finite set $E_{1}$ to another finite set $E_{2}$, there are exactly the same number of Lascar strong types over $E_{1}$ and $E_{2}$. Since there are at most $2^{\aleph_{0}}$ different isomorphism 
types of countable structures in $\mathbb{K}$, there are at most $\aleph_{0}$ times $2^{\aleph_{0}}$ finite sets in the monster model, up to automorphism. Now since $\operatorname{cf}(\mathrm{H})>2^{\aleph_{0}}$, we get

$$
\mathrm{L}(\mathbb{K})<\mathrm{H} .
$$

There exists an a-saturated model in every cardinal $\kappa \geq \mathrm{L}(\mathbb{K})$.

When $(\mathbb{K}, \preccurlyeq \mathbb{K})$ is a finitary abstract elementary class, we can study the class $\left(\mathbb{K}_{\mathrm{a}}, \preccurlyeq \mathbb{K}\right)$, where $\mathbb{K}_{\mathrm{a}}$ is the class of a-saturated models of $\mathbb{K}$. The class $\left(\mathbb{K}_{\mathrm{a}}, \preccurlyeq_{\mathbb{K}}\right)$ is an abstract elementary class with amalgamation, joint embedding, arbitrarily large models and $\operatorname{LS}\left(\mathbb{K}_{\mathrm{a}}\right)=\mathrm{L}(\mathbb{K})$. Also $\mathrm{LS}(\mathbb{K})$-tameness of $(\mathbb{K}, \preccurlyeq \mathbb{K})$ implies LS $\left(\mathbb{K}_{\mathrm{a}}\right)$-tameness for $\left(\mathbb{K}_{\mathrm{a}}, \preccurlyeq \mathbb{K}\right)$. Many results from more general theory of abstract elementary classes can be adapted for $\left(\mathbb{K}_{\mathrm{a}}, \preccurlyeq \mathbb{K}\right)$. Also a-categoricity transfer follows for certain cardinals (see Theorem 4.12).

We state the following results which adapt the presentation theorem and the construction of Ehrenfeucht-Mostowski models for AECs by Shelah.

Proposition 3.31. There is a class $\mathbb{K}^{*}$ of $\tau^{*}$-structures with $\tau^{*}=\tau \cup$ $\left\{F_{i}^{k}: k<\omega, i<\mathrm{L}(\mathbb{K})\right\}$, where each $F_{i}^{k}$ is a $k$-ary function symbol and the following holds.

(1) If $\mathscr{A}^{*} \in \mathbb{K}^{*}$ and $B \subset \mathscr{A}^{*}$ a subset such that $B$ is closed under functions $F_{i}^{k}$, then $B$ is an a-saturated $\mathbb{K}$-elementary substructure of $\mathscr{A}^{*} \uparrow \tau$.

(2) For every a-saturated $\mathscr{A} \in \mathbb{K}$ there is $\mathscr{A}^{*} \in \mathbb{K}^{*}$ such that $\mathscr{A}^{*} \uparrow \tau=\mathscr{A}$.

When $\mathscr{A}^{*} \in \mathbb{K}^{*}$ and $A \subset \mathscr{A}^{*}$, we denote by $S H(A)$ the closure of $A$ under the functions $F_{i}^{k}, k<\omega, i<\mathrm{L}(\mathbb{K})$. By the previous theorem, $S H(A)$ is an a-saturated $\mathbb{K}$-elementary substructure of $\mathscr{A}\lceil\tau$. The following formulation of the Ehrenfeucht-Mostowski model construction is tailored for the purposes of this paper. The proof of this theorem is similar to the proof of Proposition 2.13 in [12]. First we recall the concept of a tidy sequence from [12].

Definition 3.32. Let $i_{0}^{\alpha}<\cdots<i_{n}^{\alpha} \in I$ for each $\alpha<\lambda$, where $I$ is a linear order. We say that the sequence $\left(i_{0}^{\alpha}, \ldots, i_{n}^{\alpha}\right)_{\alpha<\lambda}$ is tidy if for each $0 \leq k \leq n$ one of the following holds.

(1) The index at $k$ is constant, that is, $i_{k}^{\alpha}=\beta \in I$ is fixed for each $\alpha<\lambda$.

(2) The index at $k$ is included in some $(m+1)$-block, that is,

$$
k \in\{p, p+1, \ldots, p+m\}
$$

where

(a) $p+m+1>n$ or for each $\beta<\lambda$, we have

$$
i_{p+m+1}^{\beta} \geq \sup \left\{i_{p+m}^{\alpha}: \alpha<\lambda\right\},
$$


(b) $p-1<0$ or for each $\beta<\lambda$, we have

$$
i_{p-1}^{\beta}<\min \left\{i_{p}^{\alpha}: \alpha<\lambda\right\},
$$

(c) for each $\alpha<\beta<\lambda$, we have $i_{p}^{\alpha}<\cdots<i_{p+m}^{\alpha}<i_{p}^{\beta}<\cdots<i_{p+m}^{\beta}$.

Proposition 3.33. For any linear order $I$ and set $A$ there is a sequence $\left(a_{i}\right)_{i \in I}$ and a model $\operatorname{EM}(I, A) \in \mathbb{K}^{*}$ with $A \cup\left(a_{i}\right)_{i \in I} \subset E M(I, A)$ such that

(1) $|\operatorname{EM}(I, A)|=|I|+|A|+\mathrm{L}(\mathbb{K})$.

(2) Each element in $\operatorname{EM}(I, A)$ is a $\tau^{*}$-term from some $a_{i_{0}}, \ldots, a_{i_{n}}$ and $\bar{a}$ with $n<\omega, i_{0}<\cdots<i_{n} \in I$ and $\bar{a} \in A$.

(3) Each partial order-preserving $f: I \rightarrow I$ extends to an $\tau^{*}$-isomorphism

$$
F: S H\left(\left\{a_{i}: i \in \operatorname{dom}(f)\right\} \cup A\right) \rightarrow S H\left(\left\{a_{i}: i \in \operatorname{rng}(f)\right\} \cup A\right)
$$

mapping $a_{i}$ to $a_{f(i)}$ for each $i \in \operatorname{dom}(f)$ and fixing $A$ pointwise.

(4) Let $\left(i_{0}^{\alpha}, \ldots, i_{n}^{\alpha}\right)_{\alpha<\lambda}, i_{0}^{\alpha}<\cdots<i_{n}^{\alpha} \in I$, be a tidy sequence and let

$$
\bar{b}_{i}=\bar{t}\left(a_{i_{0}^{\alpha}}, \ldots, a_{i_{n}^{\alpha}}\right)
$$

for a fixed sequence $\bar{t}$ of terms of $\tau^{*}$. Then $\left(\bar{b}_{i}\right)_{i<\alpha}$ is a strongly A-indiscernible sequence.

We identify $\left(a_{i}\right)_{i \in I}$ with $I$. The proof of the following theorem is standard, using Ehrenfeucht-Mostowski models.

TheOrEm 3.34 (Shelah). Let $(\mathbb{K} \preccurlyeq \mathbb{K})$ be an AEC with amalgamation, joint embedding, arbitrarily large models and $\mathrm{LS}(\mathbb{K}) \leq \mathrm{L}(\mathbb{K})$. Assume that the class $(\mathbb{K}, \preccurlyeq \mathbb{K})$ is a-categorical in some $\kappa>\mathrm{L}(\mathbb{K})$ and let $\kappa>\mu \geq \mathrm{L}(\mathbb{K})$. Then $\left(\mathbb{K}, \preccurlyeq_{\mathbb{K}}\right)$ is Galois-stable in $\mu$.

From this theorem it follows that when a simple finitary AEC $(\mathbb{K}, \preccurlyeq \mathbb{K})$ is a-categorical for some $\kappa>\mathrm{L}(\mathbb{K})$, it is weakly stable, and we can use the restricted properties of $\downarrow$ studied in Section 2.2. We also get the following corollary as usual.

COROLlary 3.35. Let $(\mathbb{K} \preccurlyeq \mathbb{K})$ be an AEC with amalgamation, joint embedding, arbitrarily large models and $\mathrm{LS}(\mathbb{K}) \leq \mathrm{L}(\mathbb{K})$. Assume that $\left(\mathbb{K}, \preccurlyeq_{\mathbb{K}}\right)$ is a-categorical in some $\kappa>\mathrm{L}(\mathbb{K})$ and let $\mu$ be such that $\mathrm{cf}(\kappa) \geq \mu$. Then the categorical a-saturated model of size $\kappa$ is $\mu$-saturated with respect to Galois types.

The next lemma gives another property that we want the a-categorical model to have.

Lemma 3.36. Assume that $\left(\mathbb{K}, \preccurlyeq_{\mathbb{K}}\right)$ is a simple finitary AEC. Let $\lambda>$ $\mathrm{L}(\mathbb{K})$. There is an a-saturated model $\mathscr{A}$ of size $\lambda$ with the following property: For any $A \subset \mathscr{A}$ such that $|A| \leq \mathrm{L}(\mathbb{K})$, any finite $B \subset A$ and any tuple $\bar{a}$ there is $\bar{b} \in \mathscr{A}$ realizing $\operatorname{Lstp}(\bar{a} / B)$ such that $\bar{b} \downarrow_{B} A$. 
Proof. We construct the model $\mathscr{A}$ as an increasing and continuous union of a-saturated models $\mathscr{A}_{i}$ of size $\lambda$, for $i<\mathrm{L}(\mathbb{K})^{+}$, such that the following holds: for any finite $B \subset \mathscr{A}_{i}$ and any tuple $\bar{a}$ there is $\bar{b} \in \mathscr{A}_{i+1}$ realizing $\operatorname{Lstp}(\bar{a} / B)$ such that $\bar{b} \downarrow_{B} \mathscr{A}_{i}$. Then since $\mathrm{L}(\mathbb{K})^{+}$is regular, for any $A \subset \mathscr{A}$ such that $|A| \leq \mathrm{L}(\mathbb{K})$ we can find $i<\mathrm{L}(\mathbb{K})^{+}$such that $A \subset \mathscr{A}_{i}$. We see that the model $\mathscr{A}$ is as required by monotonicity.

The construction is as follows. First let $\mathscr{A}_{0}$ be any a-saturated model of size $\lambda$. At the limit step we take union, so it is enough to construct the model at each successor step. Assume we have defined $\mathscr{A}_{i}$. Let $\left(B_{j}\right)_{j<\lambda}$ enumerate all finite subsets of $\mathscr{A}_{i}$. Then let $\left(\bar{a}_{k}^{j}\right)_{k<\mathrm{L}(\mathbb{K})}$ enumerate representatives for each Lascar strong type over a set $B_{j}$. For any finite $B_{i} \subset \mathscr{A}_{i}$ and a tuple $\bar{a}_{k}^{j}$, there is some $\bar{b}_{k}^{j}$ realizing $\operatorname{Lstp}\left(\bar{a}_{k}^{j} / B_{j}\right)$ such that $\bar{b}_{k}^{j} \downarrow_{B_{j}} \mathscr{A}_{i}$. This follows from simplicity and Corollary 2.12. Now let $\mathscr{A}_{i+1}$ be an a-saturated model of size $\lambda$ containing

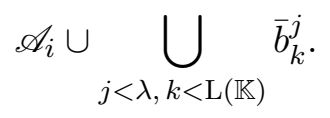

The construction is complete.

In the following proposition we assume that the a-categoricity cardinal has uncountable cofinality. This is needed to ensure that the categorical a-saturated model satisfies all weak types over countable subsets. Hence we are only able to prove superstability from a-categoricity in a cardinal with uncountable cofinality. This is a flaw also in our a-categoricity transfer theorem, and we would like to know whether it is possible to drop this assumption.

Proposition 3.37. Let $(\mathbb{K}, \preccurlyeq \mathbb{K})$ be a simple finitary AEC. Assume that $(\mathbb{K}, \preccurlyeq \mathbb{K})$ is a-categorical in $\kappa>\mathrm{L}(\mathbb{K})$ with uncountable cofinality. For each $\bar{a}$ and finite $A$ there is a strongly $A$-indiscernible sequence $\left(\bar{a}_{i}\right)_{i<\omega}$ such that $\bar{a}_{0}=\bar{a}$ and for any $\bar{b}$, the set $\left\{i<\omega: \bar{b} \downarrow_{A} \bar{a}_{i}\right\}$ is finite.

Proof. Let $I=\mathbb{Q}+\kappa+\omega$. The model $\operatorname{EM}(I, A)$ has size $\kappa$ and thus is the one a-saturated model of size $\kappa$. This model has the property of Lemma 3.36 and is $\aleph_{1}$-saturated by Corollary 3.35 . It is enough to study any $\bar{a}^{\prime}$ realizing $\operatorname{tp}^{\mathrm{w}}(\bar{a} / A)$, and hence, by Lemma 3.36 , we may assume that $\bar{a} \subset \operatorname{EM}(I, A)$ and $\bar{a} \downarrow_{A} S H(\mathbb{Q} \cup A)$.

Let $\bar{a}=\bar{t}\left(i_{0}, \ldots, i_{n}, A_{0}\right)$, where $\bar{t}$ is a sequence of terms of $\tau^{*}, i_{0}<$ $\cdots<i_{n} \in I$ and $A_{0} \subset A$. We can definite a tidy sequence $\left(j_{0}^{m}, \ldots, j_{n}^{m}\right)_{m<\omega}$, $j_{0}^{m}<\cdots<j_{n}^{m} \in I$, such that

(1) $j_{k}^{0}=i_{k}$ for each $0 \leq k \leq n$,

(2) $j_{k}^{m+1}=i_{k}$ is constant if and only if $i_{k} \in \mathbb{Q}$,

(3) when $k$ is minimal such that $i_{k} \notin \mathbb{Q}$, the indices at $k, \ldots, n$ form an $((n-k)+1)$-block which is cofinal in $\kappa+\omega$. 
Now the sequence $\left(\bar{a}_{m}\right)_{m<\omega}$, where $\bar{a}_{m}=\bar{t}\left(j_{0}^{m}, \ldots, j_{n}^{m}, A_{0}\right)$, is strongly $A$ indiscernible by Proposition 3.33(4). Also $\bar{a}_{0}=\bar{a}$. Since for any $m<\omega$ we have a $\tau^{*}$-isomorphism

$$
h: S H\left(\mathbb{Q} \cup A \cup\left\{j_{0}^{m}, \ldots, j_{n}^{m}\right\}\right) \rightarrow S H\left(\mathbb{Q} \cup A \cup\left\{j_{0}^{0}, \ldots, j_{n}^{0}\right\}\right)
$$

fixing $\mathbb{Q} \cup A$ and mapping $\bar{a}_{m}$ to $\bar{a}$, it follows that $\bar{a}_{m} \downarrow_{A} S H(\mathbb{Q} \cup A)$ for each $m<\omega$.

We claim that $\left(\bar{a}_{m}\right)_{m<\omega}$ is the sequence we need for the proof. To prove the claim, let $\bar{b}$ be any tuple. Again it is enough to study any $\bar{b}^{\prime}$ realizing $\operatorname{tp}^{\mathrm{w}}\left(\bar{b} / A \cup\left(\bar{a}_{m}\right)_{m<\omega}\right)$, and since $E M(I, A)$ is $\aleph_{1}$-saturated, we may assume that $\bar{b} \in E M(I, A)$. Now $\bar{b}=\bar{t}^{\prime}\left(h_{0}, \ldots, h_{p}, A^{\prime}\right)$ for some sequence $\bar{t}^{\prime}$ of terms of $\tau^{*}, h_{0}<\cdots<h_{p} \in I$ and $A^{\prime} \subset A$. We assume to the contrary that $\bar{b} \downarrow_{A} \bar{a}_{m}$ for infinitely many $m<\omega$. But then by (3) we can find $m<\omega$ such that $\bar{b}\rfloor_{A} \bar{a}_{m}$ and $h_{0}<\cdots<h_{p}<j_{k}^{m}$ for all $k$ such that $j_{k}^{m} \notin \mathbb{Q}$. There is a partial order-preserving $f: I \rightarrow I$ fixing $j_{0}^{m}, \ldots, j_{n}^{m}$ and mapping $h_{k}$ into $\mathbb{Q}$ for each $0 \leq k \leq p$. By Proposition 3.33(3), this extends to a $\tau^{*}$-isomorphism $F$ with domain $S H\left(\left\{j_{0}^{m}, \ldots, j_{n}^{m}, h_{0}, \ldots, h_{p}\right\} \cup A\right)$, fixing $\bar{a}_{m} \cup A$ and mapping $\bar{b}$ into $S H(\mathbb{Q} \cup A)$. Furthermore, since $\operatorname{dom}(F)$ and $\operatorname{rng}(F)$ are models, $F$ extends to an automorphism of $\mathfrak{M}$. By invariance we get $F(\bar{b}) \downarrow_{A} \bar{a}_{m}$. But on the other hand, since $F(\bar{b}) \in S H(\mathbb{Q} \cup A), \bar{a}_{m} \downarrow_{A} F(\bar{b})$. This is a contradiction with finite symmetry.

TheOREM 3.38. Assume that a simple finitary $(\mathbb{K}, \preccurlyeq \mathbb{K})$ is a-categorical in $\kappa \geq \mathrm{H}$ with uncountable cofinality. Then $(\mathbb{K}, \preccurlyeq \mathbb{K})$ is superstable.

Proof. Since $\operatorname{cf}(\mathrm{H})>\omega$ and $\max \left\{2^{\aleph_{0}}, \mathrm{~L}(\mathbb{K})\right\}<\mathrm{H}$, we have

$$
\left(\max \left\{2^{\aleph_{0}}, \mathrm{~L}(\mathbb{K})\right\}\right)^{+\omega}<\mathrm{H} \leq \kappa .
$$

Define $\lambda=\left(\max \left\{2^{\aleph_{0}}, \mathrm{~L}(\mathbb{K})\right\}\right)^{+\omega}$. By Theorem 3.34, ( $\left.\mathbb{K}, \preccurlyeq \mathbb{K}\right)$ is stable in $\lambda<\lambda^{\aleph_{0}}$. Now by Proposition 3.37, for any $\bar{a}$ and finite $A$ such that $\operatorname{tp}^{\mathrm{w}}(\bar{a} / A)$ is unbounded, there is a strongly $A$-indiscernible sequence $\left(\bar{a}_{i}\right)_{i<\omega}$ such that for any $\bar{b}$, the set $\left.\{i<\omega: \bar{b}\rfloor_{A} \bar{a}_{i}\right\}$ is finite. Also $\aleph_{0}^{\aleph_{0}} \leq \lambda$. Then by Lemma $3.7,(\mathbb{K}, \preccurlyeq \mathbb{K})$ is superstable.

In the above theorem it is enough that $(\mathbb{K}, \preccurlyeq \mathbb{K})$ is a-categorical in some $\kappa>\lambda \geq \max \left\{\mathrm{L}(\mathbb{K}), 2^{\aleph_{0}}\right\}$, where $\lambda^{\aleph_{0}}>\lambda$ and $\operatorname{cf}(\kappa)>\omega$.

We will prove an a-categoricity transfer result in Section 4.1. For this we need that under superstability, the a-categorical model of size $>\mathrm{L}(\mathbb{K})$ is $\mathrm{L}(\mathbb{K})^{+}$-saturated with respect to weak Lascar strong types. We will prove a stronger result: the a-categorical model is strongly saturated. We say that $\mathscr{A}$ is strongly saturated if all weak Lascar strong types over subsets of size $<|\mathscr{A}|$ are realized in $\mathscr{A}$. We also say that $(\mathbb{K}, \preccurlyeq \mathbb{K})$ is strongly stable in $\lambda$ if there are at most $\lambda$-many weak Lascar strong types over a model of size $\lambda$. 
TheOREM 3.39. Assume that $(\mathbb{K}, \preccurlyeq \mathbb{K})$ is a superstable simple finitary $A E C$ and $\lambda>\mathrm{L}(\mathbb{K})$. There is a strongly saturated model of size $\lambda$.

Proof. In Corollary 3.4 we show that $\left(\mathbb{K}, \preccurlyeq_{\mathbb{K}}\right)$ is strongly stable in each cardinal $\lambda \geq \mathrm{L}(\mathbb{K})$. Let $\lambda>\mathrm{L}(\mathbb{K})$ and let $\left(\mathscr{A}_{i}\right)_{i<\lambda}$ be an increasing and continuous chain of models of size $\lambda$ such that each weak Lascar strong type over $\mathscr{A}_{i}$ is realized in $\mathscr{A}_{i+1}$. We claim that $\mathscr{A}=\bigcup_{i<\lambda} \mathscr{A}_{i}$ is strongly saturated. If $\lambda$ is regular, this is clear. We may assume that $\lambda$ is a limit.

Let $\bar{a}$ be a tuple and $B \subset \mathscr{A}$ such that $|B|<\lambda$. We want to realize $\operatorname{Lstp}^{\mathrm{w}}(\bar{a} / B)$ in $\mathscr{A}$. By local character for models and since $\lambda$ is a limit ordinal, there is $\gamma<\lambda$ such that $\bar{a} \downarrow_{\mathscr{A}_{\gamma}} \mathscr{A}$. Set $\alpha=\gamma+|B|^{+}<\lambda$. Similarly for any finite $\bar{c}$, there is $i<\alpha$ such that $\bar{c} \downarrow_{\mathscr{A}_{i}} \mathscr{A}_{\alpha}$. Since $\operatorname{cf}(\alpha)>|B|$, there is $\beta$ such that $\gamma \leq \beta<\alpha$ and

$$
\bar{c} \downarrow_{\mathscr{A}_{\beta}} \mathscr{A}_{\alpha} \quad \text { for each finite tuple } \bar{c} \in B .
$$

Choose $\bar{b} \in \mathscr{A}_{\beta+1}$ realizing $\operatorname{Lstp}^{\mathrm{w}}\left(\bar{a} / \mathscr{A}_{\beta}\right)$. Then $\bar{c} \downarrow_{\mathscr{A}_{\beta}} \bar{b}$ for each finite tuple $\bar{c} \in B$. By symmetry and finite character over models, $\bar{b} \downarrow_{\mathscr{A}_{\beta}} B$. Furthermore by stationarity, $\bar{b}$ is the realization of $\operatorname{Lstp}^{\mathrm{w}}(\bar{a} / B)$ in $\mathscr{A}$.

To justify the notion of a-categoricity, we give an example of a $L_{\omega_{1} \omega^{-}}$ definable class of structures which is not categorical but is a-categorical in each cardinal $>2^{\aleph_{0}}$.

EXAMPLE 3.40. Let $F$ and $E_{i}, i \leq \omega$, be binary relation symbols. Let $T$ be the following set of axioms:

(1) Axioms stating that each $E_{i}$ and $F$ are equivalence relations.

(2) $E_{0}$ divides the structure into two classes, that is,

$$
\exists x \exists y\left(\neg E_{0}(x, y) \wedge \forall z\left(E_{0}(x, z) \vee E_{0}(y, z)\right)\right) .
$$

(3) The relation $E_{n+1}$ divides all classes of $E_{n}$ into two, that is, for all $n<\omega$,

$$
\begin{array}{r}
\forall x \exists y \exists z\left(E_{n}(y, x) \wedge E_{n}(z, x) \wedge \neg E_{n+1}(y, z) \wedge\right. \\
\left.\forall x^{\prime}\left(E_{n}\left(x^{\prime}, x\right) \leftrightarrow\left(E_{n+1}\left(x^{\prime}, y\right) \vee E_{n+1}\left(x^{\prime}, z\right)\right)\right)\right) .
\end{array}
$$

(4) The relation $E_{\omega}$ is an intersection of the relations $E_{n}, n<\omega$, that is,

$$
\forall x \forall y\left(E_{\omega}(x, y) \leftrightarrow \bigwedge_{n<\omega} E_{n}(x, y)\right) .
$$

(5) All equivalence classes of $E_{\omega}$ are of equal size ( $F$ defines a one-to-one and onto function between any two classes), that is,

$$
\forall x \forall y \exists ! z\left(F(x, z) \wedge E_{\omega}(y, z)\right) .
$$

This example is not categorical, since in a model of $T$ it might happen that some intersection of equivalence classes corresponding to a branch in 
$2^{\omega}$ is empty. Not even the class of $\aleph_{0}$-saturated structures of this theory is categorical. If each Lascar strong type over the empty set is realized in a model of $T$, no empty intersections can occur. When $\mathscr{A}$ is an a-saturated model of $T$ and is of size $\kappa>2^{\aleph_{0}}$, then each equivalence class of $E_{\omega}$ must be of size $\kappa$. Thus all such models is isomorphic.

\section{PRIMARY MODELS}

In this section we assume that $(\mathbb{K}, \preccurlyeq \mathbb{K})$ is a simple, superstable, finitary AEC with the Tarski-Vaught property.

Definition 4.1. Let $\bar{a}$ be a tuple and $A$ a set. A weak Lascar strong type $\operatorname{Lstp}^{\mathrm{w}}(\bar{a} / A)$ is a-isolated over a finite $E \subset A$ if whenever $\bar{b}$ realizes $\operatorname{Lstp}(\bar{a} / E)$, then $\bar{b} \downarrow_{E} A$.

The property of being a-isolated is invariant under automorphisms, that is, if $\operatorname{Lstp}^{\mathrm{w}}(\bar{a} / A)$ is a-isolated over $E \subset A$ and $f \in \operatorname{Aut}(\mathfrak{M})$, then the type $\operatorname{Lstp}^{\mathrm{w}}(f(\bar{a}) / f(A))$ is a-isolated over $f(E)$.

Lemma 4.2. For every tuple $\bar{a}$, set $A$ and finite $B \subset A$ there is $\bar{b}$ and finite $A_{0} \subset A$ such that $\operatorname{Lstp}(\bar{b} / B)=\operatorname{Lstp}(\bar{a} / B)$ and $\operatorname{Lstp}^{\mathrm{w}}(\bar{b} / A)$ is aisolated over $A_{0}$.

Proof. Assume that $\bar{a}, A$ and finite $B \subset A$ witness the contrary. We define tuples $\bar{a}_{i}$ and finite sets $A_{i}$ for $i<\omega$ to contradict Proposition 3.11. First let $\bar{a}_{0}=\bar{a}$ and $A_{0}=B$. Then assume we have defined $\bar{a}_{n}$ and $A_{n}$ for $i \leq n$ such that

(1) $\operatorname{Lstp}\left(\bar{a}_{i} / B\right)=\operatorname{Lstp}(\bar{a} / B)$,

(2) the sets $A_{i}$ are finite and $A_{i} \subset A_{i+1} \subset A$,

(3) $\operatorname{Lstp}\left(\bar{a}_{i+1} / A_{i}\right)=\operatorname{Lstp}\left(\bar{a}_{i} / A_{i}\right)$,

(4) $\bar{a}_{i+1} \downarrow_{A_{i}} A_{i+1}$.

Since we have (1), the type $\operatorname{Lstp}^{\mathrm{w}}\left(\bar{a}_{n} / A\right)$ cannot be a-isolated over finite $A_{n} \subset A$. Thus there is a tuple $\bar{a}_{n+1} \operatorname{such}$ that $\operatorname{Lstp}\left(\bar{a}_{n+1} / A_{n}\right)=\operatorname{Lstp}\left(\bar{a}_{n} / A_{n}\right)$ but $\bar{a}_{n+1} \nmid A_{n} A$. Furthermore, by finite character of independence, there is a finite $A_{n+1} \subset A$ such that $\bar{a}_{n+1} \downarrow_{A_{n}} A_{n+1}$. We may assume that $A_{n} \subset A_{n+1}$. This construction contradicts Proposition 3.11.

Definition 4.3. We say that $\mathscr{A}$ is $\mathbf{S}$-primary over a set $A$ if for some ordinal $\xi$ there are tuples $\bar{a}_{i}$ and finite sets $A_{i}$ for $i<\xi$ such that

(1) the weak Lascar strong type $\operatorname{Lstp}^{\mathrm{w}}\left(\bar{a}_{i} / A \cup \bigcup_{j<i} \bar{a}_{j}\right)$ is a-isolated over $A_{i} \subset A \cup \bigcup_{j<i} \bar{a}_{j}$

(2) $\mathscr{A}=A \cup \bigcup_{i<\xi} \bar{a}_{i}$ is $\mathbf{S}$-saturated.

If in addition $\mathscr{A}$ is a-saturated, we say that it is a-primary. 
We say that $\mathscr{A}$ is a-constructible over $A$ if (1) in the above definition holds. Analogously to the similar result in [12], we can prove the following lemma.

Lemma 4.4. For every set $A$ there is a model $\mathscr{B}$ of size $|A|+\aleph_{0}$ which is S-primary over $A$. Furthermore, if $\mathscr{B}^{\prime}$ is an a-saturated model containing $A$, we can choose $\mathscr{B}$ such that $\mathscr{B} \preccurlyeq \mathbb{K} \mathscr{B}^{\prime}$.

Proof. We prove the last claim. Write $|A|+\aleph_{0}=\lambda$. By induction on $n<\omega$ we define sets $B_{n} \subset \mathscr{B}^{\prime}$ of size $\lambda$, tuples $\bar{a}_{i}^{n} \in \mathscr{B}^{\prime}$ and finite sets $A_{i}^{n} \subset \mathscr{B}^{\prime}$ for $i<\lambda$. First let $B_{0}=A$.

Assume we have defined $B_{n}$. Enumerate all finite subsets of $B_{n}$ as $\left(\bar{b}_{j}\right)_{j<\lambda}$ and let $\mathbf{S}=\left\{\phi_{k}: k<\omega\right\}$. Let $\left(\bar{c}_{j}^{k}\right)_{j<\lambda, k<\omega}$ be tuples such that whenever there exists a tuple $\bar{c}$ such that $\mathfrak{M} \models \phi_{k}\left(\bar{b}_{j}, \bar{c}\right)$ for $\phi_{k} \in \mathbf{S}$ and finite $\bar{b}_{j} \in B_{n}$, then one such $\bar{c}$ is listed as $\bar{c}_{j}^{k}$. If such a $\bar{c}$ does not exist, $\bar{c}_{j}^{k}$ can be arbitrary. Then let $\left(\bar{c}_{i}\right)_{i<\lambda}$ enumerate all $\left(\bar{c}_{j}^{i}\right)_{i<\omega, j<\lambda}$.

Let $\alpha<\lambda$ and assume we have defined $\bar{a}_{i}^{n}$ for $i<\alpha$. Let $\bar{c}_{j}^{i}$ be the tuple listed as $\bar{c}_{\alpha}$. We use Lemma 4.2 to find a tuple $\bar{d}$ realizing $\operatorname{Lstp}\left(\bar{c}_{j}^{i} / \bar{b}_{j}\right)$ and a finite subset $A_{\alpha}^{n} \subset B_{n} \cup \bigcup_{i<\alpha} \bar{a}_{i}^{n}$ such that $\operatorname{Lstp}^{\mathrm{w}}\left(\bar{d} / B_{n} \cup \bigcup_{i<\alpha} \bar{a}_{i}^{n}\right)$ is aisolated over $A_{\alpha}^{n}$. By a-saturation, there is $\bar{a}_{\alpha}^{n} \in \mathscr{B}^{\prime}$ realizing $\operatorname{Lstp}\left(\bar{d} / \bar{b}_{j} \cup A_{\alpha}^{n}\right)$. Then also $\operatorname{Lstp}^{\mathrm{w}}\left(\bar{a}_{\alpha}^{n} / B_{n} \cup \bigcup_{i<\alpha} \bar{a}_{i}^{n}\right)$ is a-isolated over $A_{\alpha}^{n}$. Finally, let $B_{n+1}=$ $B_{n} \cup \bigcup_{i<\lambda} \bar{a}_{i}^{n}$.

Clearly $\mathscr{B}=\bigcup_{n<\omega} B_{n}=A \cup \bigcup_{(n, i) \in \omega \times \lambda} \bar{a}_{i}^{n}$ is $\mathbf{S}$-saturated and thus a model. Now $\mathscr{B}$ is an S-primary model over $A$ and is of size $\lambda$.

We can easily see how to change the above construction to obtain an a-primary model. In $B_{n+1}$ we should also realize all Lascar strong types over finite subsets of $B_{n}$. We obtain the following result.

Lemma 4.5. For every set $A$ there is a model $\mathscr{B}$ of size $|A|+\mathrm{L}(\mathbb{K})$ which is a-primary over $A$. Furthermore, if $\mathscr{B}^{\prime}$ is an a-saturated model containing $A$, we can choose $\mathscr{B}$ such that $\mathscr{B} \preccurlyeq \mathbb{K} \mathscr{B}^{\prime}$.

We define domination as usual.

Definition 4.6. We say that a set $A$ dominates a set $B$ over an asaturated model $\mathscr{A}$ if for every tuple $\bar{c}$,

$$
\bar{c} \downarrow_{\mathscr{A}} A \Rightarrow \bar{c} \downarrow_{\mathscr{A}} B \text {. }
$$

We show that a-primary models have similar properties to f-primary models in [12]. Since the concept itself is here different, we need to reprove some of these.

Lemma 4.7. Let $B$ be a set and $A_{1}, A_{2} \subset B$ finite such that

(1) $\operatorname{Lstp}^{\mathrm{w}}\left(\bar{a}_{0} / B\right)$ is a-isolated over $A_{0}$,

(2) $\operatorname{Lstp}^{\mathrm{w}}\left(\bar{a}_{1} / B \cup \bar{a}_{0}\right)$ a-isolated over $A_{1} \cup \bar{a}_{0}$.

Then the type $\operatorname{Lstp}^{\mathrm{w}}\left(\bar{a}_{0}, \bar{a}_{1} / B\right)$ is a-isolated over $A=A_{1} \cup A_{2}$. 
Proof. We assume to the contrary that there is some finite tuple $\bar{c}_{0} \bar{c}_{1}$ realizing $\operatorname{Lstp}\left(\bar{a}_{0}, \bar{a}_{1} / A\right)$ such that $\bar{c}_{0} \bar{c}_{1} \downarrow_{A} B$. By finite character there is some $\bar{b} \in B$ such that

$$
\bar{c}_{0} \bar{c}_{1} \downarrow_{A} \bar{b} .
$$

There is $f \in \operatorname{Saut}(\mathfrak{M} / A)$ such that $f\left(\bar{c}_{0} \bar{c}_{1}\right)=\bar{a}_{0} \bar{a}_{1}$. By $1, \bar{c}_{0} \downarrow_{A} \bar{b}$ and then by invariance, $\bar{a}_{0} \downarrow_{A} f(\bar{b})$. Since also $\bar{a}_{0} \downarrow_{A} \bar{b}$, from symmetry and stationarity we get

$$
\operatorname{Lstp}\left(\bar{b} / A \cup \bar{a}_{0}\right)=\operatorname{Lstp}\left(f(\bar{b}) / A \cup \bar{a}_{0}\right) .
$$

Let $g$ be a strong automorphism mapping $f(\bar{b})$ to $\bar{b}$ and fixing $A \cup \bar{a}_{0}$. Now $\operatorname{Lstp}\left(g\left(\bar{a}_{1}\right) / A \cup \bar{a}_{0}\right)=\operatorname{Lstp}\left(\bar{a}_{1} / A \cup \bar{a}_{0}\right)$ and since by (2),

$$
g\left(\bar{a}_{1}\right) \downarrow_{A \cup \bar{a}_{0}} \bar{b},
$$

the pairs lemma implies that $g\left(\bar{a}_{1}\right) \bar{a}_{0} \downarrow_{A} \bar{b}$. Since $g^{-1} \in \operatorname{Aut}\left(\mathfrak{M} / \bar{a}_{0} \cup A\right)$ maps $\bar{b}$ to $f(\bar{b})$, we have $\bar{a}_{1} \bar{a}_{0} \downarrow_{A} f(\bar{b})$ by invariance. Again using the automorphism $f^{-1}$ and invariance, we infer that $\bar{c}_{0} \bar{c}_{1} \downarrow_{A} \bar{b}$, a contradiction.

Proposition 4.8. Let $\mathscr{A}$ be an a-saturated model and $B$ a set. Let

$$
\mathscr{A}^{*}=\mathscr{A} \cup B \cup \bigcup_{i<\xi} \bar{a}_{i}
$$

be a-constructible over $\mathscr{A} \cup B$ and let $\bar{d}$ be a tuple in $\mathscr{A}^{*}$. Then there are $\bar{a}=\bar{a}_{i_{0}}, \ldots, \bar{a}_{i_{n}}$ for $i_{0}<\cdots<i_{n}<\xi$, finite $A \subset \mathscr{A}$ and $\bar{b} \subset B$ such that

(1) $\bar{d} \subset A \cup \bar{b} \cup \bar{a}$

(2) $\operatorname{Lstp}^{\mathrm{w}}(\bar{a} / \mathscr{A} \cup \bar{b})$ is a-isolated over $A \cup \bar{b}$,

(3) the tuple $\bar{b}$ dominates $\bar{a} \cup \bar{b}$ over $\mathscr{A}$.

Proof. The proof of items (1) and (2) is identical to the proof of the analogous result in [12], using Lemma 4.7. We now assume that we have found $\bar{a}, \bar{b}$ and $A$ satisfying (1) and (2) and then show that (3) holds.

Assume to the contrary that $\bar{c} \downarrow_{\mathscr{A}} \bar{b}$ but $\bar{c} \downarrow \mathscr{A} \bar{b} \cup \bar{a}$ for some tuple $\bar{c}$. By symmetry, also $\bar{b} \downarrow_{\mathscr{A}} \bar{c}$. Let $A^{\prime} \subset \mathscr{A}$ be finite such that $A \subset A^{\prime}$, $\bar{c} \downarrow_{A^{\prime}} \mathscr{A} \cup \bar{b}$ and $\bar{b} \downarrow_{A^{\prime}} \mathscr{A} \cup \bar{c}$. By finite character, there is a finite $B^{\prime}$ such that $A^{\prime} \subset B^{\prime} \subset \mathscr{A}$ and $\bar{c} \downarrow_{A^{\prime}} B^{\prime} \cup \bar{a} \cup \bar{c}$.

Since $\mathscr{A}$ is a-saturated, there is $\bar{d} \in \mathscr{A}$ realizing $\operatorname{Lstp}\left(\bar{c} / B^{\prime}\right)$. Since $\bar{b} \downarrow_{B^{\prime}}$ $\mathscr{A} \cup \bar{c}$, we get $\bar{c} \downarrow_{B^{\prime}} \bar{b}$ and $\bar{d} \downarrow_{B^{\prime}} \bar{b}$ by symmetry. By stationarity there is an automorphism $g \in \operatorname{Aut}\left(\mathfrak{M} / B^{\prime} \cup \bar{b}\right)$ mapping $\bar{c}$ to $\bar{d}$.

By a-isolation, $g(\bar{a}) \downarrow_{A^{\prime} \cup \bar{b}} \mathscr{A}$, and furthermore $g(\bar{a}) \downarrow_{A^{\prime} \cup \bar{b}} \bar{d} \cup B^{\prime}$. On the other hand, by monotonicity and invariance, $\bar{d} \downarrow_{A^{\prime}} B^{\prime} \cup \bar{b}$, and by symmetry, $\bar{b} \cup B^{\prime} \downarrow_{A^{\prime}} \bar{d}$. Now by the pairs lemma, $g(\bar{a}) \cup \bar{b} \cup B^{\prime} \downarrow_{A^{\prime}} \bar{d}$.

But since $\bar{c} \downarrow_{A^{\prime}} \bar{a} \cup \bar{b} \cup B^{\prime}$, by invariance $\bar{d} \downarrow_{A^{\prime}} g(\bar{a}) \cup \bar{b} \cup B^{\prime}$, and by symmetry, $g(\bar{a}) \cup \bar{b} \cup B^{\prime} \downarrow_{A^{\prime}} \bar{d}$, a contradiction.

By finite character we obtain the following corollary. 
Corollary 4.9. Let $\mathscr{B}=\mathscr{A} \cup B \cup\left(\bar{a}_{i}\right)_{i<\xi}$ be an a-constructible model over $\mathscr{A} \cup B$, where $\mathscr{A}$ is an a-saturated model. Then $B$ dominates $\mathscr{B}$ over the model $\mathscr{A}$.

4.1. Morley sequences. In this section we assume that $(\mathbb{K}, \preccurlyeq \mathbb{K})$ is a simple, superstable finitary AEC. The following result is again adapted from Shelah for this context. We sketch the proof.

Proposition 4.10. Let $\lambda$ be a cardinal, $\mathscr{A}$ an a-saturated model, $|\mathscr{A}| \geq$ $\lambda^{+}>\mathrm{L}(\mathbb{K})$ and let $B \subset \mathscr{A}$ be such that $|B|<\lambda^{+}$. Then there is an asaturated model $\mathscr{B} \preccurlyeq \mathbb{K} \mathscr{A}$, finite $E \subset \mathscr{B}$ and a sequence $\left(\bar{a}_{i}\right)_{i<\lambda^{+}}$such that

$$
\operatorname{Lstp}^{\mathrm{w}}\left(\bar{a}_{i} / \mathscr{B}\right)=\operatorname{Lstp}^{\mathrm{w}}\left(\bar{a}_{0} / \mathscr{B}\right)
$$

and

$$
\bar{a}_{i} \downarrow_{E} \mathscr{B} \cup \bigcup_{j<i} \bar{a}_{j} \quad \text { for each } i<\lambda^{+} .
$$

Proof. Define a continuous and increasing chain of a-saturated models $\mathscr{A}_{i} \preccurlyeq \mathbb{K} \mathscr{A}$ and tuples $\bar{a}_{i} \in \mathscr{A}, i<\lambda^{+}$, such that $B \subset \mathscr{A}_{0},\left|\mathscr{A}_{i}\right|=|B|+\mathrm{L}(\mathbb{K})$ and $\bar{a}_{i} \in \mathscr{A}_{i+1} \backslash \mathscr{A}_{i}$. By superstability, for each $i$ there is a finite $E_{i} \subset \mathscr{A}_{i}$ such that $\bar{a}_{i} \downarrow_{E_{i}} \mathscr{A}_{i}$. By Fodor's lemma we may assume that $\lambda^{+}$-many $E_{i}$ are included in $\mathscr{A}_{i_{0}}$ for a fixed $i_{0}$. Taking a subsequence, we may assume that $i_{0}=0$ and furthermore, using the pigeon-hole principle, we may assume that $\bar{a}_{i} \downarrow_{E} \mathscr{A}_{i}$ for a fixed finite $E \subset \mathscr{A}_{0}$ and for each $i<\lambda^{+}$. Also since $\lambda^{+}>\mathrm{L}(\mathbb{K})$, we may assume that $\operatorname{Lstp}\left(\bar{a}_{i} / E\right)=\operatorname{Lstp}\left(\bar{a}_{j} / E\right)$ for each $i<j<\lambda^{+}$. Then by stationarity, $\operatorname{Lstp}^{\mathrm{w}}\left(\bar{a}_{i} / \mathscr{A}_{i}\right)=\operatorname{Lstp}^{\mathrm{w}}\left(\bar{a}_{j} / \mathscr{A}_{i}\right)$ for each $i<j<\lambda^{+}$. We can take $\mathscr{B}=\mathscr{A}_{0}$.

We call the sequence $(\bar{a})_{i<\lambda^{+}}$from the above proposition a Morley sequence over $\mathscr{B}$. The finite set $E \subset \mathscr{B}$ is called the base set.

LEMma 4.11. Let $\left(\bar{a}_{i}\right)_{i<\alpha}$ be a Morley sequence over an a-saturated $\mathscr{B}$, and let $E \subset \mathscr{B}$ be the base set. Then for all $n<\omega$ and $j_{0}<\cdots<j_{n}<\alpha$,

(1) $\bar{b}_{j_{0}}, \ldots, \bar{b}_{j_{n}} \downarrow_{E} \mathscr{B}$

(2) $\operatorname{Lstp}^{\mathrm{w}}\left(\bar{b}_{j_{0}}, \ldots, \bar{b}_{j_{n}} / \mathscr{B}\right)=\operatorname{Lstp}^{\mathrm{w}}\left(\bar{b}_{0}, \ldots, \bar{b}_{n} / \mathscr{B}\right)$.

Proof. Item (1) can be shown by induction on $n$, using the pairs lemma. We also prove (2) by induction on $n$. The case $n=0$ is clear by definition. We assume that (2) holds for $n$. To prove it for $n+1$, let $j_{0}<\cdots<j_{n}<$ $j_{n+1}<\alpha$. Let $C \subset \mathscr{B}$ be an arbitrary finite subset. By induction, there is $f \in \operatorname{Saut}(\mathfrak{M} / C \cup E)$ such that $f\left(\bar{b}_{j_{k}}\right)=\bar{b}_{k}$ for $0 \leq k \leq n$. By invariance,

$$
f\left(\bar{b}_{j_{n+1}}\right) \downarrow_{E} C \cup \bar{b}_{0}, \ldots, \bar{b}_{n},
$$

and then by stationarity,

$$
\operatorname{Lstp}\left(f\left(\bar{b}_{j_{n+1}}\right) / E \cup C \cup \bar{b}_{n}, \ldots, \bar{b}_{0}\right)=\operatorname{Lstp}\left(\bar{b}_{n+1} / E \cup C \cup \bar{b}_{n}, \ldots, \bar{b}_{0}\right) .
$$


Hence $\operatorname{Lstp}\left(\bar{b}_{j_{n+1}}, \ldots, \bar{b}_{j_{0}} / C\right)=\operatorname{Lstp}\left(f\left(\bar{b}_{j_{n+1}}\right), \bar{b}_{n}, \ldots, \bar{b}_{0} / C\right)$, and also

$$
\operatorname{Lstp}\left(\bar{b}_{j_{n+1}}, \ldots, \bar{b}_{j_{0}} / C\right)=\operatorname{Lstp}\left(\bar{b}_{n+1}, \bar{b}_{n}, \ldots, \bar{b}_{0} / C\right) .
$$

4.2. a-categoricity transfer. We use a-primary models to prove an a-categoricity transfer theorem for simple tame finitary AECs. Grossberg and VanDieren have already a categoricity transfer result in [7] for tame classes which can be applied here. The class of a-saturated models of a simple tame finitary AECs with the induced notion $\preccurlyeq_{\mathbb{K}}$ forms an abstract elementary class with amalgamation, joint embedding, arbitrarily large models, Löwenheim-Skolem number $\mathrm{L}(\mathbb{K})$ and tameness in $\mathrm{L}(\mathbb{K})$. Thus the result of [7] implies that a-categoricity in a successor cardinal strictly greater than $\mathrm{L}(\mathbb{K})^{+}$gives upwards a-categoricity transfer. Furthermore, we then get acategoricity for all cardinals above $\mathrm{H}_{2}(\mathrm{~L}(\mathbb{K}))$ by the downward categoricity transfer result presented by Shelah [22] (see also Baldwin [2]). Combining these results we get the following theorem.

Theorem 4.12 (Grossberg, Baldwin, Shelah, VanDieren). Let ( $\mathbb{K}, \preccurlyeq \mathbb{K}$ ) be an AEC with amalgamation, joint embedding, arbitrarily large models and tameness in $\chi$. Assume that $(\mathbb{K}, \preccurlyeq \mathbb{K})$ is a-categorical in a successor cardinal $\kappa^{+}>\max \left\{\mathrm{L}(\mathbb{K})^{+}, \chi\right\}$. Then it is a-categorical in every

$$
\lambda \geq \min \left\{\kappa^{+}, \mathrm{H}_{2}(\mathrm{~L}(\mathbb{K}))\right\} .
$$

Here $\mathrm{H}_{2}(\mathrm{~L}(\mathbb{K}))$ is the second Hanf number for the class $\left(\mathbb{K}_{\mathrm{a}}, \preccurlyeq \mathbb{K}\right)$, that is, $\mathrm{H}(\mathrm{H}(\mathrm{L}(\mathbb{K})))$. Our result does not assume the a-categoricity cardinal being a successor, but we still have to make some assumptions on the cardinal. Also the class studied in [7] is more general.

The following proposition is an analogue of the weak categoricity transfer of [12]. Tameness is not needed for this proposition.

Proposition 4.13. Let $(\mathbb{K}, \preccurlyeq \mathbb{K})$ be a simple, superstable finitary $A E C$ with the Tarski-Vaught property. Assume that there is $\kappa>\mathrm{L}(\mathbb{K})$ such that each a-saturated model of size $\kappa$ realizes all weak Lascar strong types over subsets of size $\leq \mathrm{L}(\mathbb{K})$. Then any a-saturated model $\mathscr{A}$ such that $|\mathscr{A}|>\mathrm{L}(\mathbb{K})$ is saturated with respect to weak Lascar strong types.

Proof. Let $\mathscr{A}$ be an a-saturated model such that $|\mathscr{A}|>\mathrm{L}(\mathbb{K})$ and let $B \subset \mathscr{A},|B|<|\mathscr{A}|$. Let also $\bar{d} \in \mathfrak{M}$ be a finite tuple. By Proposition 4.10 there is a Morley sequence $\left(\bar{b}_{i}\right)_{i<\omega} \subset \mathscr{A}$ over an a-saturated model $\mathscr{B} \preccurlyeq \mathbb{K} \mathscr{A}$ containing $B$. Let $E_{1}$ be the base set. By local character, there is a finite $E_{2}$ such that $\bar{d} \downarrow_{E_{2}} \mathscr{B}$. We show that $\operatorname{Lstp}^{\mathrm{w}}(\bar{d} / \mathscr{B})$ is realized in $\mathscr{A}$.

Let $\mathscr{C} \preccurlyeq \mathbb{K} \mathscr{B}$ be a-saturated and of size $\mathrm{L}(\mathbb{K})$ such that $E_{1} \cup E_{2} \subset \mathscr{C}$. We use extension to continue the Morley sequence to $\left(\bar{b}_{i}\right)_{i<\kappa}$. Let

$$
\mathscr{C}^{*}=\mathscr{C} \cup \bigcup_{i<\kappa} \bar{b}_{i} \cup \bigcup_{j<\xi} \bar{a}_{j}
$$


be a-primary over $\mathscr{C} \cup \bigcup_{i<\kappa} \bar{b}_{i}$ such that $\left|\mathscr{C}^{*}\right|=\kappa$. By assumption, the model $\mathscr{C}^{*}$ is $\mathrm{L}(\mathbb{K})^{+}$-saturated with respect to weak Lascar strong types. Let $\bar{d}^{*} \in \mathscr{C}^{*}$ realize $\operatorname{Lstp}^{\mathrm{w}}(\bar{d} / \mathscr{C})$. We find finite $A \subset \mathscr{C}, \bar{b}=\left(b_{i_{0}}, \ldots, b_{i_{m}}\right)$, $i_{0}<\cdots<i_{m}<\kappa$, and $\bar{a}=\left(\bar{a}_{j_{0}}, \ldots, \bar{a}_{j_{n}}\right), j_{0}<\cdots<j_{n}<\xi$, as in Proposition 4.8. We use again local character to find a finite $E_{3} \subset \mathscr{C}$ such that $\bar{a}^{\frown} \bar{b} \downarrow_{E_{3}} \mathscr{C}$.

Define $\bar{b}^{*}=\left(b_{0}, \ldots, b_{m}\right) \in \mathscr{A}$. By Lemma 4.11(2), we have

$$
\operatorname{Lstp}^{\mathrm{w}}(\bar{b} / \mathscr{C})=\operatorname{Lstp}^{\mathrm{w}}\left(\bar{b}^{*} / \mathscr{C}\right) \text {, }
$$

and thus there is $f \in \operatorname{Saut}\left(\mathfrak{M} / E_{1} \cup E_{3}\right)$ such that $f(\bar{b})=\bar{b}^{*}$. By Corollary 2.12 and simplicity, there is $\bar{a}^{\prime}$ realizing $\operatorname{Lstp}\left(f(\bar{a}) / E_{1} \cup E_{3} \cup \bar{b}^{*}\right)$ such that $\bar{a}^{\prime} \downarrow_{E_{1} \cup E_{3} \cup \bar{b}^{*}} \mathscr{C}$. By Lemma 4.11(1), $\bar{b}^{*} \downarrow_{E_{1}} \mathscr{C}$, and thus by the pairs lemma, $\bar{a}^{\prime} \frown \bar{b}^{*} \downarrow_{E_{1} \cup E_{3}} \mathscr{C}$. But now by stationarity,

$$
\left.\operatorname{Lstp}^{\mathrm{w}}\left(\bar{a}^{\prime} \frown \bar{b}^{*} / \mathscr{C}\right)=\operatorname{Lstp}^{\mathrm{w}}(\bar{a}\urcorner \bar{b} / \mathscr{C}\right) .
$$

Since $\mathscr{A}$ is a-saturated, there is $\bar{a}^{*} \in \mathscr{A}$ realizing $\operatorname{Lstp}\left(\bar{a}^{\prime} / A \cup \bar{b}^{*}\right)$. By invariance, $\operatorname{Lstp}^{\mathrm{w}}\left(\bar{a}^{\prime} / \mathscr{C} \cup \bar{b}^{*}\right)$ is f-isolated over $A \cup \bar{b}^{*}$. We gain that

$$
\bar{a}^{*} \downarrow_{A \cup \bar{b}^{*}} \mathscr{C} \text {. }
$$

By stationarity, $\bar{a}^{*}$ realizes $\operatorname{Lstp}\left(\bar{a}^{\prime} / \mathscr{C} \cup \bar{b}^{*}\right)$. Furthermore by (4.1),

$$
\operatorname{Lstp}^{\mathrm{w}}\left(\bar{a}^{* \frown} \bar{b}^{*} / \mathscr{C}\right)=\operatorname{Lstp}^{\mathrm{w}}\left(\bar{a}^{\frown} \bar{b} / \mathscr{C}\right) .
$$

By Proposition $4.8(3), \bar{b}^{*}$ dominates $\bar{b}^{*} \cup \bar{a}^{*}$ over $\mathscr{C}$. Since $\bar{b}^{*} \downarrow_{E_{1}} \mathscr{B}$ by Lemma 4.11(1), using monotonicity, symmetry and dominance we get

$$
\bar{a}^{*} \cup \bar{b}^{*} \downarrow_{\mathscr{C}} \mathscr{B} .
$$

But now $\operatorname{Lstp}^{\mathrm{w}}(\bar{d} / \mathscr{C})$ is realized by $\bar{d}^{\prime} \subset A \cup \bar{a} \cup \bar{b}$, and $\bar{d} \downarrow_{\mathscr{C}} \mathscr{B}$ by monotonicity. By (4.3) and stationarity, $\operatorname{Lstp}^{\mathrm{w}}(\bar{d} / \mathscr{B})$ is realized in $A \cup \bar{a}^{*} \cup \bar{b}^{*}$ $\in \mathscr{A}$.

We recall the following well-known fact about Galois types.

LEMma 4.14 (Shelah). Let $(\mathbb{K}, \preccurlyeq \mathbb{K})$ be an AEC with amalgamation, joint embedding and arbitrarily large models.

(1) Let $\mathscr{A} \preccurlyeq \mathbb{K} \mathscr{B}, \mathscr{A} \preccurlyeq \mathbb{K} \mathscr{B}^{\prime},|\mathscr{A}|<\left|\mathscr{B}^{\prime}\right| \leq \kappa$ and $\mathscr{B}$ be $\kappa$-saturated. Then there is an automorphism $f \in \operatorname{Aut}(\mathfrak{M} / \mathscr{A})$ such that $f\left(\mathscr{B}^{\prime}\right) \preccurlyeq \mathbb{K} \mathscr{B}$.

(2) Two saturated models $\mathscr{B}_{1}, \mathscr{B}_{2}$ containing $\mathscr{A}$, such that $|\mathscr{A}|<\left|\mathscr{B}_{1}\right|=$ $\left|\mathscr{B}_{2}\right|$, are isomorphic over $\mathscr{A}$.

We can embed any countable model into an $\aleph_{0}$-saturated model. The previous lemma implies that when two models are saturated with respect to Galois types, they are isomorphic. 
THEOREM 4.15. Let $(\mathbb{K}, \preccurlyeq \mathbb{K})$ be a simple, tame finitary $A E C$ with the Tarski-Vaught property. Assume that $(\mathbb{K}, \preccurlyeq \mathbb{K})$ is a-categorical in some $\kappa>$ $\lambda \geq \max \left\{\mathrm{L}(\mathbb{K}), 2^{\aleph_{0}}\right\}$, where $\lambda^{\aleph_{0}}>\lambda$ and $\operatorname{cf}(\kappa)>\omega$. Then it is a-categorical in any $\kappa>\mathrm{L}(\mathbb{K})$.

Proof. By Theorem 3.34, ( $\mathbb{K}, \preccurlyeq \mathbb{K})$ is stable in $\lambda$. Then by Proposition 3.37 and Lemma 3.7, the class $(\mathbb{K}, \preccurlyeq \mathbb{K})$ is superstable. By Proposition 3.39, the only a-saturated model of size $\kappa$ is strongly saturated. Furthermore by Proposition 4.13, then any a-saturated model of size $>\mathrm{L}(\mathbb{K})$ is saturated with respect to weak Lascar strong types. Furthermore, by tameness and Theorem 3.20, any a-saturated model of size $>\mathrm{L}(\mathbb{K})$ is saturated with respect to Galois types. Then any two a-saturated models of the same size $>\mathrm{L}(\mathbb{K})$ are isomorphic.

Arguing as in Theorem 3.38, we can show the following corollary. The result is analogous to the Categoricity Conjecture of Shelah, except for the flaw that we assume the a-categoricity cardinal to have uncountable cofinality.

Corollary 4.16. Assume that $(\mathbb{K}, \preccurlyeq \mathbb{K})$ is a simple, tame finitary $A E C$ with the Tarski-Vaught property. If $(\mathbb{K}, \preccurlyeq \mathbb{K})$ is a-categorical in some $\kappa \geq \mathrm{H}$ with uncountable cofinality, then it is a-categorical in any $\kappa \geq \mathrm{H}$.

4.3. Questions. The first question is motivated by the fact that we would like to drop the assumption of uncountable cofinality in Corollary 4.16. We need this assumption in Proposition 3.37 to ensure that the acategorical model realizes all weak types over countable subsets.

QUESTION 4.17. Assume that $(\mathbb{K}, \preccurlyeq \mathbb{K})$ is a simple finitary class, a-categorical in some $\kappa>\mathrm{L}(\mathbb{K})($ or $\kappa \geq \mathrm{H})$ with countable cofinality. Does the unique a-saturated model of size $\kappa$ realize all weak types over countable subsets?

We believe that this question is related to the next question.

QUESTION 4.18. Assume that $(\mathbb{K}, \preccurlyeq \mathbb{K})$ is a simple finitary class, a-categorical in some $\kappa>\mathrm{L}(\mathbb{K})$ with countable cofinality. Is $(\mathbb{K}, \preccurlyeq \mathbb{K})$ weakly stable in $\kappa$ ?

One motivation for the study of finitary classes is to generalize the theory of (simple) excellent classes. We have also adapted many methods and concepts from excellent classes (see [13]). Since excellent classes are usually assumed to be $\aleph_{0}$-stable, this paper can be thought of as an attempt to generalize the study of excellent classes beyond $\aleph_{0}$-stability. We study the superstable case, but one could as well try to study the theory assuming only weak stability. In particular, can we prove a stability hierarchy theorem for 
weak types? Some preliminary results on the behaviour of independence in this case have been studied in Section 2.2.

\section{References}

[1] H. Adler, Explanation of independence, Ph.D. thesis, Albert-Ludwigs-Univ. Freiburg, 2005, arXiv:math/0511616v1.

[2] J. T. Baldwin, Categoricity, online book on nonelementary classes, http://www2.

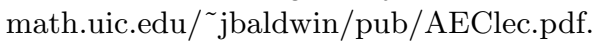

[3] J. T. Baldwin and A. Kolesnikov, Categoricity, amalgamation and tameness, to appear.

[4] R. Grossberg, Classification theory for abstract elementary classes, in: Logic and Algebra, Contemp. Math. 302, Amer. Math. Soc., Providence, RI, 2002, 165-204.

[5] R. Grossberg and A. Kolesnikov, Superior abstract elementary classes are tame, submitted.

[6] R. Grossberg and O. Lessmann, Abstract decomposition theorem and applications, in: A. Blass and Y. Zhang (eds.), Logic and its Applications, Contemp. Math. 380, Amer. Math. Soc., 2005, 231-259.

[7] R. Grossberg and M. VanDieren, Categoricity from one successor cardinal in tame abstract elementary classes, J. Math. Logic 6 (2006), 181-201,

[8] —, -, Galois-stability in tame abstract elementary classes, ibid., 25-49,

[9] - - - Shelah's Categoricity Conjecture from a successor for tame abstract elementary classes, J. Symbolic Logic 71 (2006), 553-568.

[10] T. Hyttinen, Uncountably categorical local tame abstract elementary classes with disjoint amalgamation, Arch. Math. Logic 45 (2006), 63-73.

[11] T. Hyttinen and M. Kesälä, Independence in finitary abstract elementary classes, Ann. Pure Appl. Logic 143 (2006), 103-138.

[12] - - - Categoricity transfer in simple finitary AEC, paper II in: Finitary Abstract Elementary Classes, Ph.D. thesis, Dept. Math. Statist., Univ. of Helsinki, 2006.

[13] T. Hyttinen and O. Lessmann, Simplicity and uncountable categoricity in excellent classes, Ann. Pure Appl. Logic 139 (2006), 110-137,

[14] T. Hyttinen and S. Shelah, Strong splitting in stable homogeneous models, ibid. 103 (2000), 201-228.

[15] D. W. Kueker, Finitary abstract elementary classes and $L_{\infty \omega}$, draft, 2007.

[16] O. Lessmann, An introduction to excellent classes, in: A. Blass and Y. Zhang (eds.), Logic and its Applications, Contemp. Math. 380, Amer. Math. Soc., 2005, 231-259.

[17] - Upward categoricity from a successor cardinal for tame abstract classes with amalgamation, J. Symbolic Logic 70 (2005), 639-660.

[18] S. Shelah, Finite diagrams stable in power, Ann. Math. Logic 2 (1970), 293-300.

[19] - Classification Theory and the Number of Nonisomorphic Models, North-Holland, 1978; 2nd rev. ed., 1990.

[20] - Classification theory for for nonelementary classes, I. The number of uncountable models of $\psi \in L_{\omega_{1}, \omega}$. Parts A and B, Israel J. Math 46 (1983), 212-273.

[21] - Classification of non elementary classes II. Abstract elementary classes, in: J. T. Baldwin (ed.), Classification Theory (Chicago, 1985), Lecture Notes in Math. 1292, Springer, Berlin, 1987, 419-497.

[22] - Categoricity of abstract classes with amalgamation, Ann. Pure Appl. Logic 98 (1999), 261-294.

[23] —, Categoricity in abstract elementary classes: going up inductive step. 
[24] S. Shelah, Toward classification theory of good $\lambda$ frames and abstract elementary classes, arXiv:math.LO/0404272, 2004.

[25] S. Shelah and A. Villaveces, Toward categoricity for classes with no maximal models, Ann. Pure Appl. Logic 97 (1999), 1-25.

Department of Mathematics and Statistics

University of Helsinki

P.O. Box 68

FI-00014 Helsinki, Finland

E-mail: tapani.hyttinen@helsinki.fi

meeri.kesala@helsinki.fi

Received 25 August 2006;

in revised form 10 July 2007 